\title{
Discourses of Vulnerability: Kiribati, I-Kiribati Women and Forced Migration Due to Climate Change
}

\author{
By \\ Holly Alison Haultain Kings Mansfield
}

\author{
A Thesis \\ Submitted to Victoria University of Wellington \\ in fulfilment of the requirements for the degree of \\ Master of Arts \\ in Pacific Studies
}

Victoria University of Wellington 


\begin{abstract}
Discourses of vulnerability abound in climate change literature; both particular types of places and particular groups of people are routinely considered especially vulnerable to the effects of climate change. Such discourses are employed by the international community in a technical manner-attempting to categorise current and expected degrees of impact on places and populations-and also to support the advocacy of urgent action to respond to climate change.

This thesis examines the manner in which discourses of vulnerability are being invoked in discussions about the impacts of climate change and considers what impact this has on the people and places that are being described in this way. This research focuses specifically on Kiribati and I-Kiribati women and explores, in particular, how the debate about forced migration (as a possible outcome of climate change) has been shaped by vulnerability discourses. Both Kiribati, as a specific type of geographical entity, and women, as a category of people, have been described as having extreme vulnerability to climate change. It is not new for either Pacific Islands or women to be framed as "vulnerable"; however with the increased attention to climate change, vulnerability discourses are being used with such frequency that it is virtually impossible to find literature on the Pacific and women that does not reference their vulnerability. The use of such an emotive term raises questions. Who is naming and claiming vulnerability? What impact does such language have on those that are portrayed in this way? And, what are the long-term consequences of such terminology being used? Interesting questions are also raised regarding discursive similarities between presentations of vulnerable women and vulnerable islands.

This thesis addresses these questions by analysing international literature on climate change and forced migration-especially that produced by the Intergovernmental Panel on Climate Change (IPCC)—between 1990 and 2007, and contrasting this with close analysis of public discourse from two vocal I-Kiribati advocates, Pelenise Alofa and Maria Tiimon, from 2009 to 2011. Conclusions are drawn from this analysis regarding the power relationships embedded in discourse, and the possible ramifications of language use for the construction of policy.
\end{abstract}




\section{Acknowledgements}

This journey of writing a thesis has been one with many ups and downs and it is the love and support of a great many people that has enabled me to complete it. I would like to take this opportunity to thank those people.

I would like to start by thanking the people of Kiribati who, since climate change has become such a pressing issue for the international community, have been inundated with researchers, scientists, and volunteers, all working on defining the issues that are perceived to be affecting Kiribati, formulating solutions, and researching both the place and its people. I am very much aware that this increased attention has been both beneficial and at times unwanted. I therefore want to thank the people of Kiribati for, although they are not aware of it, being subject to yet another research project. I hope that I have conducted this research with an awareness of your voice, concerns and agency within the climate change context.

Secondly I would like to thank both Pelenise Alofa and Maria Tiimon who, through the use of their various speeches, presentations and activism have brought this thesis to life and provided the content that I have been able to use in my analysis. The way that these women deliver their speeches, the language that they use and the passion with which they speak, has brought a very real and rich sense of the stakes of climate change for Kiribati, and for them as I-Kiribati women, to the debates.

I also want to extend thanks to my family and friends who have consistently supported me, pushed me along, dedicated babysitting hours, to both me and my son, and excused my absence from their lives while this thesis has been put together. I cannot say thank you enough.

I would like to extend a special thank you to my other mother Mandy McDonald, Mandy you have been the greatest support to me throughout this whole process. Your dedication of time, your constant encouragement, support, understanding, and, when called for, firm hand has enabled me to complete this thesis. It is true that without you I am not sure that I could have done this. So thank you Mandy and I love you. 
Thanks also needs to be given to my son, Harvey Warner, who has provided all the light relief that I have needed and constantly reminds me that life is full of wonder and it's important to take a break and a deep breath and appreciate it.

This thesis would also not have been completed without the enormous amount of time that my supervisor, Dr. April Henderson, has given to both me and this research. April, you have guided me through a very fulfilling and at times challenging process with your calmness and positivity. Your wealth of scholarly knowledge which you have shared with me has been inspiring. Thank you for knowing exactly when to push me, encourage me, hurry me and ground me. I feel very fortunate to have had the pleasure of you as my supervisor.

Lastly I would like to thank the people that are no longer with me, Luci Highfield and Mark (Bolt) Haultain. Your strength, courage and determination in your ultimate battles have provided me with great examples which I have learnt so much from. I am only sorry that I cannot share the excitement of finishing this thesis with you.

I would like to finish with a poem for all those people that have helped me along the way, both in their examples and with their presence.

\section{Arohanui}

Big love, that's what it means.

Aroha Nunui means huge love.

Aroha Nunui Rawa means very huge love.

Aroha Nunui Rawa Ake means bigger very huge love.

Aroha Nunui Rawa Ake Tonu means bigger enduring very huge love. Aroha Nunui Rawa Ake Tonu Atu means biggest enduring hugest love, Which are some of the lengths and times of our longing

(Robert Sullivan) 


\section{TABLE OF CONTENTS}

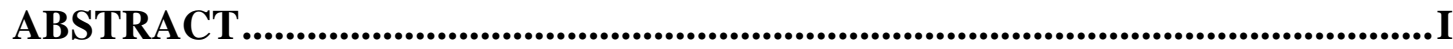

ACKNOWLEDGEMENTS …….................................................................... II

LIST OF ABBREVIATIONS ...................................................................... VI

LIST OF TABLES ..........................................................................................

CHAPTER ONE: INTRODUCTION........................................................... 1

CHAPTER TWO: IMAGES OF VULNERABILITY WITHIN THE CLIMATE CHANGE DEBATE: PACIFIC ISLANDS AND WOMEN - A REVIEW OF

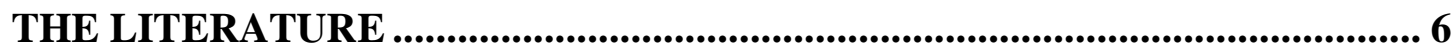

The Production of Climate Change KNOWLEdGE …………………............... 7

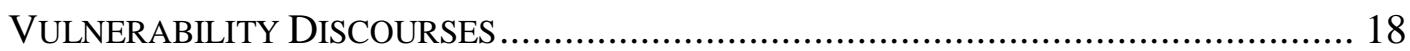

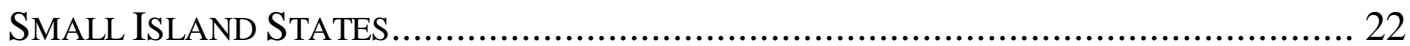

FoRCED MigRATION AS A CONSEQUENCE OF CLIMATE CHANGE .............................. 29

WOMEN AND VULNERABILITY ........................................................................ 33

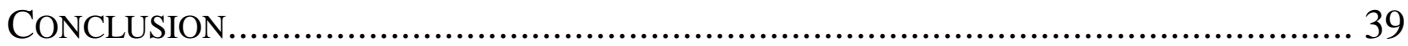

CHAPTER THREE: OUTLINE OF METHODOLOGICAL APPROACH....... 41

WHY QUALITATIVE RESEARCH? ...................................................................... 41

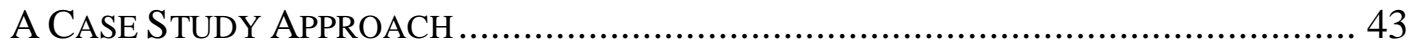

CRITICAL DISCOURSE ANALYSIS................................................................... 46

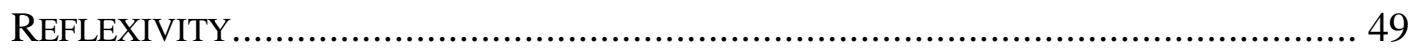

CHAPTER FOUR: ARTICULATIONS OF ISLANDS, WOMEN AND

FORCED MIGRATION FROM OUTSIDE THE PACIFIC .................................. 54

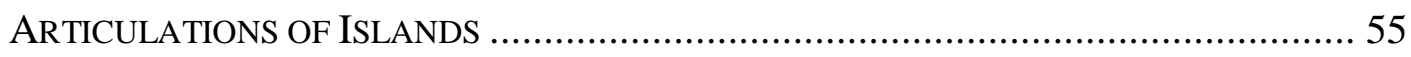

ARTICULATIONS OF WOMEN ........................................................................... 59

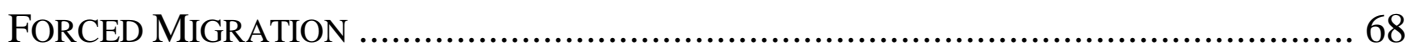

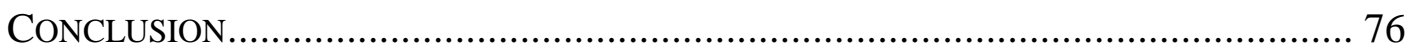

CHAPTER FIVE: ARTICULATIONS OF KIRIBATI'S AND I-KIRIBATI WOMEN'S VULNERABILITY BY PELENISE ALOFA AND MARIA TIIMON

FRAMING THE PACIFIC: RECAP OF THE THEORETICAL DEBATE................................. 80

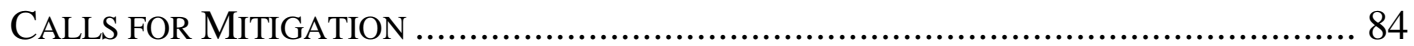

THE APPLICATION OF BIOPHYSICAL VULNERABILITY FRAMEWORKS ........................ 85

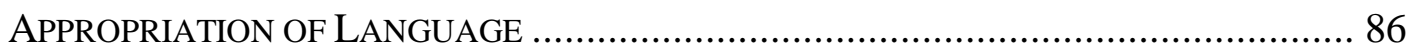

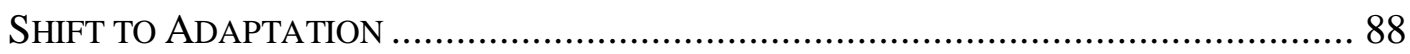

FRAMING OF SOCIAL VULNERABILITY BY ALOFA AND TIIMON.................................. 89

Alofa AND TIIMON REFRAME THE DeBATE ............................................................ 91

ARTICULATIONS OF WOMEN BY ALOFA AND TiIMON: VULNERABLE OR AGENTS OF

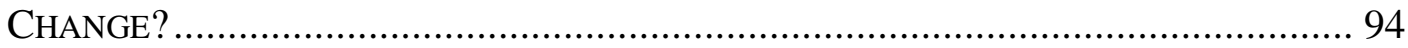

THEORETICAL EXPLANATIONS OF ALOFA's AND TIIMON'S APPARENT LIMITED

ATTENTION TO I-KIRIBATI WOMEN.................................................................... 97

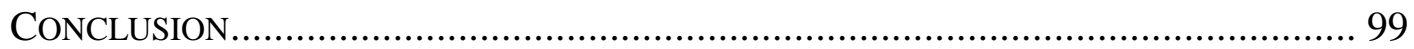

CHAPTER SIX: ARTICULATIONS OF FORCED MIGRATION BY

PELENISE ALOFA AND MARIA TIIMON..................................................101

CAUSES OF ForCED MigRATION ARTICULATED By ALOFA AND TIIMON .................102

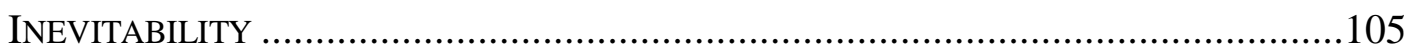

IMPACT OF FORCED MIGRATION ……………………………….......................110 
How to MigRate IF ForCED to Migrate .................................................114

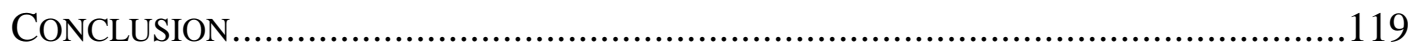

CHAPTER SEVEN: CONCLUSION ..........................................................121

REVIEWING THE RESEARCH AIMS, MOTIVATION AND APPROACH ..........................121

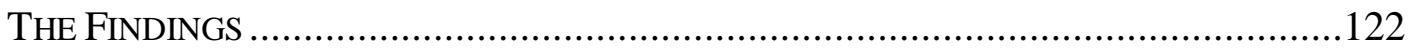

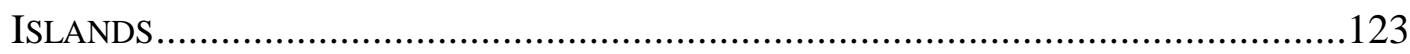

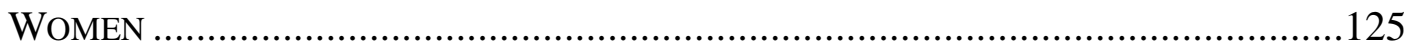

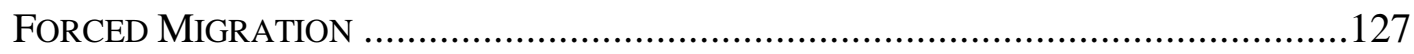

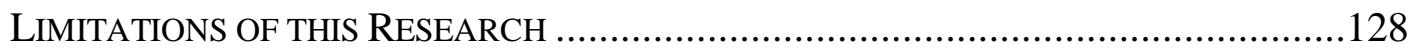

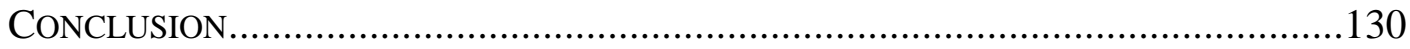

BIBLIOGRAPHY ...........................................................................................133 


\section{List of Abbreviations}

ASPEI

CAN

CAPWIP

CC

CDA

COP

ESSP

IPCC

LDCs

NGOs

SIDS

SPREP

SRHR

UNESCO

UNFCCC

WMO
Association of South Pacific Environmental Institutions

Climate Action Network

Center for Asia Pacific Women In Politics

Climate Change

Critical Discourse Analysis

Conference of the Parties (UNFCCC)

Earth System Science Partnership

Intergovernmental Panel on Climate Change

Least Developed Countries

Non-Governmental Organisations

Small Island Developing States

Secretariat of the Pacific Regional Environment Programme

Sexual and Reproductive Health and Rights

United Nations Education, Scientific and Cultural Organisation

United Nations Framework Convention on Climate Change

World Meteorological Organisation 


\section{List of Tables}

Table 1: Intrinsic Vulnerability in Small Island Developing States

Table 2: Dualisms in Climate Change

Table 3: Dualisms Based on Sex 


\section{Chapter One: Introduction}

Discourses of vulnerability abound in current climate change literature. Small, lowlying, developing, sinking, tiny, remote, isolated and vulnerable are terms routinely used to describe Kiribati and other Pacific atoll nations. Reference to Kiribati's vulnerability has become such an accepted practice that it is almost impossible to find climate change-related discussions of Kiribati in which terms suggestive of vulnerability do not feature. Even those who offer alternative adjectives to describe Kiribati-vibrant, resilient, beautiful, sustainable, resource- and environmentally rich, connected-make what, on the surface, appear to be similar references to the country's vulnerability in the face of climate change.

Women, too, are cast as particularly vulnerable in climate change literature. Sub-sets of this literature point to special qualities or conditions-poor, ignored, unskilled, left behind - that render women a special category of concern. However, alternative renderings of women as close to nature, specially skilled, leading the way, and agents of change are also evident in climate change debates. While appearing to reject discourses of vulnerability, these alternative depictions similarly presume that women have special qualities and conditions of being (whether innate or socially constructed) that warrant attention.

How do these various sets of sometimes opposed, sometimes congruous terms coexist in climate change debate? Who employs them and for what purpose? Are the terms used to mean different things, for different reasons, by different commentators? How are they used by those presumed to be 'outsiders', in geographical and gender terms, and how do they feature in the language of the 'insiders' who are implicated as I-Kiribati and, specifically, I-Kiribati women? Have historical and current power relationships between the Pacific and the non-Pacific, and men and women, influenced the choice of language? And, finally, what are the potential impacts of language use in shaping understanding of the impacts of climate change, and in developing strategies for addressing them?

This thesis addresses these questions by analysing international literature on climate change and forced migration-especially that produced by the Intergovernmental Panel on Climate Change (IPCC) — between 1990 and 2007, and contrasting this with close analysis of public discourse from two vocal I-Kiribati 
advocates, Pelenise Alofa and Maria Tiimon, from 2009 to 2011. Conclusions are drawn from this analysis regarding the power relationships embedded in discourse, and the ramifications of language use for the construction of policy. These questions will be explored and examined against the backdrop of discussions of forced migration. The meanings that are attached to both women, as a special category of people, and Kiribati, as a special type of place, will be examined using both Pacific and feminist theory. This research presents the argument that the language that is being used plays a significant role in influencing outcomes for Kiribati and I-Kiribati women.

Chapter two provides a review of the literature, introducing key texts, relevant theoretical concepts and frameworks, and begins to identify the ways in which vulnerability discourses are appearing in the climate change debate. This chapter is separated into five sections. The first section raises questions regarding the production of knowledge on climate change and introduces a theoretical analysis of how climate change knowledge is generated, who generates this knowledge, and whose knowledge counts in the climate change literature. Attentive to pragmatic considerations regarding scope, this section also outlines the decision to focus, in particular, on the Intergovernmental Panel on Climate Change (IPCC) as an international organisation that has considerable influence on the validity of climate change evidence, how climate change is articulated and understood, and-through the dissemination of this knowledge-significant influence over policy decisions related to climate change. The second section introduces key understandings and definitions of vulnerability that are drawn upon throughout the climate change literature and this research. This chapter then goes on to illustrate the relevant arguments, theories and concepts that have been applied to Pacific Islands, women and forced migration as topics of discussion within climate change literature. The third section specifically examines the literature on Pacific Islands, drawing on the debates and counter debates about the Pacific's vulnerability. This section considers how the Pacific is framed within an international context as well as critical challenges to these framings. Section four looks at the literature on forced migration, specifically with regard to Kiribati; this section demonstrates the consistency of references to the reality of forced migration for places such as Kiribati. And finally section five provides an overview of the relevant arguments about why and how women should and need to be included within 
the climate change debates. These sections demonstrate the multiple and often contrasting concepts that are present throughout the rest of this research.

Chapter three outlines the approach that this research takes to answer questions regarding how vulnerability discourse is being applied in debates about climate change, and what impact this has on the people and places discussed. As this could potentially become an enormous task, the justifications for narrowing this research to focus on Kiribati and I-Kiribati women using elements of a case study approach are explained. It was felt that research methodologies that enable the meaning of language to be explored within a broader context were imperative to answering the stipulated questions. This section therefore outlines why it was felt that a qualitative approach, coupled with critical discourse analysis, would allow for a greater depth in understanding who is using vulnerability discourse, what they mean when it's used, and what impact this may have on discussions of climate change. When utilising qualitative approaches and conducting critical discourse analysis in research, the significance and emphasis of meaning is influenced by the theoretical standpoint that is being taken to analyse the meanings that are uncovered. The methodology chapter allows for the theoretical underpinning of analysis to be made explicit. As this research is specifically looking at how Kiribati and I-Kiribati women are being talked about, it was felt that a combination of Pacific studies theory, Pacific feminist theory, and feminist theory was the most appropriate theoretical underpinning to analyse vulnerability discourse. As with all research projects, providing a transparent approach and analysis allows for greater robustness of the research, therefore it was felt that providing a section on reflexivity would allow for situational perspectives that may influence this research to be addressed.

Chapters four, five and six make up the main body of analysis where the concepts and questions introduced here and in the literature review are explored via the specific case of Kiribati. These chapters examine how vulnerability discourses have been applied: they interrogate the understandings and meanings of terms applied to Kiribati and I-Kiribati women, explore whether the identity of the person using vulnerability discourses alters the meanings attached, and, lastly, questions the impact of these varying articulations of vulnerability on the way that forced migration is articulated.

Chapter four provides a detailed analysis of the way that the international community is using vulnerability discourse. It introduces concepts of grounding adjectives that seek to anchor vulnerability discourse within broader vulnerability 
frameworks. This chapter is presented in sections. The first looks at constructions of Pacific Islands broadly and Kiribati specifically. The second section focuses on IKiribati women and how concepts of vulnerability or agency are being applied, the relevance of such categorisation and the impacts of classifying women as either. The third section draws together the articulations of Kiribati and I-Kiribati women and examines how the language that is being drawn upon impacts on concepts of forced migration. Using Pacific and feminist theoretical standpoints which were introduced in Chapter two, this chapter interrogates the way vulnerability has been constructed and applied to Kiribati and I-Kiribati women and how this then impacts upon conceptions of Kiribati and I-Kiribati women. It then examines how this influences the way forced migration is spoken about. This chapter makes the case that the way in which Kiribati is being framed has a long historical tradition-one in which vulnerability is assumed as an innate quality of islands, and that climate change, rather than being the cause of vulnerability, is in fact merely highlighting and exacerbating the vulnerability of Kiribati. The concepts that were introduced in Chapter one regarding how women have been articulated within the climate change debate, and why they are articulated in this way, are then applied to I-Kiribati women and examined. Questions are asked about whether the broader framings of women within the climate change debate can be applied to I-Kiribati women and what this tells us about the construction of vulnerability in relation to I-Kiribati women. Lastly, this chapter uses the analysis of Kiribati and I-Kiribati women's vulnerability and investigates how this can be seen to have shaped the current conversations on forced migration. This chapter mounts the argument that the viewing of both Kiribati and IKiribati women as innately vulnerable places limits on the way that other adaptation strategies are thought about and discussed.

Chapter five introduces Pelenise Alofa and Maria Tiimon, two I-Kiribati women who have become well-known spokespeople for Kiribati and climate change issues. These vocal and prolific advocates for Kiribati have delivered numerous speeches at international forums as well as local events, conducted frequent media interviews, and been used as the voices and faces of several campaigns focused on climate change. This chapter examines the way that these women are expressing both Kiribati and IKiribati women's vulnerability. It analyses the language that they use, the way that they use it, and the impact that their language use has. Conclusions are drawn about 
the similarities and differences in the way they express vulnerability, describe Kiribati and talk about I-Kiribati women.

Following on from Chapter five, Chapter six hones in on the specific issues of forced migration. It draws together the ways that Alofa and Tiimon have described Kiribati and I-Kiribati women and examines if and how this has altered the way that forced migration is being talked about by them. This chapter discusses notions of inevitability, the ways the impacts of climate change have been described, and also the predicted impact of forced migration on I-Kiribati. This chapter thus allows for Alofa's and Tiimon's discussions of forced migration to be compared and contrasted both with each other and with the articulations by the international community presented in Chapter three. Conclusions are drawn regarding the significance of the ways that Kiribati is framed, and how this affects discussions of forced migration.

Lastly, Chapter seven offers some final consideration of the different theoretical concepts and articulations of vulnerability, Kiribati, I-Kiribati women and forced migration discussed throughout the thesis. It is within this chapter that all the at-times contradictory notions of both Kiribati and I-Kiribati women are drawn together and the findings of this research are set out. This concluding chapter also notes some of the limitations and gaps of this research project, acknowledging and suggesting salient avenues for further research that fall outside the scope of this thesis. 


\section{Chapter Two: Images of Vulnerability within the Climate Change Debate: Pacific Islands and Women - A Review of the Literature}

The topic of climate change has generated a substantial amount of interest from the international community since the early 1980s. Vast bodies of research have been produced on the science of climate change, the impacts of climate change, and the possible options for combating climate change or adapting to a world where climate change is a reality. Contributing to the literature on climate change are a wide range of scientists, social scientists, economists, politicians, activists, special interest groups and numerous others. Throughout this expansive breadth of literature, references to vulnerability are routinely invoked when considering the impacts of climate change on Pacific Islands. There is also a subset of literature that focuses on the vulnerability of women and the importance of having women included in climate change debates and policies. Due to vulnerability becoming synonymous with the Pacific Islands region and women in general in the climate change debate, it is important to explore how and why discourses of vulnerability are being invoked and what impact these may have on shaping both the debate and policy decisions.

This chapter will provide an overview of relevant literature on climate change and vulnerability, and offer an initial exploration and interrogation of that literature through critical frames provided by Pacific studies and feminist theory. I will begin by exploring literature on the production of climate knowledge. I ask, who produces knowledge around climate change and what are the ramifications of imbalances between people's abilities to produce and circulate their understandings of climate change? I will then provide a brief overview of the literature outlining the definitions and models of vulnerability discourses within the climate change debate and what impact this has on the debate. The chapter will subsequently look at the current literature that examines the impacts of climate change on small island states, focusing specifically on Kiribati as a case example. Following this, I will look at forced migration as a consequence of climate change. Lastly, I will examine discourses about women within the climate change debates with particular reference to literature on forced migration. Throughout this literature review I will be using both Pacific studies and feminist analyses to interrogate the use of vulnerability as a descriptive 
term for Pacific Islands such as Kiribati, and for I-Kiribati women, within the climate change debate.

\section{The Production of Climate Change Knowledge}

The development of knowledge about anthropogenic climate change has undergone significant shifts since it was first proposed as a viable scientific explanation for the speeding up of the natural changes that occur within the earth's climate. Since the 1980s, the issue of climate change has increasingly become one that is not restricted to the scientific community; policy makers, activists, and popular media have all participated in the climate change debate. It has become a popular issue of widespread interest. The production of knowledge about anthropogenic climate change is an important element to examine as the knowledge that is being produced has a direct impact on the strategies that are being explored for dealing with a changing climate. There is already a broad body of literature generated by debates over whether there is a human-induced element to our changing climate. While recognising the importance of this material, this literature review does not intend to rehearse the various positions in these debates and is instead interested in meta questions about the production of knowledge about climate change: specifically, who produces this knowledge and why do these contexts of knowledge production matter?

A pivotal place to start when looking at the accumulation of knowledge on climate change is the Intergovernmental Panel on Climate Change (IPCC). Initially, knowledge about climate change was largely restricted to the scientific community. As Daniel Bodansky, a specialist in international environmental law, explains, "The development of the climate change issue initially took place in the scientific arena as understanding about the greenhouse problem improved" (Bodansky 2001: 89). Andrew Dessler, a Professor of Atmospheric Sciences, and Edward Parson, a Professor of Law, reference the time it took to translate scientific concern into governmental interest and action.

Like many serious environmental issues, global climate change attracted virtually no public or political attention in the 1960s, and only a little during the energy-policy debates of the 1970s. By the early 1980s, as it became increasingly clear that warming from greenhouse gases was a serious concern, scientists and scientific organisations began trying to persuade governments to pay attention to 
the climate problem. They had little success until 1988 (Dessler \& Parson 2010: 22).

As the scientific knowledge about climate change developed it became widely recognised that there was a need for a governmental response. Paterson argues that,

During the period 1985-8, many more scientists also became convinced of the need, not only for some framework convention analogous to that developed for ozone depletion (the Vienna convention), but for strong preventative action on global warming (Patterson 1996: 30).

As an example, Patterson highlights a 1988 statement made by Kenneth Hare, who was chairman of the Climate Programme Board of Canada, and also chair of the Advisory Group on Greenhouse Gases.

Until a short while ago, my own position was...that the evidence was too equivocal to do more than give a yellow alert to governments...I was an announced wait-and-see conservative...I can and do tell them [government] that they should base their environmental planning on the assumption that the greenhouse warming will continue and accelerate (Paterson 1996: 30).

This illustrates the constantly evolving body of knowledge being generated by scientific communities and the increased awareness that climate change was no longer a scientific question but was instead becoming recognised as a problem that required governmental policy solutions.

The increased awareness from the scientific community that climate change was a real threat to the world, and that governments needed to start putting international policies into place to mitigate the possible impacts of climate change, is a possible impetus for the creation of the IPCC. Many have credited the science workshops held in Villiach (Austria) during the 1980s as having galvanised political action on climate change. Sir John Houghton, Chairman of the Scientific Assessment Working Group of the Intergovernmental Panel on Climate Change 1988- 2002, asserts that, "The report of a scientific meeting held in Villiach, Austria in 1985 ...began to alert governments and the public at large to the potential seriousness of the issue..." (Houghton 2002: 1). Paterson states, “The Villiach Conference made recommendations which emphasised the need for economic, social and technological research into policy options for responding to any potential climate change” (Paterson 
1996: 31). Support for this view of the seminal role of the conference is shown by a World Meteorological Organisation (WMO) statement made after the conference.

Support for the analysis of policy and economic options should be increased by governments and funding agencies. In these assessments the widest possible range of social responses aimed at preventing or adapting to climate change should be identified, analysed, and evaluated (World Meteorological Organisation 1986: 3).

Jon Barnett, John Campbell and Michael Paterson argue that it was through these meetings that the IPCC was established, with the support of the scientific community and the US Government. Barnett and Campbell claim, "They [scientists] also suggested that there be a formal institution for international scientific collaboration to enhance understanding of the causes and impacts of, and solutions to, climate change” (Barnett \& Campbell 2010: 52). Physicist Spencer Weart, however, offers the contrasting opinion that the creation of the IPCC was in response to critics who argued that independent scientific groups, who had until then been bringing this issue into the political arena, were gaining control of the debate (Weart 2003). Weart argues, "If the process continued in the same fashion, the sceptics warned, future groups might make radical environmentalist pronouncements. Better to form a new system under the control of government representatives” (Weart 2003: 158). This pressure, Weart claims, prompted the WMO and other United Nations environmental agencies to create the IPCC (Weart 2003).

The IPCC's role is not to create knowledge but rather to act as an independent body that assesses the scientific knowledge that is being produced and offers policy advice to policy makers. As Barnett and Campbell indicate, "The IPCC was established in 1988 to provide independent scientific advice on the issue of climate change” (Barnett \& Campbell 2010: 58). They go on to explain,

It is important to recognise that unlike the ESSP [Earth System Science Partnership] programmes, the IPCC does not conduct any original research ... The IPCC's work is limited to assessing the latest literature on climate change, its impacts, and options for adaptation and mitigation (Barnett \& Campbell 2010: 59-60).

Although the IPCC does not produce original research, its role in the production of knowledge is significant as it provides legitimacy to the scientific research that is being produced. The IPCC also highlights where there are specific gaps in the 
research and makes recommendations for further scientific research into climate change. This position that the IPCC holds means that it wields significant influence on what knowledge counts within the climate change debates. The IPCC acts as legitimiser of the orthodox scientific opinion. This scientific knowledge then influences government policies on climate change.

Since the IPCC's creation it has become instrumental in the dissemination of scientific knowledge, which is constantly evolving, and provision of advice on the best policy practices for adaptation and mitigation for governments around the world to follow. Unlike earlier working groups looking at climate change, the IPCC was not restricted to input from the science field alone; it was instead made up of a mix of both scientists and international government policy makers. As Weart describes, "Unlike all the earlier national academy panels, conferences, and other bodies, the IPCC brought together people who spoke not just as science experts, but as official representatives of their respective governments” (Weart 2003: 158).

This intergovernmental organisation has had a direct influence on policies to mitigate climate change. Barnett and Campbell highlight the influence that the IPCC has on international agreements, “The First Assessment Report of the IPCC informed the construction of the United Nations Framework Convention on Climate Change (UNFCCC). The Second Assessment Report (1995) was also used to inform the negotiating positions of some parties in the lead up to the Kyoto Protocol” (Barnett \& Campbell 2010: 59). Through these reports an evolving body of knowledge has been produced.

Due to the evident influence that the IPCC has on policy, it is important to interrogate who is producing the research that the IPCC reports, and examine how it shapes our knowledge on climate change. The IPCC in its mandate asserts,

Today the IPCC's role is also, as defined in Principles Governing IPCC work,...to assess on a comprehensive, objective, open and transparent basis the scientific, technical and socio-economic information relevant to understanding the scientific basis of risk of human-induced climate change, its potential impacts and options for adaptation and mitigation. IPCC reports should be neutral with respect to policy, although they may need to deal objectively with scientific, technical and socioeconomic factors relevant to the application of 
particular policies (Intergovernmental Panel on Climate Change 2011:

1).

As has been shown the scientific knowledge that is being disseminated through the IPCC has a direct impact on policy responses to climate change.

The IPCC claims to be an institution that is objective in its analysis of the scientific research that is being produced. Assessing the nature of this purported objectivity is an important element of the climate change debate when looking at how knowledge is produced, who produces it, and for what purpose it is produced. Much academic literature is devoted to debating the merits and, in fact, achievability of objectivity. Many social scientists, including feminists, have been heavily critical of the idea of objectivity (Westmarland 2001, Maynard 1994, Ramazanoglu \& Holland 2002). As Ramazanoglu and Holland state, “To be objective the researcher’s findings must be impartial, general and free from personal and political biases” (Ramazanoglu \& Holland 2002: 48). Critics such as Donna Haraway, a feminist science studies scholar, contend that absolute objectivity is not achievable, instead arguing that, "Science has been about a search for translation, convertibility, mobility of meanings, and universality—which I call reductionism, when one language (guess whose) must be enforced as the standard for all the translations and conversions... there is finally only one equation” (Haraway 1991: 187-188). Haraway points to the ever present power dynamics embedded in scientific discourse to argue that scientific knowledge is in fact subjective, influenced by the dominant positions of knowledge i.e. white male knowledge.

Peter Haas, a political scientist whose main research area is the role of science in international and environmental regimes, also questions that notion of scientific objectivity. Haas takes the position that,

Science is politically tainted and suspect. Organised modern science embodies implicit values and control, so that decisions made with scientific warrants may unconsciously reflect such hidden values. In addition the distributional consequences of science-based advice are themselves political. Science is political in its consequences, because some benefit and others suffer as a consequence of policy options that are supported by the application of scientific understanding... (Haas 2004: 571). 
Haas's argument highlights the political nature of scientific knowledge with reference in particular to the policy advice that arises from science-based knowledge. This is a particularly salient point for climate change policy as it is informed from scientific advice and much of the public discourse rests on what constitutes the "objective" truth.

Political scientist Mary Pettenger highlights the influence that scientific knowledge has on policy whilst also claiming that the same can be said in reverse. Pettenger says,

At a minimum, scientific knowledge constitutes a necessary (albeit by no means sufficient) condition for policy advancement, shaping political discussions and outcomes as much as these shape competing framings of scientific knowledge (Pettenger 2007: 176).

This statement, arguing that scientific knowledge is shaped by political influence, calls into question the idea of a truly objective form of scientific knowledge. Drawing on the analyses of French philosopher Michel Foucault, Gary Gutting also elaborates on relationships between power, knowledge, and legitimacy,

Particular investigations were structured by which concepts and statements were intelligible together, and how those statements were organised thematically, which of those statements counted as 'serious', who was authorized to speak seriously, and what questions and procedures were relevant to assess the credibility of those statements that were taken seriously (Gutting 2005: 96).

These arguments regarding objectivity are important to take into account when examining issues of power and knowledge. For those who strive for objectivity, the need to question who is involved in creating knowledge about climate change is unnecessary. However for those critical of the premise that objectivity can be achieved, the question of who produces knowledge, and the power that it carries, is an extremely important one to examine. As Haraway states, "We do need an earth-wide network of connections, including the ability partially to translate knowledges among very different—and power-differentiated—communities” (Haraway 1991: 187).

Critics of the IPCC have pointed to the lack of representation of developing countries' governments and scientists, and argue that through this lack of representation, the objectivity of the scientific knowledge being produced is compromised. Kandlikar and Sagar for example put forward that, "Globally, the 
majority of the climate analysts are from the industrialized countries...” (Kandlikar \& Sagar 1999: 120). This is evident when looking at the break down of the scientists involved in the reports of the IPCC. Barnett and Campbell identify that, "Like the ESSP programmes, the majority of researchers participating in the IPCC reports are from developed countries” (Barnett \& Campbell 2010: 61). Barnett and Campbell go on to highlight this by showing the specific numbers of contributors to published reports of the IPCC,

Of the 393 convening lead authors, lead authors and review editors that participated in the Fourth Assessment Report (across all working groups), two thirds came from the OECD countries (this does not include the 16 researchers from Russia), and one third came from outside the OECD. Of this latter group only 19 (3 percent of all authors) came from Small Island Developing States, of which only two (0.3 per cent of all authors) came from the Pacific Islands (Barnett \& Campbell 2010: 61-2).

This lack of representation amongst the creators of scientific knowledge has a direct impact on the focus of climate change research and the potential outcomes of policies related to the scientific knowledge. Milind Kandlikar and Ambuj Sagar highlight the influence of cultural perspectives in science and argue that through the lack of inclusion of a wide array of cultural positions, the views that are being represented as universal are in fact biased in favour of a northern hemisphere perspective.

Studies of cross-national and cross cultural perspectives have shown that scientific problem definitions and paradigms can be framed in ways that reflect underlying cultural presuppositions...Consequently many of the 'global' analyses are, more often than not, strongly coloured by a northern perspective... (Kandlikar \& Sagar 1999: 120).

These commentators highlight both the lack of representation within the IPCC and the possible impact that this lack of representation has on the scientific knowledge emanating from the IPCC. Kandlikar and Sagar's argument touches on the position that there are different ways of knowing, or as Donna Haraway has summarised, “situated knowledge." (Haraway 1988) These positions lead to the conclusion that the lack of Pacific scientific voice within the IPCC will impact on how the international community shapes the 'problem' for the Pacific and, in a related vein, on how climate change issues are dealt with by Pacific countries. 
Many Pacific theorists have been active in promoting the notion that the Pacific has its own ways of knowing and that acknowledgment and inclusion of Pacific knowledge is vital for understanding issues affecting Pacific Islanders. Pacific theorists have critiqued the long history of exclusion by outsiders of Pacific ways of knowing and the subsequent impact that this has had on policies. Pacific-based scholars Elise Huffer and Ropate Qalo claim that, "Ignorance or dismissal of Pacific thought prevails in academia, which in turn has impacted on policymaking in Pacific countries. This situation is in part the result of disciplinary biases and omissions" (Huffer \& Qalo, 2004: 87).

This argument is supported by Melissa Finucane, who specifically examines the topic of climate change and the inclusion of Pacific knowledge, and argues, "The people of the Pacific hold diverse beliefs about climate change and these beliefs inform their decisions. In addition, a dynamic social context influences the extent to which people are able to respond meaningfully to climate impacts" (Finucane 2009: 1). Academics and activists arguing for a more inclusive environment for the generation of climate change research argue that, without inclusion and understanding, solutions from within Pacific island countries will not take shape. Finucane underscores this point, emphasising that,

To solve the problem of the climate crisis, policymakers need to set a risk management agenda that integrates sound social science with an understanding of how that science is interpreted and translated into action in society. They will need to work not only with scientists, but also with cultural leaders, theologians, philosophers, and community groups (Finucane 2009: 1).

Finucane is highlighting a link between the importance of drawing knowledge from the international scientific community and then interpreting it within specific cultural understandings.

Senior research scientist Paul Runci, like Finucane, notes the importance of inclusion of developing countries' voices; however, Runci notes that this inclusion will not only mean that scientific knowledge is better understood and implemented but it will also allow for developing countries to present their specific concerns to the international stage. Runci argues,

To the extent that scientists from developing countries are able to augment their participation in the generation of new scenarios, they 
will contribute both to capacity development in their respective regions and, as importantly, improve the visibility of developing country concerns in the international climate change policy discourse (Runci 2007: 225).

The importance of having traditional knowledge valued and heard both from within Pacific communities and in the international community is strongly advocated by some writers. As Papua New Guinea’s Minister for Community Development, Dame Carol Kidu states, “There is a need for empirical, traditional knowledge and scientific knowledge to connect with each other and to be discussed at the same forums" (Kidu 2011: 1). Huffer and Qalo support this,

Using concepts understood by people at all levels of society will help make leaders more accountable. While terms such as good governance, the rule of law, democracy, human rights, development, and so on are largely seen as impositions from outside and are seldom understood, long standing local concepts embody ideals of social justice, welfare for all, services for the people, and other values in ways that make sense to and empower local people (Huffer \& Qalo 2004: 87).

Valuing traditional knowledge allows for those who have been portrayed as most vulnerable to have a platform from which they are heard. As Ronald Jumeau, Seychelles Ambassador to the United Nations, frames it,

The fight against climate change is a fight based on our undeniable human right to exist, and not just as nation states, but as peoples and communities. The people of the Arctic, for example, and other indigenous peoples are also suffering climate change first hand. Their voices, just like those of the SIDS [Small Island Developing States] and other particularly vulnerable states, need to be heard by the world’s leaders (Jumeau 2009: 5).

These arguments support the critiques of objectivity and emphasise the importance of cultural understanding, which impacts upon the creation of knowledge. It would appear that the lack of representation of Pacific people within the IPCC will have a direct impact on how climate change research is understood, valued and incorporated into policies both by Pacific people and by the international community. 
Thus far I have discussed the literature examining who is producing the knowledge within the IPCC. It is also salient to examine the work from the Pacific. Barnett and Campbell highlight several coordinated Pacific research programmes, which have produced research on climate change from as early as the late 1980s, noting,

Researchers from the Pacific Islands were relatively quick to address issues of climate change and their implications for the region. A preliminary report was prepared by members of the Association of South Pacific Environmental Institutions (ASPEI), which first reported to SPREP [Secretariat of the Pacific Regional Environment Programme] in mid 1988 (Barnett \& Campbell 2010: 70).

As Finucane points out, "Pacific islanders have responded successfully to climatic variability for many centuries, in part because of their ability to understand, record, and forecast conditions” (Finucane 2009: 3-4). Representing the Pacific Delegation at the Asia Pacific Congress of Women in Politics (CAPWIP) 2011, Hona Holan-Hako of the Women's Collective (Bougainville Women for Peace and Freedom), states,

Despite the vulnerability of Pacific island countries to the impacts of climate change and disasters, research shows that women and men of Pacific island communities have successfully utilized specialised knowledge of their environments to mitigate disasters through the management of their natural resource bases for generations. (HolanHako 2011: 1).

Holan-Hako points out that this has been done through passing down of knowledge of traditional and cultural practices through generations.

It is critical, however, not to romanticise images of the Pacific. While, as has been shown, there is a wealth of knowledge within the Pacific that at present is not being utilised, there is also evidence that cultural and traditional practices to mitigate disasters are not enough in the face of catastrophic events such as the Samoan tsunami. The Ambo Declaration, a resolution expressing grave concern, was developed at Kiribati's Tarawa Climate Conference in 2010. Pacific countries challenged the lack of climate change funding for adaptation and mitigation strategies. The Declaration was signed by 18 leaders from both within the Pacific and countries like China. This is another example of the Pacific taking the lead on climate change responses. (Climate Change in Kiribati 2010: 1) 
Developing countries' capacity to continue to partake in climate science is strained. Runci notes the limitations that developing countries have in relation to funding for such research projects.

Perhaps the most obvious factor that influences developing countries’ participation in the international community is a chronic constraint to funding. Because developing countries are, by definition, less wealthy than industrialised countries, they have fewer resources available both for the performance of research and for scientists' participation in international scientific expert meetings, conferences, and professional societies (Runci 2007: 225).

Despite countries such as Kiribati having limited ability to fund climate research, there has been a considerable amount of research that has been done about and for Pacific nations. Critics of this research have again commented that the one-sided nature of who is directing the research creates a subjective form of knowledge. Researchers such as Kandlikar and Sagar note that the majority of funding for such research is being provided by the north. They claim that "This biases the structure, participation and direction of international research efforts, and reinforces the divide between the capabilities of the South and the North” (Kandlikar \& Sagar 1999: 131).

Kandlikar and Sagar are not alone in this analysis of potential biases in western funded research. Haas questions the objective nature of this type of scientific research which he argues should be interpreted using broader cultural discourses. Haas observes,

In part scientific findings may reflect the bias of sponsors, but more deeply they may reflect the broader culture of society from which they emerge and about which they may not be fully conscious (Haas 2004: 572).

Authors such as Kandlikar, Sagar and Haas are not only critiquing the nature of objective scientific knowledge, they are also highlighting the possible impact that this bias may have on both the research and the possible solutions that are sought in relation to the research.

In Barnett and Campbell's assessment of Pacific-centred research, they assert that this is precisely what has occurred. Financial constraints have led to a majority of research being undertaken by outsider researcher bodies. This is perceived by Barnett and Campbell as creating a research scenario dictated by supply rather than demand 
and which also devalues the knowledge that Pacific Islanders have in relation to climate knowledge (Barnett \& Campbell 2010: 82). Barnett and Campbell argue, "More problematically, rarely does this research adequately value the knowledge that people living in the region have about climate, the effect of climate variability and change on their lives and livelihoods, and ways to adapt” (Barnett \& Campbell 2010: 82).

Despite all this, Pacific countries have continued to be vocal advocates for themselves and their region and have brought the climate change issue, and the effects that the Pacific will face, to the world stage. As Weart argues, "Among the governments, the most eloquent and passionate in arguing for strong statements were representatives of small island nations, who had learned that rising sea levels could erase their territories from the map” (Weart 2003: 161). Countries within the Pacific have used both their own knowledge and the knowledge that has been generated from the North to actively push for international action before the homes of Pacific Islanders are irredeemably impacted by climate change. One of the ways that Pacific people have done this is by appealing to the international community for action based upon the perceived image of the Pacific's relative smallness in relation to the rest of the world.

\section{Vulnerability Discourses}

Identification of particular places and people as disproportionately vulnerable, based on both the predicted impacts of climate change and the ability to respond to these projected impacts, has proliferated in climate change literature. Analysis of vulnerability as a descriptive term within climate change research has garnered its own subsection of research that examines the context in which vulnerability discourse is being invoked. A 1981 article by Peter Timmerman is an early example of this work. Timmerman argues that vulnerability discourse is at the centre of climate change research. He references the World Meteorological Organization Climate Program to illustrate this argument, discussing their strategy of "determining the characteristics of human societies at different levels of development which make them either specifically vulnerable or specially resilient to climatic variability and change” (Timmerman 1981: 3). Timmerman goes on to analyse the ways in which vulnerability discourse is being used and argues that, due to its broad use, it has become an unhelpful term except to provide a broad framework for identifying those 
areas most at risk (Timmerman 1981). The broad meanings applied to the term vulnerability and its persistent use within climate research has led many others to also analyse the contexts in which vulnerability discourse is being used and the question of who is using it.

Scholars such as geographer Olivia Warrick have highlighted that the presence of so many disciplinary backgrounds and competing schools of thought contributing to the climate change debate, including scientists, social scientists, economists, and political scientists, has contributed to competing conceptualisations of vulnerability (Warrick 2011). Warrick's argument that these different backgrounds all bring differing contexts of vulnerability into the climate change debate is important, as each school will also bring a different view of the best policy solutions for dealing with climate change and assisting those who have been identified as most vulnerable. Geographer Susan Cutter underscores this point in her article "Vulnerability to Environmental Hazards," where she argues, "Many of the discrepancies in the meaning of vulnerability arise from different epistemological orientations (political ecology, human ecology, physical science, spatial analysis) and subsequent methodological practices” (Cutter 1996: 530).

Despite the apparent divergence of different disciplinary and epistemological orientations for assessing vulnerability, Cutter argues that interpretations of vulnerability can usefully be grouped into several general frameworks. Cutter categorises these as vulnerability based on "risk/hazard exposure; vulnerability as social response; and vulnerability of place” (Cutter 1996: 530). Cutter further characterises each of these three frameworks of vulnerability by elaborating their differing starting position for assessment of vulnerability. In Cutter's analysis, the risk/hazard understanding of vulnerability assessment is based on biophysical hazards and the impact that these hazards may have on a particular place. Social response frameworks diverge from the above understanding of vulnerability in the sense that they assess vulnerability by examining coping responses, including societal resilience to hazards. In this context, vulnerability is contextualised within a social construction framework that takes into account historical, cultural and economic factors that impinge on a state's ability to respond. Lastly, Cutter argues that vulnerability of place frameworks are a mixing of the above two categories but with a more geographically focused assessment of vulnerability (Cutter 1996: 532-533). 
Each of Cutter's three frameworks can be seen to offer distinctive differences in understanding and assessing vulnerability. All three have found their way into the climate change debate and all have relevance to policy solutions. However, current articulations of vulnerability appear to be drawing particularly from what Cutter describes as the vulnerability of place framework. This is evidenced by the IPCC's definition of vulnerability in which they state, "Vulnerability to climate change refers to the propensity of human and ecological systems to suffer harm and their ability to respond to stresses imposed as a result of climate change effects. The vulnerability of a society is influenced by its development path, physical exposures, the distribution of resources, prior stresses and social and government institutions” (IPCC 2007: 17.1). This definition of vulnerability can be seen to be drawing on both biophysical and social construction frameworks of vulnerability.

Understanding the differing frameworks of vulnerability in relation to climate change is imperative as each understanding leads to different policy solutions. The Governance and Social Development Resource Centre, in a bid to make explicit the importance of clarification of vulnerability discourse in the climate change context, notes the policy implications of the differing understandings on vulnerability.

Thus in the scientific framing, the focus tends to be on developing technical responses at the sectoral level based on probable scenarios, whereas the social approach focuses on building the capacity of social groups and institutions to cope with a range of potential stresses and changes, including, but not limited to, climatic changes (Governance and Social Development Resource Centre 2011: 1).

This statement highlights the importance of understanding the slipperiness of the term vulnerability and its use within the climate change debate. This literature review argues that the impact of vulnerability discourse is not just semantic, but also affects policy outcomes dependent on the definitions that are being followed. What becomes clearer in this literature is that biophysical understandings of vulnerability have tended to lead to policies of mitigation, that is the reduction of greenhouse gas emissions, whereas social concepts of vulnerability have leaned more towards adaptation, which focuses instead on how best to adapt to the current and expected changes that climate change is predicted to have, such as by building sea walls, for example. 
Policy responses to climate change are not based on scientific fact alone, and the political mood of the international community also appears to play a pivotal part in creating policies for dealing with a changing climate. Lead researcher for the Climate Change Adaptation Research Group, James Ford, argues that, particularly during the 1990s, academic and political focus was on mitigation with relevant policies put forward that were focused on the reduction of greenhouse gas emissions, which it was argued was the cause of climate change. This can be seen in such international agreements such as the Kyoto Protocol, which is largely focused on mitigation. Ford asserts that this predominance of mitigation strategies is based on two views of climate change policy responses: the first that adaptation policies do not address the underlying cause and that by focusing exclusively on adaptation the underlying cause will not be addressed. Ford highlights former US Vice President Al Gore's statement in 1992 "[Adaptation is] a kind of laziness, an arrogant faith in our ability to react in time to save our skins” (Ford 2008: 7). While Gore's statement indicates a level of dismissal of adaptation as a plausible policy solution, it is apparent that adaptation responses were still being considered. The second view Ford outlines as an explanation for mitigation strategies being most prevalent is based on the political environment of the 1990s in which climate negotiations took place and argues that adaptation was seen by many developed countries as acknowledging human responsibility, which led to fears of liability and compensation being brought into discussions (Ford 2008: 7). It is interesting to note that arguments over responsibility and climate finance/compensation are currently at the centre of discussions for developing countries that are facing the impacts of climate change. The framing of responsibility is considered in more depth in subsequent chapters.

While Ford argues that during the 1990s mitigation was the main focus of climate change policies, he also highlights the growing awareness by both scientists and government bodies that adaptation is a response that needs to run concurrently with mitigation. This has been born out of the awareness that, even with mitigation policies in effect, impacts of climate change are still going to be felt which require policy responses. Development Studies scientist Lisa Schipper claims that "Adaptation to climate change is considered especially relevant for developing countries, where societies are already struggling to meet the challenges posed by existing climate variability” (Schipper 2007: 3). Schipper also introduces a second argument with regard to the increased attention that adaptation policies have received. 
Schipper notes this shift towards adaptation was created by a growing, "frustration over the lack of progress and effectiveness of policy to reduce greenhouse gas emissions...” (Schipper 2007: 3). While mitigation remains a policy response at the international level as both scientific and political knowledge has developed, there has been a clear shift in which adaptation is now seen as a necessary response alongside, rather than in opposition to, mitigation policies. Geographer Diane Liverman claims that "recent discussions of global environmental change have suggested that concern about social causes and consequences of environmental degradation beyond one's own country or locality is in the national and individual self interest" (Liverman 1990: 28). Liverman argues that this is based more on the imperative of political stability rather than ethical responsibility. Liverman asserts, "Reinforcing the ability of other countries to cope with environmental problems is seen not only as a way to develop strategic allies, but also as a means to insure the stability of import export trade” (Liverman1990: 28). Liverman, Schipper, and Ford's arguments highlight the impact that the political environment has on both the climate change debate and policy initiatives.

As has been demonstrated in the preceding section, literature examining discourses of vulnerability has focused on the definitions of vulnerability that are being used to assess the impacts of climate change on people and to respond to climate change through policy initiatives. What emerges in this literature is a consistent reminder that climate change debate is also largely influenced by the politics of the time, which has a direct influence on how vulnerability discourses are being brought into the debate. However, as will be shown in the following section of this literature review, conflict over vulnerability discourse extends beyond the competing definitions that are being used to assess vulnerability. For some, vulnerability itself is a contested term.

\section{Small Island States}

Images of the Pacific Islands' relative smallness have proliferated through much of the literature on climate change. As anthropologist Wolfgang Kempf, writing with regard to discourses about climate change in the Pacific, highlights, “...nowhere have small islands, small land masses, small island states and micro states been talked of with such frequency, or with such a degree of self-evidence, than in this part of the world” (Kempf 2008: 195). These images of smallness have been linked 
simultaneously with images of vulnerability, particularly when talking about the impacts that the Pacific will face and also the Pacific's ability to respond to climate change. This constant reference to smallness has led to an uncontested picture of the Pacific as being small and powerless within the global climate change debate. This is evident in numerous reports published by the IPCC. For example, in the fourth assessment report, "Impacts, Adaptation and Vulnerability,” the IPCC states, Many small islands are highly vulnerable to the impacts of climate change and sea-level rises. They comprise small landmasses surrounded by ocean...in tropical areas they host relatively large populations for the area that they occupy, with high growth rates and densities. Many small islands have poorly developed infrastructure and limited natural, human and economic resources...Most of their economies are reliant on a limited resources base and are subject to external forces, such as changing terms of trade, economic liberalisation, and migration flows. Adaptive capacity to climate change is generally low, though traditionally there has been some resilience in the face of environmental change (IPCC 2007: 16.2.1).

This statement from the IPCC illustrates how geographical smallness is being used to identify the perceived vulnerability of places such as Pacific Islands. It is also clear in these evocations of 'smallness' that it is not just their landmass that makes these islands small but also their geographical and economic position in relation to the rest of the world. These factors have all been used to reiterate the vulnerability of the Pacific Islands.

When looking at the literature on Pacific Islands, Kiribati has been identified as one such island nation that is at particular risk due to the ongoing effects of a changing climate. This vulnerability to climate change is often articulated through images of the nation's smallness, both in terms of its geographical makeup and also its economic ability to respond to climate change. The IPCC is not alone in this articulation of Kiribati's vulnerability to climate change based on its geographical and economic smallness. Maryann Loughry and Jane McAdam, writing in a chapter looking specifically at Kiribati in a report by the Refugee Studies Centre, point to a range of factors contributing to Kiribati's vulnerability, including its 
Low-lying land mass with the population having no recourse to higher lands, the nation's limited sources of income, and the concentration of the majority of the population on one dominant atoll. These factors combined with increasing changes in climate, pose a threat to Kiribati's food and water security, health and infrastructure, as well as the ability of the Kiribati government to cope with increasing climate-related disasters (Loughry \& McAdam 2008: 51).

The relationship between Kiribati's geographical dimensions and the risks that it faces due to climate change, such as continued sea level rise, has largely been uncontested by either international bodies or research commissioned by Pacific institutions. The Climate Institute expresses it thus

Kiribati is extremely vulnerable to climate change, climate variability and sea level rise. The 33 atolls of Kiribati are less than 500-1,000 m (average $450 \mathrm{~m}$ ) in width and rarely surpass 3 meters above sea level, and Tarawi [Tarawa], which contains half the national population, lies less than 3 meters above sea level (Climate Institute. 2011: 1).

Research conducted by the Tyndall Centre for Climate Change similarly argues, Atolls have common environmental problems that render them particularly vulnerable to climate change... Overall their small size, isolation, physical infrastructure and low levels of income make atoll countries apparently vulnerable to economic forces as well as to climatic changes (Barnett \& Adger 2011: 3).

The Government of Kiribati has also outlined the country's extreme vulnerability to rising sea levels due to climatic change that Kiribati faces, claiming, "We are among the most vulnerable of the vulnerable. Even a marginal increase in sea levels will be disastrous for our country's future” (Kiribati Government 2011: 1). A report produced by the Japan Ministry of the Environment and the South Pacific Regional Environment asserts,

Pacific Island Countries may well be among the first to suffer the adverse impacts of climate change, and the first to be forced to adapt. Most countries are already experiencing disruptive changes consistent with many of the anticipated consequences of global 
climate change, including extensive coastal erosion, droughts, coral bleaching, more widespread and frequent occurrence of mosquito-borne diseases, and higher sea levels making some soils too saline for cultivation of traditional crops (Hay et.al. 2011: 12).

Such comments highlight the impact that climate change is already having on islands such as Kiribati due to their geographic makeup and the urgency for island nations such as Kiribati to adapt to these changes.

However, the discourse of vulnerability that has become synonymous with the Pacific, and Kiribati as an island group within the Pacific, has been contested. Scholars have noted the impact that such discourses of vulnerability have had on the image of the Pacific. Barnett and Campbell, writing in regard to vulnerability discourse, describe it this way,

...vulnerable entities are defined in terms of their opposites in the binary: things that are vulnerable are not powerful, large, robust and knowing, but are weak, powerless, and fragile and naïve...These characteristics imply then that the large and powerful can and should act to help the helpless from their predicament since vulnerable cannot by definition act to help themselves. Thus vulnerability discourses are a form of knowledge/power: they represent the world in ways that serve the interests of power (Barnett \& Campbell 2010: 163).

This analysis of vulnerability discourse emphasises the significance of the choice of description, and its consequences.

Barnett and Campbell are not alone in this analysis of vulnerability. Pacific-based theorists have countered the notion that smallness implicitly implies vulnerability, and that the Pacific is both small and therefore vulnerable. Greg Fry, in his analysis of how the Pacific is portrayed within the Australian context, asserts that historic and current depictions of the region have a direct impact not only on the way that the Pacific is understood by Australia, but also matters to those Pacific peoples who are being spoken about. Fry argues,

It has mattered for Pacific Islanders when, at various times over the past two hundred years, influential Australians have viewed them collectively as savages, children, or full human beings, and whether 
the region was depicted as a defence shield, a frontier, vulnerable, empty, or unstable (Fry 2000: 26).

Fry goes on to state that these images have affected the ways that the Pacific has been treated, arguing,

Each of these lenses allowed or encouraged different Australian behaviour toward Pacific Islanders: from colonial control and exploitation, to protection, development, and the encouragement of self-determination. (Fry 1997: 307).

Fry's point is an important one: the particular lenses that are used when talking about the Pacific impact on the way that Pacific Islands and Islanders are then treated. Focusing exclusively on Pacific Islands' vulnerability, based on their financial position and geographical dimensions, allows little room for acknowledgment that the very reason that islands such as Kiribati are facing such dramatic outcomes as a result of climate change has very little to do with them. Kiribati, for example, produces a comparatively very small amount of carbon emissions compared to western nations. Despite the mitigation strategies that it has implemented and can implement, Kiribati remains at risk due to western nations' continued actions (and inaction).

Epeli Hau'ofa, an influential figure in Pacific Studies literature, has been very critical of the persistence of the international community's approach and view of the Pacific. He states that the Pacific has been framed as consisting of small, dependent, fragmented islands that are reliant on international aid and development for survival. Hau'ofa asserts, "It is a belittling view that has been propagated unwittingly-mostly by social scientists who have sincere concern for the welfare of Pacific peoples” (Hau'ofa 2008: 29). Hau'ofa goes on to argue,

According to this view, the small island states and territories of the Pacific, that is, all of Polynesia and Micronesia, are too small, too poorly endowed with resources, and too isolated from the centres of economic growth for their inhabitants ever to be able to rise above their present condition of dependence on the largesse of wealth nations (Hau'ofa 2008: 29).

Hau'ofa's first critical presentation of this framing of the Pacific was published in 1993, and it has remained potent and oft-cited since that time. As Kempf notes, Hau'ofa's essay has remained a foundational piece of Pacific critique that is consistently drawn upon to challenge belittling views of the region. Drawing on 
Hau'ofa's framing of the Pacific for this research is appropriate because, despite the fact that when it was written, climate change was not the public concern it is today, the very language that Hau'ofa warns against is once again appearing with frequency. It is almost impossible to find reference to the Pacific and climate change without finding reference to Pacific Islands' smallness, isolation, dependency and vulnerability. It is for this reason that Hau'ofa's work remains a pivotal text for examining the portrayal of the Pacific today. Kempf demonstrates that Hau'ofa's model, despite having been critiqued by commentators (Waddell Naidu \& Hau'ofa 1993), continues to engage and have relevance to this day (Kempf 2008).

Echoing the influential work of Edward Said, Australia-based Pacific Studies scholar Katerina Martina Teaiwa points out that the image of the Pacific as small, isolated and therefore vulnerable and powerless is an image that is produced as a counterpoint to the 'west's' view of itself. Teaiwa claims,

There is still, however, much in the rhetoric of globalization and development discourse that asserts the continuing smallness, peripherality, instability and helplessness of small island states and groups of mainly indigenous peoples (Teaiwa 2005: 173).

Teaiwa goes on to say, “The language of development 'experts', aid agencies and good governance projects coming out of countries like Australia in particular often historically conflates powerlessness with peripherality” (Teaiwa 2005: 173). These critiques of how the Pacific is viewed by the international community imply that using discourses of vulnerability will negatively affect the way that Pacific issues are dealt with.

However, discussions of vulnerability within the climate change debate offer an interesting paradox when we consider the use of these images by representatives of islands within the Pacific. Imagining the Pacific as a set of small vulnerable isolated islands has been extremely productive for government officials, activists and NGOs trying to draw attention to islands such as Kiribati; such discourses have been actively mobilised to propel these islands into the international community's sights. Wolfgang Kempf makes a case for the rhetorical power of "smallness" in debates about climate change.

[Smallness] need not always carry negative implications. Precisely in view of the fact that many islands are at acute risk from climate change and rising sea levels, smallness should be seen, I think, as 
constituting one of the most important argumentative resources in the political discourse of those contemporary Pacific societies that find themselves facing the prospect of forced migration and resettlement (Kempf 2008: 196).

Kempf highlights how representatives of Pacific Island states have wielded a discursive recourse to "smallness" in their active petitioning of "Leading industrial countries, as the primary agents of the global green house effect, to step up to the plate and shoulder their moral responsibility." Kempf concludes that, "In this environment of economic, social and political inequality, smallness for these disadvantaged island societies is a useful argumentative resource in the fight to secure great equity of burden sharing” (Kempf 2008: 197).

The productive use of discourses of vulnerability is evidenced, for example, in the work of Maria Tiimon, the Kiribati-born, Australia-based Pacific outreach project officer for the Pacific Calling Partnership, who is responsible for raising awareness of climate change issues for both the Pacific communities living in Australia and the wider Australian public. In a speech delivered to a recent Berlin conference on climate change and the Pacific, Tiimon repeatedly invoked the image of Kiribati as a small island state, beseeching the audience,

I am begging industrialised countries to have mercy on us and help us. Please give us your hand and let [us] do this together. Once again, please we need your help, we are vulnerable, so please do not ignore climate change. We are small islands. If no one is listening to us, there will be devastation: our land and our beautiful culture will be under the water (Tiimon 2011: 4).

Statements such as these highlight the way in which the imagery of the Pacific as a region of small dependent states has also been adopted by those who represent islands in Governmental and non-governmental sectors in order to try and galvanise the international community into action. As Barnett and Campbell put it, "representations of the islands as being vulnerable to climate change have been helpful in leveraging international support: the construction of the small islands as Davids fighting against the industrial and newly developing Goliaths has considerable popular appeal in the developed world” (Barnett \& Campbell 2010: 155). However, while acknowledging the salience of such depictions, Barnett and Campbell ultimately question the degree to which popular appeal has been translated into action: "However, this support has 
resulted in little in the way of material action, either in the form of a reduction in greenhouse gas emissions or the implementation of programmes that would help the Pacific island governments and communities build effective adaptation strategies” (Barnett \& Campbell 2012: 155).

Barnett and Campbell's conclusion highlights the tensions that arise in the continued use of vulnerability as a descriptive term applied to Pacific Island states. As has been shown, the term has attracted considerable criticism but it has also been adopted by Pacific Islands spokespeople as a way of gaining public, political and media attention in the climate change debate. Discourses of vulnerability appear, paradoxically, to both legitimate and delegitimate the subject being described. Is this a productive way of categorising island states such as Kiribati, and what will the ongoing consequences be from the persistent invocation of their 'vulnerability'?

\section{Forced Migration as a Consequence of Climate Change}

As a result of climate change, islands such as Kiribati have been identified as extremely vulnerable to rising sea levels, causing coastal erosion and various other consequences. These consequences, if severe enough, would make Kiribati unliveable and forced migration is therefore a very real threat. Images of 'sinking islands' and islands such as Kiribati being swallowed by the sea have proliferated in climate change literature and have captured the imagination of those reporting on the looming disasters caused by climate change. Such images have highlighted that forced migration is a pressing issue for both the Pacific Islands at risk and for the international community who are engaged with responding to these issues. International organisations have produced a plethora of literature about the eventuality of forced migration as a result of climate change (IPCC 1990; 2007; Asian Development Bank 2011; Refugee Studies Centre 2008). In the first report published by the IPCC in 1990, they highlight the risk that islands such as Kiribati face due to climate change, stating,

Many small island countries would lose a significant part of their land area with a sea level rise of $1 \mathrm{~m}$. Coral atoll nations, such as Kiribati, Marshall Islands, Tokelau and Tuvalu, Cocos and Keeling islands, are particularly vulnerable to inundation and erosion because they are generally below $3 \mathrm{~m}$ elevation and narrow, implying few possibilities for retreat (IPCC 1990: 63). 
The risk that small islands face led the IPCC to warn that "Sea level rise impacts on low lying Pacific island atoll states of Kiribati, Tuvalu, Tokelau and the Marshall Islands may, at some threshold, pose risks to their sovereignty or existence” (IPCC 2007: 87).

Since that time, numerous international bodies have repeatedly referenced the threat of forced migration due to climate change. Achim Steiner from the United Nations Environmental Programme states, "Human migration, forced or otherwise, will undoubtedly be one of the most significant consequences of environmental degradation and climate change in decades to come” (Steiner 2008: 1). Oli Brown from the United Nations Development Programme supports this, summing,

Put simply, climate change will cause population movement by making certain parts of the world much less viable places to live; by causing food and water supplies to become more unreliable and increasing the frequency and severity of floods and storms (Brown 2007: 1).

This view that Pacific Island states such as Kiribati are extremely vulnerable to climate change, possibly resulting in forced migration, is not restricted to international organisations. Many statements from Pacific governmental representatives and organisations reiterate this position. President Tong of Kiribati asserts, "The science is clear, climate change threatens the long-term survival of Kiribati. As such the Kiribati Government acknowledges that relocation of our people may be inevitable” (Office of the President of Kiribati 2011: 1). Peter Emberson, speaking on behalf of the Pacific Conference of Churches, argues, "Climate change will result in the loss of life, land, and liberty. It is our prophetic responsibility to comprehensively address issues confronting the realities of Resettlement as a consequence of climate change" (Emberson 2010: 24).

However, tensions emerge in the literature in relation to the focus that is being placed on forced migration as the final response to climate change. Some have argued that the attention being paid to forced migration diverts attention from other mitigation and adaption strategies that could be employed earlier. The Asian Development Bank puts forward the argument that,

Too often it is assumed that all environmental impacts will result in displacement migration. For example, in the atoll Pacific countries, the discussion on potential sea-level rise has focused 
exclusively on resettlement migration as a response, such that other forms of mitigation and adaptation have been neglected (Asian Development Bank 2011: 8).

Such criticism of identifying forced migration as an inevitable scenario, and the consequential lack of other forms of strategic planning, is reiterated by John Campbell, who comments,

The most popular image of climate change effects in Pacific island countries is that of small islands, particularly atolls, being inundated ('drowned'). Indeed, inundation poses significant threats to coastal and atoll communities. However, for many such communities, other effects may also force communities to take adaptive action and indeed may occur sooner than the effects of sea-level rise (Campbell 2010: 31).

This tension has led some to question the imagery of vulnerability in relation to the Pacific and forced migration. Barnett and Campbell, when questioning whether the use of vulnerability discourses in relation to the Pacific has been useful or not, suggest,

At least with respect to adaptation responses, representation of the Pacific islands as extremely vulnerable may have created the illusion that adaptation is pointless, and have denied the resilience, agency, capacity and potential that the Pacific island communities have and which could be useful elements of an adaptation response (Barnett \& Campbell 2010: 155).

Human geographer Carol Farbotko, writing about Tuvalu, analyses the way that Pacific Islands are portrayed in climate change debate. In particular, Farbotko critiques the sedimentation of discourse about Tuvalu's imminent "sinking”, arguing compellingly that,

In the cosmopolitan gaze, there is a strong, albeit paradoxical connection in the politics of the global climate change crisis between 'saving the climate refugee' and 'watching the islands drown'. Island people, long marginalised, are denied their own agency in the climate change crisis. They are fictionalised into victim populations fleeing inundation, desperate for dry land, even drowned (Farbotko 2010: 58). 
Farbotko in this passage vividly identifies the infantilising images that appear frequently in the discourse.

As has been shown in the above review of the literature, there is a clear tension around drawing on images of vulnerability and the effect that this may have on the international community's responses to the climate change issues facing island nations such as Kiribati. Further differences emerge in forced migration literature about the specific factors that will force people to move from their homes. The United Nations High Commissioner for Refugees, Susin Park, reinforces the likelihood of forced migration. She argues that climate change can sometimes be the final straw for already vulnerable societies, and that consequences of climate change — such as rising sea levels spoiling arable land and extreme weather eventswhile not causing the sinking of islands, would make them unliveable. Park argues,

In most cases, such a risk is likely to arise due to a confluence of economic, social, geological and environmental factors, where climate change may constitute the tipping point. Such factors would be likely to cause extensive displacement regardless (Park 2009: 1-2).

Statements such as these highlight the perceived twofold threat to islands such as Kiribati, one based on the impacts of climate change, the second based on the economic situation of Kiribati for which people may choose to migrate irrespective of climate change. These arguments are synthesised by Oli Brown who states, "Migration, even forced migration, is not usually just a product of an environmental push from a climate process like sea level rise. Except in cases of climate events, where people flee for their lives, it does require some kind of pull: be it environmental, social or economic” (Brown 2008: 19). Migrants may be pushed and/or pulled, but what of their characterisation in the debate?

Tensions emerge between the foundational writing of Epeli Hau'ofa and the current framing of discussion about forced migration due to climate change when the act of migration, itself, is considered. In his oft-cited critical essays, Hau'ofa characterises the people of the Pacific as natural voyagers, with centuries-old migration routes: "Theirs was a large world in which peoples and cultures moved and mingled, unhindered by boundaries of the kind erected much later by imperial powers” (Hau'ofa 2008: 33). This image of Pacific Island people as voyagers is echoed in Katerina Martina Teaiwa's work, where she emphasises, "Movement 
within the Pacific along specific itineraries characterises much of the social, political and economic life of islanders in both the past and the present” (Teaiwa 2005: 173). This vision of free and unfettered movement around the region stands in sharp contrast to tragic images of "climate refugees." Other scholars researching the Pacific have also suggested that migration — even when compelled by circumstances beyond the control of migrants-may present opportunities as well as challenges (Campbell 2010).

While migration due to climate change may not necessarily need to be viewed as negatively as it is often framed, an important distinction is required between voluntary migration, which implies an element of choice, and forced migration, which implies no choice. This thesis intends to bring important and necessary feminist and Pacific studies critical theory to bear on discourse about forced migration due to climate change, in hopes of productively negotiating out of an impasse between absolute narratives of victimhood and agency. It is precisely because the stakes of these issues are so high that careful interventions in the language we use to understand them are necessary.

\section{Women and Vulnerability}

Women's visibility in climate change debates has become a pivotal concern for those looking at women's particular vulnerabilities to climate change. Literature highlighting the extreme vulnerability of women in the context of climate change is plentiful, and concludes that the likely outcomes for women are different from those for men. These arguments have in turn led to demands for gender sensitive policies to address women's needs within the climate change debates.

Research has identified that the economic situation of a population has a direct correlation to how vulnerable they are to the effects of climate change. As globally women are disproportionately represented amongst those living under the poverty line, it follows that they will be among the most vulnerable of the vulnerable. (Haigh \& Vallely 2010). Jyoti Parikh, Director of Integrated Research and Action for Development, points to Gro Harlem Brundtland's observation about those most vulnerable to climate changes. Brundtland states,

Poor people are more vulnerable to climate change due to their limited adaptive capacities to a changing environment. Among them, the rural poor, and rural women and girls are the ones most 
immediately affected. Climate change impacts are not gender neutral (Brundtland quoted in Parikh 2009: 2).

These arguments regarding women's vulnerability based on their economic situation are further developed in literature with a specific focus on women which additionally explores several other factors that contribute to women's vulnerability. In a statement issued by the Center for Asia Pacific Women in Politics it is stated,

Climate change is not a neutral process; first of all, women are, in general, more vulnerable to the effects of climate change, not just because they represent the majority of the world's poor but also because they are more than proportionally dependent on natural resources that are threatened... (Center for Asia Pacific Women in Politics 2008: 2).

Parikh further highlights women's disproportionate vulnerability to climate change arguing,

Women are affected differently, and often more severely than men, by climate change and natural disasters such as floods, droughts, cyclones and storms due to gender-based discrimination against women, unequal power relations between women and men. Including in access to assets and resources, as well as intrahousehold inequalities and related vulnerabilities, capabilities and opportunities for adjustment (Parikh 2009: 2).

Literature supporting arguments such as Parikh's is plentiful, with women's inequality in decision-making processes consistently highlighted as an additional explanation for women's extreme vulnerability. Again, the Center for Asia Pacific Women in Politics states, "An overall assessment of climate change debate to date shows women are patently absent in the decision-making process. (Center for Asia Pacific Women in Politics 2008: 2). They illustrate this argument by noting that policy formulation in regard to such matters as conservation and environmental management are the preserve of men. Rose Mensah-Kutin, Regional Programme Manager for ABANTU for Development, acknowledges the exclusion of women's voices from decision-making processes, but rather than focusing on how this increases women's vulnerability, she introduces the argument that women have vital knowledge that needs to be included. According to Mensah-Kutin, 
The need for a gender responsive approach to climate change policymaking is imperative. This is because excluding the voices of women from climate change discussions means excluding the voices of half the world's populations, denying them rights and ability to contribute vital knowledge (Mensah-Kutin 2010: 27).

This statement, while noting the exclusion of women's voices, presents the argument for inclusion of women's voices from a different angle. It focuses on the abilities that women can bring to the table as opposed to merely highlighting how exclusion exacerbates vulnerability. Philippe Boncour, from the International Organisation for Migration, and human rights lawyer Bruce Burson build on this argument and also introduce the impact that gendered roles play in increasing women's vulnerability. When migration is considered, Bouncour and Burson claim,

In general women are expected to be particularly vulnerable to the impacts of climate change as a result of existing gender inequalities that limit their access to information and decision making power. This increased vulnerability is also a function of women's frequently insecure property rights and access to resources, as well as of their reduced mobility in situations of environmental stress, because of their childcare and eldercare responsibilities (Boncour \& Burson 2010: 14-15).

Statements such as these illustrate both women's lack of involvement in decisionmaking processes and their gendered roles within society, such as child and elder carers, as factors that increase their vulnerability.

Commentators on Kiribati have identified forced migration as an issue. It is important therefore to examine the literature about the gendered effects that may result. Commentators looking at migration due to climate change have noted that the ability of people to migrate is largely skill dependent. Literature examining the gendered impacts of forced migration has therefore focused on the different roles that men and women play within a society and the impact this then has on women's particular vulnerability. Looking at forced migration, specifically, Boncour and Burson further emphasise female vulnerability, concluding,

It is clear, however, that not everyone can use migration as an adaptation strategy: it depends on resources, information, and other social and personal factors. Often, it is precisely the most 
vulnerable and most severely affected who are not in a position to migrate. More specifically, differentiated gender impacts must be expressly factored into the policy-making process (Boncour \& Burson 2010: 14-15).

In a report published by the Women's Environment Network, Christine Haigh and Bernadette Vallely highlight how women's roles within society make them most vulnerable when talking about forced migration. Haigh and Vallely state,

Migration may be of whole families or communities, but in many cases a single individual from a family, usually (although not always) male, will migrate in order to find alternative livelihood. In such cases, women who are left behind tend to have increased care and domestic responsibilities, which may compromise their ability to do income-generating work (Haigh \& Vallely 2010: 14).

Literature such as this highlights the research that is being conducted on the impacts that women will face due to climate change, with women's unique vulnerabilities constantly being highlighted to demand policy action that factors in women.

The vulnerability of women emphasised in both the general climate change literature and specific forced migration literature has been heavily relied upon as a way of promoting women's inclusion within climate change debates and the development of adaptation strategies and policy solutions. In much the same way as was highlighted earlier in relation to the literature on the vulnerability of small island states, the term 'vulnerable' has increasingly become a tool used to garner attention for women within the climate change debates. As Ulrike Rohr, co-founder of the NGO Gender CC Women for Climate Justice indicates,

When many development organisations entered the climate change arena, they emphasised the issue of adaptation and were (of course) referring to the vulnerability of women. The result: seeing women as victims or most vulnerable in society provided a foot in the door in the climate change discussions (Rohr 2011: 1).

However when examining arguments about why it is vital for women to be included within climate debates, it becomes clear that there are contrasting views of how women are being portrayed. A dichotomy between characterisations of vulnerability versus characterisation as change agents appears to be prevalent amongst this literature. 
Critical thinking about the oft-invoked term 'vulnerability', in relation to women within the climate change debates, has led some researchers to critique discourses of vulnerability as a primary justification for women's inclusion within climate change discussions, debates and policies. Anita Nayar, writing for Development Alternatives with Women for a New Era (DAWN), specifically challenges rationales that argue for including women in policy only because of their vulnerability. Nayar is critical of language that depicts women as victims of climate change, arguing instead that women need to be included in policies based on the vital role that women play within a society and as individuals with strengths and abilities to offer solutions" (Nayar 2011: 1). Nayar argues,

The framing of women as a 'vulnerable' group is widespread in the discourse. We need to challenge this and reclaim women's pivotal role in the productive economy. We need [to] recognise women as change agents engaged in the struggles over fossil fuel exploitation, pollution and environmental health...” (Nayar 2011: 1).

Nayar is adamant that any policy developed needs to “address women's and men's different relationships to ecological systems as producer, workers, consumers, conservers, not merely as subjects 'vulnerable' to 'disasters”' (Nayar 2011: 1). Nayar's critique of vulnerability as a reason for including women in climate change policy highlights this conflicting tension between conceptualising women as 'change agents' versus ‘victims'.

Nayar is not alone in providing critical reflection on this tension. Sherilyn MacGregory, a political scientist, highlights this continued use of vulnerability as a descriptive term for women within the climate change debate. MacGregory articulates the conflicts that arise when continuing to paint women as solely vulnerable within this arena,

The most frequent co-location of words in the gender and climate change research is undoubtedly 'women' and 'vulnerable'... These images and portraits are problematic, I would argue, because the result is that rural women in the south are constructed as onedimensional objects: they rarely enter the discussion unless as climate victims. By focusing on vulnerability, there is a danger of affirming the negative stereotype (perpetuated by the media in the 
north) of the southern women as helpless, voiceless and largely unable to cope without the help of UN development agencies funded and staffed by the north (MacGregor. 2010: 289).

MacGregor's comments bring to light the problems that may face women if they continue to be solely identified as a vulnerable group. In addition, viewing a group of people through a particular lens impacts upon the choice of policies constructed to meet that group's needs. These arguments are echoed by Ranjani K. Murthy. Writing on the topic of disaster legislation, Murthy critiques that “...[women] are often seen as solely 'vulnerable' groups, with their participation in shaping disaster policies or monitoring their implementation from a gender or [Sexual and Reproductive Health and Rights] SRHR lens not being envisaged” (Murthy 2000: 12). Blomstrom, Cunningham, Johnson and Owren similarly state,

Women are sometimes seen only as victims of climate change and natural disasters, when in fact they are well positioned to be agents of change through mitigation, management and adaptive activities in their households, workplaces, communities and countries. Women can be effective leaders within their communities when it comes to addressing the harmful effects of climate change... Their efforts must be incorporated into climate change policies and promoted through capacity building (Blomstrom, Cunningham, Johnson \& Owren 2009: 4).

Statements such as these bring into question the way in which women are being depicted throughout climate change literature. Just as has been highlighted in previous sections of this literature review, there is a clear tension emerging around the prevalent use of vulnerability as a descriptive term for both the Pacific Islands, generally, and Pacific women, specifically. Irene Dankelman, environmental activist, confronts this tension within academic writing and points out, "For me there is no contradiction (contrast) between being 'vulnerable' and being an agent of change: even the most vulnerable women and children can be very inspirational agents of change...” (Dankelman 2011: 1). Dankelman goes on to restate her position on the women that are so often being drawn upon to colour researchers' arguments, claiming, "They are not vulnerable - but they are made vulnerable by different external drivers.” (Dankelman 2011: 1). Ulrike Rohr, co-founder of Gender CC Women for Climate Justice, takes this further and argues, 
It is not desirable to shift from one extreme: vulnerability of women is the main gender issue, to another: women are agents of change. This leads to a romanticisation of women, based on women as individuals, rather than questioning their societal roles and responsibilities...The idea of the 'Agents of Change' is a myth. It shifts societal responsibilities, e.g. for transitioning to a low carbon society, onto individuals to those having the least impact on political decisions (Rohr 2011: 1).

In much the same way as island states are cast as particularly vulnerable, the term vulnerable has increasingly become a tool that is being used to garner attention for women within the climate change debates. If the term vulnerability is an adequate characterisation of Pacific Islanders and women within the climate change debate, then, by extension, that would indicate that Pacific Island women are the most vulnerable of the vulnerable-first as Pacific Island peoples and second as women.

These contrasting opinions of how women are being and should be framed also highlight the conflicts that arise when looking at the question of on what basis women should be included within climate change debates. To address the needs of women it would appear that it is vital for women to be included within climate change debate; how to include women to provide the best outcomes is what is in contention. Acknowledging women's position within society and their vulnerability due to this position is important; however this cannot be at the expense of inclusion of women in climate change debate and policies as equal members of society.

\section{Conclusion}

What has become clear throughout this literature review is that the term 'vulnerable' is heavily drawn upon in climate change literature to describe the position of both the Pacific Islands and women. Applying both Pacific studies and feminist theories to analyse the term vulnerability within the climate change debate, it is apparent that there are tensions relating to the use of the term for both Pacific Islands and women. As has been shown, who is producing knowledge about climate change, and the lack of Pacific Island knowledge and voices included in reports from international bodies such as the IPCC, have impacts upon the way that the Pacific is viewed. The scientific evidence that shows that Pacific Island states such as Kiribati are at extreme risk to climate change is not disputed. However what has been highlighted is that 
terms such as small, which are being used to indicate vulnerability, are disputed by some. This critical analysis of vulnerability and Pacific Islands, I would argue, highlights differences that are produced depending on which worldview is being drawn upon. As demonstrated in the literature review, the Pacific Islands are often cast, from an international standpoint, as small isolated islands vulnerable to climate change. However Pacific theorists produce a radically different image of the Pacific Islands. This can also be seen in the literature on forced migration as a consequence of climate change. Although forced migration is a possible outcome that Pacific Island nations such as Kiribati may face due to climate change, the heavy reliance on discourses of vulnerability has been argued to create a sense of inevitability of 'sinking islands'. Throughout this literature review, vulnerability discourses are ever present when examining climate change and the impacts for the Pacific Islands and for women. In relation to women within the climate change debate, commentators advocate that there needs to be more inclusion of women, yet on what basis is still being contested. In conclusion this literature review has highlighted the heavy use of vulnerability as a descriptive word for both women and islands, and raised questions regarding the work that vulnerability discourses do. Subsequent chapters further analyse how this term impacts on climate change debates. 


\section{Chapter Three: Outline of Methodological Approach}

The intention of this thesis is to examine discourses of vulnerability and explore the possible impact that such discourses have on I-Kiribati women, using forced migration due to climate change as a case study to ground this research. To accomplish this, this thesis examines bodies of scientific and social scientific research about climate change and forced migration, as well as public debates, and activist discourse. In particular, I analyse how discourses of vulnerability are invoked in these literatures, how discourses of vulnerability frame and shape discussions and debates, and the tensions produced between discourses of vulnerability and agency. I employ a cultural studies approach that incorporates a strategy of discourse analysis guided by both Pacific studies and feminist theoretical trajectories.

A key component of undertaking research in Pacific studies is valuing the knowledge that is generated by Pacific Island peoples. As scholar Houston Wood argues, "the emerging cultural studies for Oceania rejects claims that western science and disciplines possess epistemologies superior to local epistemologies found throughout Oceania...the emerging cultural studies for Oceania demand multiple epistemologies” (Wood 2003: 354). In order to apply this to my own research, I will be using Pacific studies, indigenous feminist and other feminist frameworks to critique the current debates and literature that are being produced about climate change, Kiribati and I-Kiribati women. Feminist theory also situates the researcher within the research and such reflexivity will also be a key feature of my research methodology.

\section{Why Qualitative Research?}

The aim of this research project is to understand the ways in which discourses of vulnerability are being utilised in relation to Kiribati and I-Kiribati women within the climate change debate, and the possible implications such discourses have on policy, debate and action. This research, then, demands that the term 'vulnerability' be explored within the political context in which it is being used; it seeks to uncover meanings for the individuals that are being termed in this way and the meanings that those who are drawing on such language are ascribing to it. Such a focus makes qualitative research methodologies appropriate. As sociologist Jennifer Brayton explains, "Qualitative research commonly refers to the collection and the analysis of 
material that seeks to uncover meaning and understanding of experience...Qualitative is thought to value subjective, personal meaning and definition, commonalities and giving voices to the oppressed” (Brayton 1997: 1). John Creswell, a professor of educational psychology who specialises in research methods, explains further, "Qualitative research is exploratory...this type of approach may be needed because the topic is new, the topic has never been addressed with a certain sample or group of people...” (Creswell 2009: 18).

Existing research has thus far examined the likely outcomes of climate change on places such as Kiribati, the differential impacts that these outcomes may have on women, and the use and definitions of vulnerability discourse being brought to bear in the climate change debate. My research differs from the above research in that its focus is to examine how vulnerability discourses are being invoked within the climate change discourse with specific attention being paid to Kiribati and I-Kiribati women. Chapter four begins by exploring the terms that are being used by the international community to talk about Kiribati and women. It is within this chapter that issues of power, history and politics are brought to bear through the analysis of language and questions are explored such as how this language effects the construction of forced migration as an eventual policy option? Chapters five and six examine articulations of women and Kiribati from the perspective of two I-Kiribati women who are active in climate change discussions and action. The purpose is to examine if and how these women articulate the vulnerability of Kiribati and I-Kiribati women in differing ways, and what this means for the way forced migration is viewed and expressed. As all three of these chapters are intending to examine the meanings that are being attached to the discourse of vulnerability, the application of Brayton's analysis of qualitative research appears most applicable. The intention of this research is to both critique the use of this language and explore the power dynamics that arise in the use of this language within the climate change debate. This research will then outline how language that is being drawn upon by both the international community and Alofa and Tiimon is similar or different and in what ways this influences the framing of Kiribati, I-Kiribati women and forced migration. This has not been done previously, which makes my research exploratory.

A qualitative research approach also allows me to adhere to aspects of feminist research methodologies in order to produce a piece of feminist research. Feminist researchers have typically tended towards qualitative approaches as these 
methodologies allow for women's experiences and voices to come through the research. However, applying qualitative research methods to research does not guarantee the result to be feminist research. Marjorie Devault, a sociologist specialising in gender studies, points out, "qualitative methods practiced in non feminist ways can easily reproduce the mainstream failure to notice women and their concerns” (DeVault 1996: 33). In order to make sure that this research is feminist in nature, as opposed to women-centred, I have chosen to apply several key strategies, which will be examined in this chapter.

\section{A Case Study Approach}

The design of this research project is based on what Norman Denzin and Yvonna Lincoln identify in their widely-cited work on qualitative research methodologies as a case study strategy (Denzin \& Lincoln 2005). Rather than being a methodology, the case study is an approach that guides the researcher in the selection of what to study and the tools to use to enable in-depth research on a particular subject. In Hagan's analysis of the case study approach, he stipulates that the case study method implies “In-depth, qualitative studies of one or a few illustrative cases” (Hagan 2006: 240). As is outlined below, the case study approach has shaped the nature of this research based on two of Denzin and Lincoln's defining features of the case study.

The first rationale for applying the case study approach to this research is that a case study strategy allows the focus of this research to be situated based on a geographic location. The second rationale is that the case study approach allows for multiple sources of material to be used. Considering the first rationale of situating this research within a geographic boundary I point to Robert Stake, writing about the choice made by researchers to use a case study approach, who states, "We will all have a research question, a puzzlement, a need for general understanding, and feel that we may get insight into the question by studying a particular case” (Stake 1995: 3). Prior research clearly indicates that climate induced migration is a possible outcome for several locations across the globe and within the Pacific (IPCC 2007; Louhry \& McAdam 2008; Barnett \& Adger 2011). Prior research also highlights that women are indicated as being particularly vulnerable to climate induced migration across the globe (Boncour \& Burson 2010; Haigh \& Vallely 2010). However, in order to adequately interrogate discourses of vulnerability, it appeared a necessary choice to 
narrow the research scope geographically in order to be able to explore in the necessary depth.

It is also imperative, however, to note criticisms of case studies as a methodological approach to research. Terence Wesley-Smith, in his seminal work on the different research approaches that have been employed when researching the Pacific, has identified what he describes as the "laboratory rationale.” In his analysis of the laboratory rationale, he argues that researchers have used the Pacific as a laboratory to test hypotheses (Wesley-Smith 1995). Wesley-Smith notes criticisms of this approach in that it leads Pacific Island peoples to be viewed as objects of interest and does little to incorporate Pacific ways of knowing into the field of research (Wesley-Smith 1995). These criticisms outlined by Wesley-Smith highlight the dilemmas in applying a case study approach. In order to avoid these shortcomings in the application of a case study approach in this research, it is imperative to underscore the awareness of this researcher that the Pacific is not a homogenous set of islands in which research produced can be presented as representing the whole Pacific region and therefore this research is not intended to be universalised.

The criticism presented by Wesley-Smith in the above section holds similarities with feminist critiques of research being presented as universally applicable. Feminists, and particularly non-white and/or non-western feminists, have often critiqued the presumptions made around essentialist notions of sisterhood. Rather than presuming that women face universal forms of inequality, they argue instead that there are vast differences based on geography, class and race that all impact on how inequality is perceived and felt. Feminists such as Patricia Hill Collins present the argument thus, "We conceptualise difference as a range of interlocking inequalities, where individuals experience categories or positionality differently depending upon their social locations within the social structures of their given society" (Collins in Nagy Hesse-Bieber \& Leckenby 2004: 214). This statement reiterates the shortfalls of a research focus that uses a specific geographical space or group of people to make broad, universalising assessments. It is clear that difference needs to be factored into research and specific findings are not assumed to be universal.

Taking these critiques into consideration for my own research, I contend that the use of a case study still has relevance. Robert Stake (1994) categorises case study research into three distinct research types: intrinsic, instrumental and collective. Stake outlines the fundamentals of each approach, defining intrinsic case study as that in 
which a particular case is given or chosen and the focus of the research is case specific. Stake defines the instrumental case study as when we seek to answer a general question and feel by choosing a particular case it will enable insight into answering the broader research question. And finally, the collective case study is defined as where several cases are used to gather data and then correlated to answer a particular question (Stake 1995). I contend that this research is based on the intrinsic case study model. Berg elaborates Stake’s rationale for researchers undertaking intrinsic case studies: "Intrinsic case studies are undertaken when a researcher wants to better understand a particular case. It is not undertaken primarily because it represents other cases or because it illustrates some particular trait, characteristic, or problem” (Berg 2009: 325). This research is not intended to test the reality of broader theories of vulnerability discourse and its effects, using Kiribati as a geographical space to do this. Rather this research is specifically focused on investigating how vulnerability discourses are entering the debate about forced migration due to climate change in the Kiribati context, and what limitations and opportunities are presented by the way current discourse is framed.

As this is such a substantial piece of work to undertake, the choice to narrow this down and examine these discourses with particular reference to Kiribati and I-Kiribati women allows me to examine the issues in more depth whilst grounding my findings within a particular case study. As Bruce Berg points out, "By concentrating on a single phenomenon, individual, community, or institution, the researcher aims to uncover the manifest interaction of significant factors characteristic of this phenomenon, individual, community, or institution. But, in addition, the researcher is able to capture various nuances, patterns, and more latent elements that other researcher approaches might overlook" (Berg 2009: 318). Following this characterisation, then, the case study approach will allow this research to examine the complications and problems of vulnerability discourse in a more analytical and indepth manner by narrowing the focus to Kiribati and I-Kiribati women.

The second rationale of the case study approach is that case study research argues for the use of multiple sources for gathering data. Robert K. Yin, a pivotal figure in writing about case study methodological approaches, defines the case study research method as "an empirical inquiry that investigates a contemporary phenomenon within its real life context, when the boundaries between phenomenon and context are not clearly evident, and in which multiple sources of evidence are used” (Yin 1984: 23). 
As Tellis notes, "Case studies are multi-perspectival analyses. This means that the researcher considers not just the voice and perspective of the actors, but also of the relevant groups of actors and the interaction between them” (Tellis 1997: 1). By augmenting the literature that is produced about Kiribati and I-Kiribati women's vulnerability with key voices from within Kiribati, I will be able to compare and contrast the use and meanings that are being applied in discussions of vulnerability in these differing settings.

In order to bring in the voices of key players from within Kiribati, I will be interrogating published documents, speeches, media publications and information from websites, which will be used to provide a counterpoint for what is being written about Kiribati and I-Kiribati at the international level. Both Pelenise Alofa, National Coordinator for Climate Action Network (CAN), and Maria Tiimon, are vocal advocates for bringing to light the issues that Kiribati faces in relation to climate change. As they are both such prolific producers of written and oral advocacy, a specific focus on their work allows me to analyse their contributions to discourses of vulnerability by looking at published material as opposed to interviewing them.

\section{Critical Discourse Analysis}

This research is focused on analysing vulnerability discourse and its application to Kiribati and I-Kiribati women, both by those outside of Kiribati and by two I-Kiribati women. Questions are asked about the meanings attached to the term vulnerability, what impact these have on those who are being spoken about in this way, and, in a related vein, how forced migration is viewed and articulated. This research seeks to uncover the ways in which vulnerability discourses are being utilised within the climate change debate, whether these usages have an impact on how women are being included within current debates and policies, and the ways that activist discourses may be shaped by, and shape, scientific and popular discourses and policy.

Such close attention to the discursive construction of knowledge about Pacific places and peoples has been particularly salient for research within the Pacific studies paradigm. Scholars situated in the region, such as Epeli Hau'ofa and Greg Fry, have critiqued the use of language in academic and political discussion about the Pacific, and have commented upon the impact that language has. Hau'ofa, an influential figure in Pacific Studies literature, has argued that language that emphasises the idea of Pacific nations as small and with few economic resources reinforces the idea of 
dependency, which is disempowering for Pacific nations and does little to acknowledge or value the resources that the Pacific has. Hau'ofa has been very critical of the persistence of the international community's approach and view of the Pacific. He argues that the Pacific has been framed as small, dependent, fragmented islands that are reliant on international aid and development for survival. Hau'ofa argues, "It is a belittling view that has been propagated unwittingly—mostly by social scientists who have sincere concern for the welfare of Pacific peoples" (Hau 'ofa 2008: 29).

Greg Fry contends that depictions of the Pacific by non-Pacific researchers, policymakers, and media have influenced the treatment of islands and islanders by the international community (Fry 2000). Following the work of Edward Said, Fry contends that examining such depictions not only provides insight into how the Pacific is portrayed, but also how the 'framers' see themselves. Writing specifically about the Australian context, Fry argues that the frames through which the Pacific is imagined contain and maintain inherent power relationships and have influenced the ways in which the Pacific has been treated. Fry elaborates, "Each of these lenses allowed or encouraged different Australian behaviour towards Pacific islanders: from colonial control and exploitation, to protection, development, and the encouragement of self-determination” (Fry 2000: 2). Critiques such as these encourage and guide this thesis as it seeks to situate the impacts of vulnerability discourses within geographical and temporal contexts.

Discourse analysis is a field where close attention is paid to how language is used. Within the broad field of discourse analysis there are a range of approaches that can be followed; for the purpose of this research it was felt that critical discourse analysis was the most salient form of analysis to apply. As Teun Van Dijk, a scholar in the field of critical discourse analysis, explains, "Critical discourses analysis (CDA) is a type of discourse analytical research that primarily studies the way social power abuse, dominance, and inequality are enacted, reproduced and resisted by text and talk in the social political context" (Van Dijk 1998: 1). The application of critical discourse analysis for this project allows discourses of vulnerability to be examined within the political context in which they are being used. Norman Fairclough and Ruth Wodak underscore the fundamental importance of discourse for shaping how the world is understood and acted upon. 
...discourse is socially constitutive, as well as socially conditioned - it constitutes situations, objects of knowledge, and the social identities of and relationships between people and groups of people. It is constitutive both in the sense that it helps to sustain and reproduce the social status quo, and in the sense that it contributes to transforming it. Since discourse is so socially consequential, it gives rise to important issues of power. Discursive practices may have major ideological effects - that is, they can help produce and reproduce unequal power relations between (for instances) social classes, women and men, and ethnic/cultural majorities and minorities through the ways in which they represent things and position people (Fairclough \& Wodak 1997: 357-358).

Using this elaboration of discourse then allows for the exploration of not only where discourses of vulnerability are being used, but also examination of who is drawing on this term, how, for what reason, and to what extent terms such as vulnerability and vulnerable have effects for the people that they are being applied to.

Chapter four uses this model of exploration to examine the ways that Kiribati and I-Kiribati women are being spoken about from outside of the region. The language that is being used is analysed from a standpoint where the political and historical depictions of both islands and women can be explored and challenged. It is the intention of Chapter four to illustrate the ways that vulnerability discourse is being used to convey messages of dependency based on an innate quality of both Kiribati and I-Kiribati women. Questions are examined such as: how does this language impact on the people being spoken about in this way?; what are the underlying meanings and understandings that are being brought into the climate change debate?; and, how does this shape the way that forced migration is thought about, talked about and presented within the climate change debate? Chapters five and six then examine the way that both Alofa and Tiimon articulate images of Kiribati and I-Kiribati women. The focus of these chapters is to examine the way that Alofa and Tiimon bring both Kiribati and I-Kiribati women into the climate change debate examining the similarities or differences in the language they use and exploring whether this has an impact on the way that Kiribati, I-Kiribati women and forced migration are considered. 
Undertaking this type of critical discourse analyses requires that the researcher draw attention to, explore, and analyse issues of power that may normally be veiled in the use of vulnerability discourses. In "Critiquing the critical: A reflection on critical discourse analysis,” Donald Matheson warns the reader that critical discourse analysis can be criticised for reproducing the very problems it seeks to address in the analysis of power and meaning, in the sense that the researcher's analysis is always informed by the researcher's own understandings and positionality. Matheson cautions, "Critical discourse analysis, because it is explicitly bringing external perspectives to the language events it is studying, faces particularly sharply the accusation that it is misreading or over-reading its object of study in terms of the analyst's own politics” (Matheson 2008: 84). Taking this criticism on board, it is particularly relevant to make explicit the epistemological background of the researcher; this can be achieved through the incorporation of reflexivity in this research.

\section{Reflexivity}

The importance of reflexivity when conducting critical discourse analysis has been made explicit in Matheson's argument above. This critique of critical discourse analysis raises important questions regarding the notion of objectivity. The key premise of objectivity is that it is value free; according to this supposition, the need for the researcher to locate oneself within the research and the research process is unwarranted, as researchers can arrive at the absolute truth without being influenced by the researcher's personal position. Feminists, however, strongly object to the notion of objectivity. As Nicole Westmarland sums, "Feminists have broadly rejected the idea of methods premised on the idea of 'objectivity' being used to measure social knowledge” (Westmarland cited in Maynard 1994: 16). This position then argues for the acknowledgement of subjectivity; that is, to acknowledge the subject. Feminists such as Mary Maynard argue "the researcher is also a subject in her research and that her personal history is part of the process through which understanding and conclusions are reached” (Maynard 1994: 16). This argument that the researcher impacts on the research from the very beginning has seen feminist researchers strive for the acknowledgement of the subjective influence in the research process. As Ramazanoglu argues, "It is more logical to accept our subjectivity, our emotions and our other socially grounded positions than to assume some of us can rise above them (cited in Westmarland 2001: 1). By acknowledging that I, as a researcher, will be 
bringing my own position to the research, I allow for critics, readers and other academics to situate the research that is gathered through the research process. As this research draws heavily on both Pacific and feminist theory to inform my analysis of vulnerability discourse within the climate change debate, it is imperative to acknowledge the way that this has informed both my interpretation of the discourse and the shaping of my arguments. As a researcher I am very much aware that with the application of different theoretical understandings, the research could take a different form and reach different conclusions.

Attention to reflexivity is also a key element shared by both feminist and indigenous feminist research methodologies. This is an important element of how I structure, analyse and present my research. In order to make explicit the power relations that are inherent in any research project, feminists have argued that reflexivity is a pivotal tool. It allows power relations to be explored and made explicit, and identifies how this may have impacted on the research. For feminist researchers such as Caroline Ramazanoglu, reflexivity is a way of accounting for the power relations within the research process. (Ramazanoglu \& Holland 2002). As has already been addressed, the application of critical discourse analysis presents issues of interpretation. By making explicit the position that the researcher holds, it not only allows for transparency within the research process, it also allows for the positionality of the researcher to be acknowledged and critiqued. Mauthner and Doucet explain the purpose of reflexivity as, "[acknowledging] the nature of the research relationship, and the extent to which similarities or differences between researcher and researched in characteristics such as gender, race, class, age, sexuality, or able bodiedness influence this relationship” (Mauthner \& Doucet 1998: 121). This process allows the researcher to be aware of differing situational inequalities that may impact on the research process and outcome of the research. This is particularly important in the analysis of both Alofa's and Tiimon's articulations of I-Kiribati women's vulnerability. As a woman from New Zealand, my knowledge of how race and gender issues are thought about and then articulated is restricted by my own experience. By applying the method of reflexivity it has allowed me to consider how my own positionality impacts on the analysis of this research, and to bring in both indigenous and indigenous feminist critiques to examine the way that Alofa and Tiimon articulate or overlook I-Kiribati women's position with climate change. 
In the same vein, the examination of power balances/imbalances requires the researcher to examine the impact of insider/outsider research. Maykut and Morehouse describe the insider/outsider dimension of research as one that needs to be considered within a fluid notion of what constitutes as insider/outsider. They argue, "The qualitative research perspective is perhaps a paradoxical one: it is to be acutely tunedin to the experiences and meaning systems of others... and at the same time to be aware of how one's own biases and preconceptions may be influencing what one is trying to understand (Maykut \& Morehouse 1994: 123). This analysis of the insider/outsider impact on research has particular relevance for this research and researcher. As the researcher it can be argued that in the examination of how women are being spoken about, my identity as a woman positions me as an insider. This identity as a woman and as a feminist has influenced the importance that I have placed on how women are being spoken about within the climate change debate. This standpoint has led me to argue for the importance of having women included within any and all climate change discussions and policies. However, as a pākeha woman I have limited insight into how race and nationality intersect with gender and what influence this has on how gender is thought about from the position of I-Kiribati women. I have therefore drawn heavily from indigenous feminist analysis of race and gender to inform my conclusions about the way Alofa and Tiimon incorporate women into their discussions.

This is similarly important for indigenous methodological approaches, which argue for reflexivity to be used when unpacking the insider/outsider dimension when undertaking research. (Tuhiwai-Smith 1999). The acknowledgement of the insider/outsider dimensions of research allows the researcher to further locate oneself within the research process and examine issues of power that arise throughout any research process. As the researcher I have the power to shape this research, choose what to include and not include, what analytical tools will be used in the analysis of the research and the ultimate presentation of this research. McEwan, in talking about one of the key premises of postcolonial feminism, identifies the power that researchers have in speaking for and about women in the south (McEwan 2001). Considering this critique of western feminist presumption to speak for and about women, I have attempted to bring in the voices of both Alofa and Tiimon. Feminists have considered ways to offset the power imbalances that arise during the research process. Brayton, for example, suggests, "The unequal power relationship between 
the researcher and the subject is restructured to validate the perspective of the participant” (Brayton 1997: 5). By bringing Alofa and Tiimon's voices into this research I have attempted to allow them to speak for themselves. I have done this through the use of the speeches and presentations that are within the public domain. This choice was a conscious decision based on an awareness of the frustration that IKiribati have felt about the substantial number of researchers flying into Kiribati undertaking research and then flying out again. Due to time constraints, it was felt that in order to undertake research that was in line with indigenous methodological values, conducting interviews would not be the best way to include Alofa and Tiimon's voices as I would not have the time needed to undertake them appropriately. I am aware that speeches given on behalf of or to particular organizations, in particular fora, may be constrained or shaped by certain necessities of language, tone, or argumentation (see Riles 2001). I have tried to offset this by gathering a range of Alofa and Tiimon's speeches at different venues and to different audiences. While there may be some small shift in what they are saying, by and large their discourse remains consistent despite the venue, audience or media outlet.

When considering the power balances within the research process it is also imperative to consider the ability to empower through research. Empowerment is a key premise of both feminist and indigenous methodological approaches. Just as feminist research methods demand that research start from a premise of women being at the centre of research with a particular focus on research that empowers women, indigenous methodologies also start from a premise that indigenous people are at the centre of research (Tuhiwai Smith 1999). Research that is centred on indigenous people, it is argued, will enable any research that is undertaken to be based on empowerment. As Jelena Porsanger notes in her article about indigenous methodological approaches to research, "Indigenous methodologies require scholars to think critically about their research processes and outcomes, bearing in mind that indigenous peoples' interests, experiences and knowledge must be at the centre of research methodologies...” (Porsanger 2004: 109). This is one of the key concerns in the application of indigenous methodologies, as it is argued this is a way of countering historical research practices which have seen indigenous people's inclusion in the research process being limited to subjects, as opposed to indigenous peoples having ownership over any research process. 
This requirement to, as a researcher, take responsibility for ensuring that indigenous peoples are at the centre of research requires the researcher to apply a level of reflexivity over their research process. Linda Tuhiwai Smith presents what she believes are fundamental questions that need to be asked of both the research and the researcher, including but not limited to, "Whose research is this?... Whose interests does it serve? Who will benefit from it?...” (Tuhiwai Smith 1999:10). As this research is a discourse analysis using publicly available speeches, testimonies and statements made by both Pelenise Alofa and Maria Tiimon, the question of who this work belongs to takes on a more abstract meaning. It is my hope that this work can be seen as belonging to both the women that I have drawn from and also to a wider audience who can critically engage with my analysis. By applying indigenous and other feminist analysis to the language that I am examining, and conducting my research with an awareness of how colonisation and issues of power and inequality are present within this language, I hope that my work brings attention to and understanding of these issues. 


\section{Chapter Four: Articulations of Islands, Women and Forced Migration from Outside the Pacific}

The representation of certain places and people as particularly vulnerable to climate change is ubiquitous within climate change literature and discussions. As was previously noted in Chapter two, vulnerability as a descriptive term has undergone significant analysis and critique, with arguments attempting to clarify what is meant when vulnerability is attached to particular places and people. These discussions have brought several competing and intersecting notions of vulnerability from a range of disciplines to the fore of climate change debate. The heavy reliance on vulnerability categorisation, and the importance of clarifying what is meant by vulnerability, can be seen in the IPCC's later reports where explanations of vulnerability are provided to make explicit how vulnerability is being assessed and what is meant by vulnerability.

When we look at portrayals of the Pacific within the climate change debate, it is clear that vulnerability discourses are drawn upon to express the consequences of climate change and as a way of urging the international community to act. The categorisation of women as particularly vulnerable has been a way of not only urging the inclusion of women in policy and discussions, but also as a way to highlight broader gender inequalities within society. Although, as shown in Chapter two, these views of women as vulnerable are contested and debated, nevertheless there remains a heavy reliance on vulnerability discourse as a way of categorising the impacts of climate change on women.

This reliance on vulnerability discourses suggests that, as a descriptive term and a categorising term, vulnerable is a useful and productive way of labelling places and people. While Chapter two has highlighted that, in regard to women, the term vulnerability is contested within some-particularly feminist-discussion, when the conversation turns to climate change, people inevitably fall back on 'vulnerable' as a descriptor. This recourse to vulnerability as a descriptive term prompts some complex and challenging questions that require further analysis. In examining Kiribati's vulnerability, key questions to examine include: how does framing Kiribati in this way impact on the view of Kiribati's future in the long term? What are the inherent power balances/unbalances that are reinforced when drawing on this type of 
language? Further, does this language categorise the position of Kiribati within climate change debates in a particular kind of way, and if so, how?

Using Kiribati as a case study to ground an examination of the consequences of drawing on vulnerability discourses, this chapter will seek to answer these questions. In order to do this I will examine the co-location of terms that are being used to give shape to vulnerability discourses and how these terms provide a fuller image of Kiribati, and other Pacific Islands, as innately vulnerable. Next I will look at the inclusion of women in vulnerability discourse about forced migration. I critically examine the parallels between the way women and Pacific Islands are characterised. Lastly this chapter explores how vulnerability discourses are affecting the way in which forced migration has come to be shown as an inevitable outcome of climate change for Kiribati. Although it is not disputed that Kiribati already faces and will continue to face some serious ramifications from continued climate change, the presumption of the eventual forced migration of the whole of Kiribati has become so interwoven with concepts of vulnerability that questions need to be asked about what impact this may have on shaping the international response to climate change and, in particular, forced migration. This chapter explores some of the ways that this plays out within the international literature and discussions.

\section{Articulations of Islands}

The convergence of so many disciplines in climate change research has introduced multiple understandings of vulnerability. As has already been noted, this mix of backgrounds has led many scholars to argue for clarification of the meanings of vulnerability that are being employed. Scholars such as Nick Brooks have asserted that this can be as easy as providing clarifying terms that make explicit the meaning of vulnerability that is being applied. Brooks argues, "Such clarification can be achieved through practices as simple as the application of an adjective; the confusion arising from different usages of the term 'vulnerability' may be largely overcome by differentiating between 'social vulnerability' and biophysical vulnerability...” (Brooks 2003: 2-3). What becomes clear is that in order to provide these clarifying terms, researchers are drawing on adjectives that create and sustain a particular image of the Pacific as overwhelmingly and intrinsically vulnerable.

As has been shown in the overview of literature on climate change in Chapter two, adjectives such as small, isolated, low-lying, developing and economically dependent 
have all been used in co-location with vulnerability in climate change research and debate. These terms have all been used to emphasise the vulnerability of places such as Kiribati. For example, small, isolated and low-lying are all indicative of biophysical vulnerability as they are referring to the geographic characteristics that make islands such as Kiribati so susceptible to the impacts that a change in climate is predicted to have. References to the economic situation of Kiribati can clearly be linked to social understandings of vulnerability, as they are focused on the ability of people and governments to respond to those biophysical risks.

Although it can be argued that these terms are being used to clarify how vulnerability is being assessed, such categorisation schemes potentially create a false impression that 'biophysical' and 'social' understandings of vulnerability can be discretely and neatly separated. An examination of the literature indicates that terms such as small, isolated and low-lying are referring to more than just the physical nature of particular Pacific Islands. Instead the vulnerability associated with smallness, etc., is largely based upon these Pacific Islands' small economic resources, isolation from international markets and low-lying geographies that impact on their ability to prosper economically. These are seen to create difficulties in managing environmental resources, creating smaller areas of liveable space, leading to problems of overcrowding that deplete resources further. This is evidenced in Pelling and Uitto's analyses of vulnerability in which they present vulnerability diagrammatically, as reproduced here:

Table 1: Intrinsic vulnerability in small island developing states

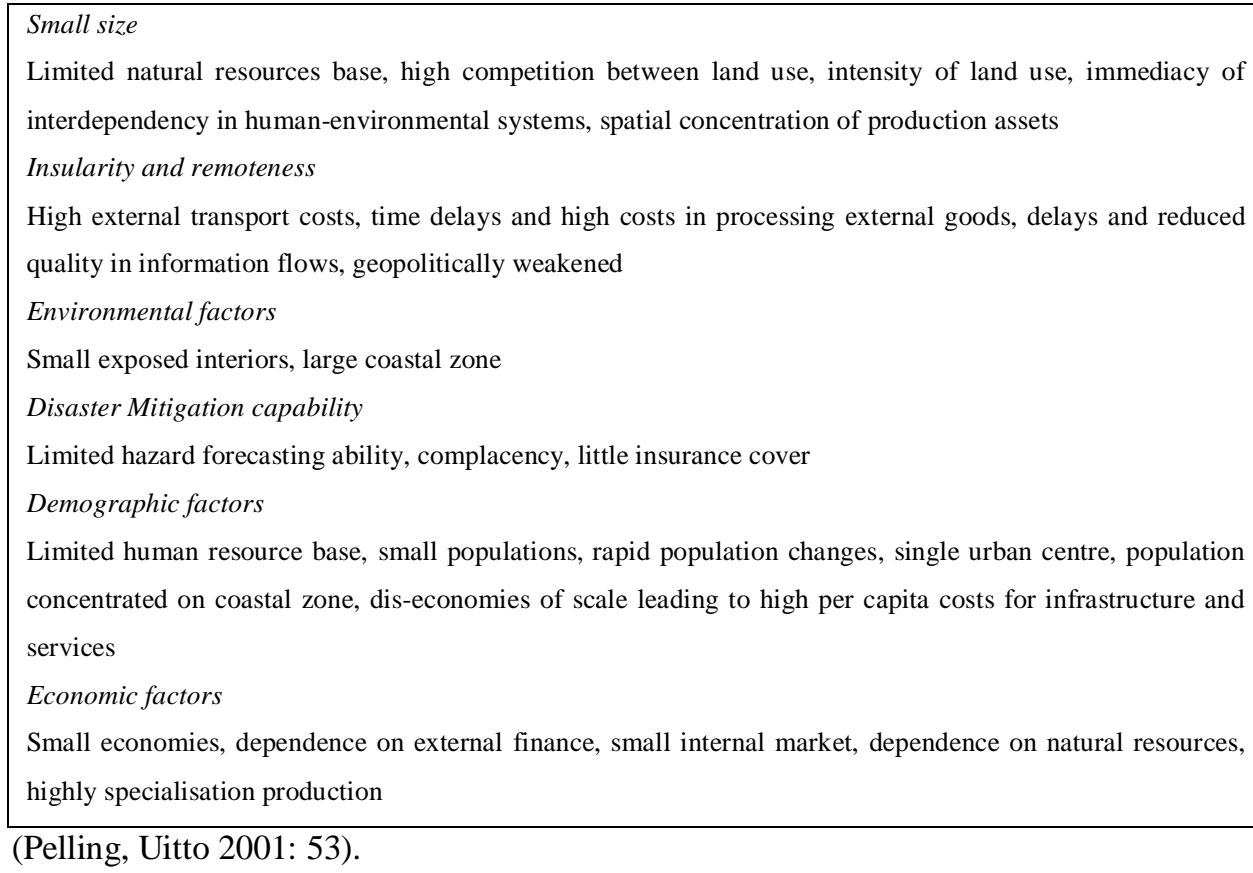


This articulation of vulnerability highlights that adjectives such as small, isolated and low-lying are often referring to Pacific Islands' economic position and isolation from economic markets rather than the geographic makeup of the islands.

This framing of island vulnerability, which draws on clarifying adjectives such as small and isolated, is neither new nor unique to climate change discussions. Lino Briguglio, an economist and author of a seminal article on the inherent economic vulnerability of small island states, provides a framework for assessing vulnerability in which he identifies four key indicators of vulnerability: small size; remoteness and insularity; disaster proneness; and environmental fragility. Each of these factors is heightened by processes of colonisation and globalisation that have positioned Pacific Island states in relation to a global economic market. Briguglio links smallness and isolation to economic vulnerability; he argues that it is through this isolation and lack of a large economic and resources base that islands are particularly vulnerable (Briguglio 1995).

Briguglio and Pelling and Uitto's assessments of vulnerability both underscore the way in which Pacific Island states are being framed as vulnerable within a wider international context. Their economic position is being consistently drawn upon to highlight Small Island States’ particular vulnerability, and by implication, their likely continued dependency as discussed further below. The use of adjectives such as small, etc., attempt to make explicit how vulnerability discourse is being applied, and whether it's biophysical or social vulnerability frameworks that are being drawn upon. However, as has been demonstrated, terms such as small and isolated carry with them understandings and articulations of the Pacific that have little to do with biophysical or social frameworks of vulnerability. Instead they are based on long held views of the Pacific in which the region's vulnerability is expressed as an innate quality determined by the fact that it is composed of island geographies. With the increased attention that climate change has brought to Pacific Islands, these articulations of vulnerability are being reproduced and re-articulated constantly. These descriptions and their regular application to Kiribati reinforce these long-held views of the Pacific's vulnerability that are interconnected with relationships of power and dependency within a global system.

In examining these discourses of vulnerability, it is salient to note that each of these factors is established in relation to an 'other'. Pacific Islands states' smallness and isolation is expressed in relation to an economic epicentre from which they are 
viewed as small and isolated. This articulation of Pacific Islands creates an image of dependency, one in which Pacific Islands are reliant on international markets and western powers in order to be rescued from their vulnerability. Epeli Hau'ofa makes the argument that this view of the Pacific originated with the arrival of western imperialists, with the drawing of imagined borders around the Pacific in order to mark out colonial territories (Hau'ofa 2008). Hau'ofa goes on to reiterate that these markings of smallness and isolation are relative, stating, “...it depends on what is included and excluded in any calculation of size...Their calculation is based entirely on the extent of the land surfaces they see” (Hau'ofa 2008: 31). Hau'ofa is providing a counter argument to smallness from a Pacific perspective, one in which views of Pacific Islands are seen from within and size is based on the surrounding Pacific ocean and the connectedness to other Pacific Islands through trade and kinship networks that long pre-date the era of European, American-and in some cases Asian-imperialism. This argument makes apparent that the imagery of the Pacific as small and isolated, created during the time of colonisation of Pacific territory, has been established using reflective images of the colonisers. The Pacific is small and isolated only in reference to the large, central, and, although not explicitly expressed, powerful colonisers. This holds particular relevance to the climate change literature and the expressions of Pacific vulnerability that literature creates and reproduces.

This articulation of vulnerability can be seen to be drawing on simple dualisms. For example, islands are isolated only in relation to somewhere else, and islands are small as opposed to large. The dualisms that become apparent in the climate change literature are expressed below:

Table 2: Dualisms in climate change literature

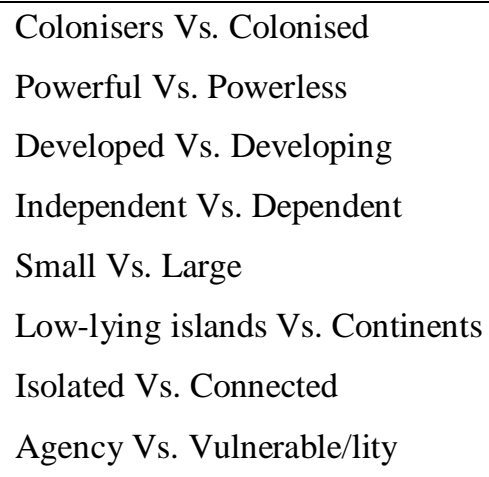

When we compare this with the dualisms that were often brought to bear during colonisation, there are strong similarities between the ways the Pacific was depicted 
during earlier eras of colonisation and now how the Pacific is being depicted through climate change discussions. The climate change debate is drawing on vulnerability discourses that are adhering to this same self-reflective view of the Pacific that enabled the colonisers to act in the ways that they did. Beckett (1987) argues that in order for colonisation to work, colonisers established counterpoints of ideological difference in order to foster views of superiority over those colonised. Differences including race, mentality, moral qualities, cultural advancement or religion were most often drawn upon to highlight the superiority of the colonisers. This depiction of the Pacific in both climate change literature and colonisation literature indicates the way in which framings of the Pacific are mired in issues of power and control (Fry 2000). As Sardar argues, "The real power of the west lies not in its economic muscle and technical might. Rather, it resides in its power to define” (Sardar 1999: 1). When applying this argument to the climate change debate literature, it can be seen that the imagery of the Pacific that was created through colonisation is once again being drawn upon to outline the Pacific's specific vulnerability. Although this may be unintentional, it is in essence binding the fates of Pacific Islands to powerful states that have the power to determine if, and how, to act.

Examining how the vulnerability of Pacific Islands is expressed in relation to climate change requires that power relations be explored. In utilising these adjectives to articulate their vulnerability, there are discursive echoes of previous images of Pacific Islands' vulnerability. This acts to further disempower Pacific Islands by presenting the Pacific as innately vulnerable; and it allows the international community to take little responsibility for both the causes of climate change and the responsibility to act in response to climate change. What this analysis illustrates is that the attribution of adjectives such as small, isolated and developing, although in essence attempting to clarify terms of vulnerability, when examined through the lens of Pacific theories are merely re-articulating and further entrenching long-held views of the Pacific, binding nations like Kiribati into a cycle of power and control.

\section{Articulations of Women}

Pacific Islands' vulnerability has been assessed using biophysical models that draw on categorising terms such as small, isolated and developing. The models employed to define the vulnerability of people are largely based around social vulnerability frameworks that rely on their own categorising terms, such as gender. Adger and 
Kelly's model of social vulnerability allows for assessments of how social institutions interact with biophysical impacts to create vulnerabilities. Neil Adger and Mick Kelly posit that social vulnerability is assessed by, "the capacity to anticipate, cope with, resist, and recover from the impact of a natural hazard” (Adger \& Kelly 2000: 327). Using this model for assessing specific vulnerabilities of people to the impacts of climate change Adger and Kelly argue allows for interrogation to be made of the social structures that limit people's ability to respond to these impacts. Bernadette Resurreccion (2011) points to Piers Blaikie, Terry Cannon, Ian Davis and Ben Wisner's argument that "Vulnerability is a key concept in predicting and understanding the differentiated impacts of various disasters on groups in society, as it takes into account people and the differences among them, affirming that people's circumstances change and can be changed by a disaster” (Blaikie, Cannon, Davis \& Wisner 1994 in Resurreccion 2011: 6). This reliance on social vulnerability frameworks has allowed the impacts of climate change to be understood where different circumstances, economic situations and ability to act are all taken into consideration. Through the application of social vulnerability models, it becomes clear that the impacts of climate change, coupled with social structures, have placed women at the centre of vulnerability assessments.

Critical analyses of gender roles within society have been used to reinforce ideas of women's vulnerability and to argue for gender responsive policies within climate change debates. As has been outlined in Chapter two, women's gendered roles within society have been heavily drawn upon to articulate women's vulnerability, referencing women's roles as mothers and carers, their exclusion from decisionmaking roles and their economic disadvantage (Boncour 2009; Mensah-Kutin 2010; Parikh 2009). For example, Momsen, cited in in Manion's article "Ecofeminism within Gender and Development,” states,

As household managers, women are the first to suffer when access

to sustainable livelihoods are unbalanced. When the water becomes unpotable, the food stores dry up, the trees disappear, the land becomes untenable and the climate changes, women are often the ones who need to walk further and work harder to ensure their families survival (Momsen in Manion 1995: 5).

Momsen's articulation of the gendered impacts that climate change creates is based on the gendered roles that women have within society. Momsen argues that it is these 
roles that place women in a particularly vulnerable position. Such examinations of women's vulnerability that place women's gendered roles at the centre of the assessments litter the literature on climate change and women's vulnerability. This can be seen in articles produced by a plethora of international organisations, such as the United Nations Development Programme’s “Resource Guide on Gender and Climate Change" (2009); the Women’s Environment Network, with their "Gender and the Climate Change Agenda: The Impacts of Climate Change on Women and Public Policy” (2010); and The World Bank’s “Gender and Climate Change: Three Things You Should Know" (2011). All of these publications attempt to make explicit the underlying social structures that create an environment in which women are more susceptible to climate change hazards.

When we examine I-Kiribati women specifically, arguments such as these appear to hold relevance in relation to the positions that they hold within society. A report published by UNESCO demonstrates that I-Kiribati women's involvement in parliament and in Island Councils, which are the national and local government structures, respectively, remains relatively low. The report states, "There has been an increase in the representation of women in parliament; however this remains low with only three female parliamentarians out of 44 in the current parliament. There is limited involvement of women in Island Councils...” (UNESCO 2009: 9). This limited participation by I-Kiribati women in formal decision-making positions suggests that their ability to provide input on climate change response strategies is inhibited. If we follow the arguments that women's participation in decision-making reduces vulnerability, then it can certainly be argued that I-Kiribati women are particularly vulnerable to climate change and its outcomes due to their exclusion from decision-making processes.

However some feminist scholars such as Sandra Grey have raised the question of whether political representation of women truly produces better policy results for women. Questioning whether achieving critical mass in representation will ensure that women's issues are furthered within political arenas, Grey argues, “...Women are by no means a coherent group and every female legislator will have cross-cutting identity characteristics that affects her world views" (Grey 2006: 493). This is a theme echoed by many indigenous feminists who challenge the overarching identity of women as a collective made by western feminists (Trask 1993; Tuhiwai Smith 1999). Heeding these critics raises the question of whether women's vulnerability can be accounted 
for by their lack of representation; simply increasing numbers of women in policy positions may not entail an increase in women's needs being voiced or met. This concept of representation equalling action for women will be explored further in Chapter five when I examine how two I-Kiribati women, Pelenise Alofa and Maria Tiimon, articulate Kiribati’s vulnerability.

As Chapter two demonstrated, women's vulnerability is expressed throughout both climate change literature and vulnerability literature, indicating that women as a distinct group are the most vulnerable of the vulnerable and require particular mention, attention and action in order to protect them (Parikh 2009; Boncour \& Burson 2010). An example of this articulation of women's vulnerability can be seen in this statement made by UN Women Watch.

Women are more vulnerable to the effects of climate change than men-primarily as they constitute the majority of the world's poor...when coupled with unequal access to resources and to decision-making processes, limited mobility...they are disproportionately affected by climate change (UN Women Watch 2011: 1).

Social frameworks have allowed for vulnerability of people to be more closely examined, bringing gendered issues to the fore of climate change discussions. The focus on 'women' as a distinct group illustrates that there is a heavy reliance on sex, i.e. 'women' and 'men,' in examining inherent power relations within society. Andrea Cornwall frames this reliance on sex as a categorising tool as follows, "What we see at work is a series of transpositions. 'Gender' becomes fixed as sexual difference. This frames two categories, ‘women' and 'men', which, like all dichotomies, are bounded and mutually exclusive" (Cornwall 2007: 73). The language that is used when talking about gendered vulnerability within climate change literature can be seen to be drawing on these categories.

Despite the assertion made by Piers Blaikie, Terry Cannon, Ian Davis and Ben Wisner that social frameworks of vulnerability allow for the impact of disasters to be assessed with an acknowledgement of different circumstances of people and the differences among them, what has been shown in the above section is that there is a strong focus on 'women' as a distinct group being portrayed as vulnerable. By consistently drawing overarching portrayals of women as vulnerable, not only are assumptions made about women as a homogeneous group but also all women are set 
up as vulnerable. This implies that therefore all men are not vulnerable and, if we take the argument further, are in fact reinforcing women's vulnerability. This categorisation of women and men based on sex can again be seen to draw on simple dualisms, as illustrated below:

Table 3: Dualisms based on sex

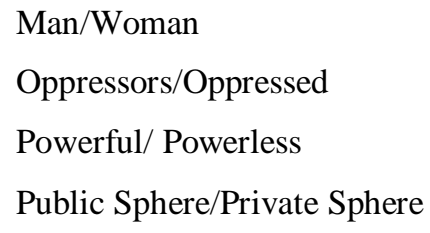

This reliance on simple dualisms, based on sex, positions all men as the powerful oppressors and all women as the powerless oppressed. Framings such as these do not reflect the true complexities of power within the climate change debate. The people of Kiribati, both men and women, face inequality on a different scale when it comes to climate change. As a country that is not only reliant on the international community to respond with effective and immediate mitigation strategies - the type of which Kiribati need not implement itself based on the negligible level of emissions that they produce-but also as an economy reliant on aid assistance, its ability to respond, or lack thereof, is of a different order.

Within black, indigenous, and 'Third World' feminist writing, criticism has been levelled at western feminists' tendency to make assumptions about universal inequalities that all women face based purely on sex (Trask 1993; Griffen, V 2006; Griffen, A 1989). This expression of universal oppression faced by women fails to take into consideration the differences between women and at the same time sets all men up as the oppressors. As Cheryl McEwan notes, "Many 'black' and 'third world' activists object to western feminism that depicts men as the primary source of oppression; gender oppression is inextricably bound up with race and class” (McEwan 2001: 98).

The assigning of such definitive categorising for articulating women's vulnerability to climate change also presents a picture of both inevitability and distance. While there is a clear attempt to provide an analysis of the social structures that lead to women being more vulnerable to climate change, the consistent reference to 'women' as a distinct group fails to take into consideration the wider political dynamics that are present in the climate change debate. Inequalities that transcend sex 
as a categorising tool can be considered, including inequality based on for example geographic location, ethnicity and class (to name a few). This then allows for vulnerability to be assessed with an awareness of the interlocking inequalities that climate change highlights.

While the literature on women and climate change almost explicitly links women and vulnerability together, within feminist writing and research there remains a debate around whether this is a constructive way of portraying women at all. Although there appears to be wide agreement that women should be included in climate change discussions, programmes and policies, on what basis women should be included remains debated. As has been demonstrated in Chapter two, literature has been produced which questions the overwhelming categorisation of women as vulnerable within the climate change debate (Nayar 2011; MacGregor 2010; Murthy 2000). The questioning of the productiveness of language that seemingly allocates only one attribute to women, that of vulnerability, has been challenged by some feminists who argue that women should be viewed as agents of change with particular strengths that they can bring to the table in relation to environmental discussions (Nayar 2010; MacGregor 2010; Blomstrom, Cunningham, Johnson \& Owren 2009). Blomstrom et al, for example argue women should be viewed as agents of change as their various roles in society allow for certain skills to be brought into the climate change debate. These images are being used to advocate the importance of women's involvement at all levels of the climate change debate based not on vulnerability but rather as change agents with specialised knowledge.

An example of this can be seen at the 2000 UNFCC Conference of the Parties (COP), where a side event was held entitled "The Power of Feminine Values in Climate Change” (Hemmati \& Rohr 2009: 22). This articulation of women implies that women have a unique value to add to the climate change debate based on some concept of femininity based on sexual difference. This construction of women as agents of change appears to be drawing on the foundations of ecofeminism, in which women are viewed as closer to nature, therefore allowing them to have specialised knowledge that men do not possess. This is articulated by Cecile Jackson, who uses Vandana Shiva's analysis of women and nature, stating, "The ecofeminist conception of the inherent closeness of women and nature is expressed by Vandana Shiva '(The) organic process of growth in which women and nature work in partnership with each other has created a special relationship of women with nature...”' (Jackson 1993: 
1947). Arguments such as these, while also drawing on conceptions of women's traditional roles, make the point that it is these very roles that position women as change agents as their engagement with the environment is conceptualised in ways that are unique from their counterparts, men.

This capacity for responsiveness is, seemingly paradoxically, aligned with the very roles that others have argued reinforces their vulnerability. Irene Dankelman also argues that women should be viewed as agents of change in an attempt to highlight a broader way of assessing women within the climate change debate. Dankelman takes the articulation of women's roles within society further and argues that these roles not only bring specific environmental knowledge but also that, because of this, women have been and continue to be active spokeswomen for the environment. Dankelman says,

Day after day they [women] perform their communities' productive and reproductive tasks, or inform the world communities about the need for environmental conservation. They sustain the interface between the human and physical environment, thereby demonstrating a deep understanding and technical knowledge about the ecological characteristics of their environment (Dankelman 2009: 3).

Using the same analysis of gendered roles within society, Dankelman makes the point that rather than inclusion of women based on vulnerability caused by their gendered roles solely, women are in fact an important resource with specialised knowledge and should also be depicted as agents of change within environmental debate. This is a argument echoed by ISIS International Women's House, an international organisation working on women's human rights and networking and information sharing of women's movements in the global south, who argue, "Climate change is the living reality of women, with two facets - on the one hand, women are indeed the most affected because of long standing issues of gender, on the other hand, they are also active agents in addressing immediate and strategic solutions to climate justice..." (ISIS 2012: 1).

When we examine these arguments from a Pacific perspective, they would seemingly hold some relevance. In research conducted in the Pacific that explored how to build gender responsive adaptation approaches to climate change, Ruth Lane and Rebeca McNaught assert, "Gendered divisions of labour in non-disaster or normal 
times inform the way and extent to which communities can adapt to extreme climatic events. Local gender-specific knowledge must be recognised; it can contribute much to furthering the existing body of knowledge on climate change, as many communities across the Pacific are already witnessing and adapting to changes that are affecting their livelihoods” (Lane \& McNaught 2009: 108). They use the example of a drought on the island of Yap in the Federated States of Micronesia, where local women used their knowledge about hydrology from working the land to find and dig a well to access drinkable water (Lane \& McNaught 2009). While this clearly demonstrates the specific knowledge that women have, generated by gendered divisions of labour, and encourages inclusion of women's knowledge, Lane and McNaught go on to highlight concerns that have been raised by Pacific women stating, “... [women] raised concerns about the fact that the bulk of decision-making in relation to resource allocation following disasters was being carried out by men. Further, there was concern that decisions made by men at the household and community level were not always fair, and most commonly did not involve women...” (Lane \& McNaught 2009: 108). What this illustrates is that while it is important to acknowledge the gendered roles within society and the specialised skills and knowledge that women have from undertaking these roles, it is these very roles that can also bind women into predefined positions. In other words, positions that although on the surface would appear to indicate a level of agency within the process can also be counterproductive and further entrench vulnerability. This does however raise some interesting questions to consider. Is a woman spokesperson considered an agent of change if she does not draw attention to women's issues? Or does the very fact that she is a woman and is speaking about the environment benefit women and help advocate their needs?

These two contrasting approaches present differing images of women, the first being that women are vulnerable based upon their position in society, and the second being that women are uniquely connected to nature based both on their roles within society and based on their biology as women. These contrasting images of women, while seemingly in contradiction with each other, both establish an analysis of women that is restrictive. As Andrea Cornwall argues, "Such representations of women often take the shape of powerful and rousing 'gender myths' in which their protagonists come to be represented as abject victims, 'the poorest of the poor, or brave heroines who battle against all odds for a better life for their children and communities"” 
(Cornwall 2007: 73). As Cornwall (2007) notes, women are cast either as victims of climate change or they are agents of change.

The attention to essentialism in feminist theory arose from critiques of essentialist notions of biological differences based on sex. However, with the growing development of feminist writing, critiques of essentialism have also focused on other writing that creates a sense of a homogenous group, whether it be based on biological difference or the impact of society. Deborah Cameron's analysis of essentialism, in which she challenges historical understandings of essentialism with feminist discourse, is illustrative:

It is important to grasp, however, that when feminists talk about essentialism they do not always — or even often, nowadays—mean the biological kind... When one feminist is labelled essentialist by another, the intended criticism is more likely that she treats women as a) in some fundamental way all the same as each other, and b) fundamentally different from men (Cameron 1998: 16).

I would contend that while both these constructions of women-as the disproportionately vulnerable and as agents of change-are intended to provide a greater awareness of the gendered implications of climate change, the basis on which these positions are being argued limits the ways in which women are being talked about. The result is that essentialist notions of women are being used in both constructions of women. This establishes a situation where women, whether being described as vulnerable based on sex or social structures, or viewed as agents of change based on some innate quality they posses as women, are framed into two distinctive categories that are both underpinned by essentialist notions of 'women'. The better analysis in my view is that women are neither intrinsically vulnerable nor intrinsically agent provocateurs. By only presenting women in one or the other of these two contradictory ways, critical analyses cannot fully respond to the complexities of the climate change debate, nor address questions regarding the roles that women currently play, and the ways they can and should be included within the debate in the future.

Thus far discourses of vulnerability have been discussed in a contextual sense, locating the terms that are being drawn upon to provide a clearer picture of what is being presented when talking about both Pacific Island and women's vulnerability. The examination of the inherent and complex power dynamics that arise with the 
reliance on such terms as vulnerability have been examined and questioned. The binaries in our understandings of discourse discussed above have highlighted the at times contradictory nature of such language within climate change debate. In order to examine the possible impacts of such language on outcomes for I-Kiribati, the next section focuses specifically on discussions of forced migration of Kiribati as an outcome of climate change.

\section{Forced Migration}

The IPCC in 1990 identified the fear that the biggest impact of climate change will be on human migration. As climate change knowledge has increased, awareness of the impacts that rising sea-levels, coastal erosion, coastal flooding and agricultural disruption will have on places like Kiribati has led to forced migration becoming a central issue. Kiribati, in particular, has been identified as one place that is going to experience severe effects from climate change. Subsequently, ideas about sinking islands that will force I-Kiribati from their homes have come to dominate the world's view and belief regarding Kiribati's expected fate. When looking at the impact of climate change on Kiribati, forced migration has become a possible outcome that has captured the world's imagination.

It is important to establish at this point that the proposition of this research is not that forced migration is an outcome based on vulnerability discourses alone. Language does not magically conjure global warming and sea level rise; however, our understanding of problems - and their solutions — is shaped in and through language, and therefore language use impacts policy outcomes. This research questions the way that vulnerability discourses that appear in discussions of climate change and Kiribati impact upon the way forced migration is presented as an option and an outcome of climate change for places such as Kiribati. This section of the research focuses on questions such as, how does consistently grounding Kiribati's vulnerability in an economic paradigm influence the consideration of mitigation and adaptation strategies? Does an economic paradigm strengthen the view that, for Kiribati, forced migration is inevitable? It then discusses models of migration more broadly, highlighting the disproportionate attention that the scenario of forced migration receives when compared with existing migration. And it looks at the complex roles of choice and agency in relation to migration. 
In order to examine the role that vulnerability discourse plays in forced migration as an outcome for Kiribati, it is first necessary to establish specific understandings of forced migration in the context of climate change. Researchers who have explored migration factors, and in particular migration due to climate change, have noted the ambiguous nature of the causes of migration. Dr Camillo Boano, Professor Roger Zetter and Dr Tim Morris, writing for the Refugee Studies Centre, note, "Migration, and population movement in general, is part of human history and an important adaptive mechanism. Thus, it has always been difficult to differentiate 'environmental refugees’ from ‘economic migrants'” (Boano, Zetter \& Morris 2008: 5). Historically the most common theoretical concept used to understand migration patterns is the push/pull model. This model presents an uncomplicated image of simple 'pull' factors that are largely based on economics, such as the lure of economic opportunities thought to draw, or pull, migrants from developing countries to developed countries. Conversely, 'push' factors, based on economics and/or social and political hardships in the sending countries, are thought to compel or 'push' migrants to leave their country of origin (European Commission 2000).

However, this model for interpreting migration patterns only provides a simplistic notion of causes for migration; migration is in actuality far more complex than this model can account for, particularly when environmental factors are explored. Castels, for example, notes that migration is created by "Complex patterns of multiple causality, in which natural and environmental factors are closely linked to economic, social, and political ones” (Castels 2002: 25). The multiple causes of migration have led many to acknowledge that making assertions of specific single causes of migration is unrealistic. This has meant that there has been a reluctance to specifically focus on environmental factors as the sole cause of migration. However it is acknowledged that the environmental degradation that climate change is predicted to create will increasingly cause people to leave their homes. As Oli Brown stipulates, "Deciding causality between economic 'pull' and environmental 'push' is often highly subjective... [and] disaggregating the role of climate change from other environmental, economic, and social factors requires an ambitious analytical step into the dark” (Brown 2008: 12). Statements such as these highlight the multifaceted nature of both migration, generally, and forced migration due to climate change, specifically.

Although forced migration attributed to climate change has captured the world's 
attention, some scholars such as John Campbell have highlighted the fact that climaterelated population movement is happening already. Although not falling under the technical category of forced migration, such movements bring into focus the role that environmental degradation has on the choices to migrate. Campbell argues that migration in response to climate change falls into two distinct categories,

The first category is migration from communities adversely affected, but not affected to the extent that continued human habitation is rendered impossible. This may be considered climate induced migration...the second category is migration from communities where continued habitation becomes impossible. Such a situation would require entire communities to relocate. In this case, the migration may be considered forced, rather than induced... (Campbell in Burson 2010: 32-33).

The application of Campbell's models introduces ideas of agency: forced migration can be argued to take away agency whilst climate-induced migration can be seen as an adaptation strategy, which requires a level of agency ${ }^{1}$.

When we look at Kiribati specifically, it appears that climate-induced migration is already prevalent from Kiribati's outer islands to South Tarawa. Migration within these islands is not a new phenomenon; documentation highlights that migration between these islands has occurred for generations (Kiribati National Statistics Office 2005; Brown, O 2008; Asian Development Bank 2011). However when we look at internal migration patterns, it becomes clear that there has been a steady increase of migration from outer islands to the main island of South Tarawa. Despite the lack of absolute clarity over the causes for migration, it is largely agreed that environmental factors are playing a large part in the migration of I-Kiribati from outer islands where subsistence living is becoming increasingly jeopardised due to the impacts of climate change. Speaking about Kiribati, Justin Locke from the United Nations Development Programme argues that "Economic migratory pull factors associated with uneven development are combining with environmental push factors, forcing citizens to migrate from the outer to central islands” (Locke 2008: 173). This argument is borne out in the statistics that have been gathered by numerous organisations sources

\footnotetext{
${ }^{1}$ For related reading, see D N Collins' thesis Forced Migration and Resettlement in the Pacific: Development of a Model Addressing the Resettlement of Forced Migrants in the Pacific Islands Region from Analysis of the Banaban and Bikinian Cases (2009).
} 
including the World Bank, The Asian Development Bank and Kiribati National Statistics Office, all of which demonstrate a dramatic increase in inter-island migration. The Kiribati National Statistics Office provides the most salient statistics that show that, in 1947, only 1,671 people lived on South Tarawa, but by 2005 the official figure had risen to 40,311, and by early 2008, the unofficial figure was almost 65,000 (Kiribati National Statistics Office 2005). Despite a refusal to blame environmental degradation exclusively, the current migration patterns in Kiribati appear to adhere to Campbell's model of climate-induced migration.

Although there is an increase in migration, based on a mix of reasons, forced migration remains the image that has captured the world's attention. Referencing Kiribati's vulnerability to climate change and the threat of forced migration has become a way of demonstrating globally the severe effects of climate change in the hope of creating changed behaviours by the international community. As referenced in the earlier review of literature, academics such as John Campbell and Carol Farbotko have challenged the way in which forced migration has become the expression of vulnerability of places such as Kiribati and Tuvalu through images of 'sinking islands'. In John Campbell's article on migration and the Pacific, he notes the fact that "Environmentalist organisations have adopted small island states, in particular atoll countries, to illustrate the urgency of mitigating the problem of climate change by reducing green house gas emissions” (Campbell 2010: 22). The propensity of the international community to articulate Kiribati's vulnerability through images of sinking islands creates a far more dramatic view-one that is being used to capture the world's attention as a demonstration of what the outcomes of climate change may be. As has already been noted, the imagery of vulnerability, based on forced migration, is not exclusively being utilised by the international community. These images of Kiribati's vulnerability to seawater inundation, forcing the mass migration of I-Kiribati, are also being utilised by both the Kiribati government and I-Kiribati to some effect.

Unpacking Campbell's models of migration further it becomes clear that there is a second layer of analysis that is required to understand the differences he draws between forced migration and climate-induced migration. Such distinctions necessitate a careful consideration of the question of choice. As has been shown, migration between outer islands and South Tarawa is already increasing; however this type of population movement has been categorised as climate-induced migration, 
which as has been discussed implies a level of agency. If Campbell's model is to be applied then the movement from outer islands to South Tarawa does not fall under the category of forced migration, as currently these outer islands have not been deemed unliveable, therefore the pivotal difference in these two categories of migration is based on whether there is choice. Critical analysis of the literature on forced migration has noted the long history of Pacific migration. Epeli Hau'ofa, in his critical work critiquing the view of the Pacific that has been created through colonisation, highlights the fact that the people of the Pacific have been travelling for centuries and with huge benefits, such as creating economies, social connections and exploring previously uninhabited islands. The view that Hau'ofa presents counters that of a vulnerable island people being forced to migrate; instead he presents an image of Pacific people as brave adventurers who have carved out their own destiny. This is most evident when he claims,

This was the kind of world that bred men and women with skills and courage that took them into the unknown, to discover and populate all the habitable islands east of the $130^{\text {th }}$ meridian. The great fame that they have earned posthumously may have been romanticised, but it is solidly based on real feats that could have been performed only by those born and raised with an open sea as their home (Hau'ofa 2008: 34).

Hau'ofa highlights the fact that migration as an option for Pacific people is not necessarily negative, and instead may be a more normalised feature of their history and possible future. However, the type of migration idealised in Hau'ofa's vision is entirely one in which choice is exercised. In fact, Hau'ofa has been critiqued by colleagues for painting too romantic a picture of the region that fails to fully account for the economic, political, social and environmental factors that constrain choice (Waddell Naidu \& Hau'ofa 1993). How does Hau'ofa’s important alternative vision square with the force in the forced migration scenario? When we examine the context of migration and climate change in Kiribati, choice becomes a rather ambiguous notion.

Looking at issues of choice also becomes important when we examine Kiribati's proposed responses to forced migration. President Tong has stated on numerous occasions that ideally the Kiribati government would like a location to be found that would enable the total migration of Kiribati. However, noting the difficulty in 
achieving this, the Kiribati government has begun to introduce the concept of migration possibly taking place in the form of increased labour migration to places such as New Zealand and Australia. This would be done through job training so that migrants could seek skilled employment (Risse 2009). This concept is intended to work on two levels: first, that it will reduce the population burden that is currently being felt in Kiribati, particularly on South Tarawa, and second, it would mean the establishment of pockets of I-Kiribati communities in other countries, which could assist with later migration. This notion of preparing for future migration carries with it a strong message of inevitability. Proposals such as these indicate an element of choice, with incentives to migrate; however do proposals such as these alleviate the degree of vulnerability and do they present a real choice for I-Kiribati?

Looking at I-Kiribati women's roles within the labour market, questions also need to be asked about how skilled labour migration may further entrench I-Kiribati women's vulnerability. While it is true that Kiribati has a long history of migration, and that women have not been excluded from this migration, as has been noted in Chapter two, researchers looking at the impact of forced migration on women have commented that migration as a response to climate change could exacerbate women's vulnerability as it is often those with social capital who have the luxury of choice in migration. This group does not usually include women, due to the fact that they are restricted in their participation within the labour force by gendered roles (Haigh \& Vallely 2010; Boncour \& Burson 2010). Publishing in 1993, Taboneao Ngaebi, Tekarei Russell and Fenua Tamuera highlighted the traditional status of I-Kiribati women to account for the limited participation of women in the labour market. Noting that while I-Kiribati women were increasingly highly educated and had proven themselves in the workforce, because of traditional views of women as holding a lower status and the gendered expectations of work, many I-Kiribati women remained engaged in unpaid employment. Although not overtly discriminated against, they continued to be excluded from skilled work (Ngaebi, Russell \& Tamuera 1993). More recently, the UNESCO Country report on Kiribati lends weight to the concerns expressed about women's ability to migrate through skilled migration indicating that, "In the workforce there has been increased participation of women with an increasing number of women in professional and senior professional roles within Government, though for the most part women's employment remains in non-cash employment" (UNESCO 2009: 9). This leads to the conclusion that President Tong's oft-repeated 
objective of 'migration with dignity' through proposed increases in skilled migration would appear to leave I-Kiribati women at a distinct disadvantage, and that the choice to migrate may be one which many women are excluded from.

The previous discussion has highlighted that forced migration is very much a part of the climate change debate, both in the international arena and for the Kiribati government. However, has the focus on migration, whether it be through force or choice, created an environment were other mitigation and adaptation options have come to be ignored? Thus far this research has explored the definitions of migration that are being brought to bear within the debate. Through this research it has become apparent that despite the fact that migration is happening already, this migration has not captured the world's attention in the same way as images of 'sinking islands' and imperilled 'climate refugees.' Due to the fact that the total forced migration of Kiribati takes such a hold on the international community, and looms large as an issue for Kiribati, the following discussion will examine the impacts of vulnerability discourse on the view that forced migration is inevitable for Kiribati.

Despite the fact that Kiribati is currently implementing adaptation strategies in an attempt to minimise the negative outcomes of climate change, forced migration remains as a final adaptation option for Kiribati. Questions have already been raised in earlier chapters about the widespread notion that forced migration as a final outcome of climate change is inevitable, at the risk of other adaptation options being ignored (Asian Development Bank 2011). When we look at this argument in relation to vulnerability and adaptation, it is clear that both notions co-exist uneasily within the climate change debate. This is apparent when we look at the IPCC's definitional constructs of both adaptation and vulnerability in the context of climate change. The third Assessment Report defines vulnerability as the degree to which a system is going to feel the effect of even a moderate change in climate coupled with the adaptive capacity of that system. Adaptation or the adaptive capacity of a system is defined by the system's ability to offset the potential negative impacts that a change in climate is expected to bring (IPCC 2001). The report goes on to state that, "Under this framework a highly vulnerable system would be a system that is very sensitive to modest changes in climate, where the sensitivity includes the potential for substantive harmful effects, and for which the ability to adapt is severely constrained” (IPCC 2001: 1.4.1.). These definitional constructions of both vulnerability and adaptation capacity make it apparent that Kiribati’s classification as a highly vulnerable system 
places the ability for adaptation as a virtually unachievable goal. It can be argued this allows forced migration to be seen as possibly the only solution left open to I-Kiribati.

One of the key factors in reducing the risk of forced migration for Kiribati is that more than just adaptation strategies are required. The international scientific community has reached an almost unanimous declaration that rising temperature caused by carbon emissions is causing sea levels to rise. This is placing low-lying states at particular risk and increasing the likelihood of forced migration. In order to address this, mitigating strategies need to be put into place to achieve a lowering of carbon emissions. To explore further ideas of the inevitable nature of forced migration, I will examine the international agreement that was reached at the 2010 Cancun Summit on Climate Change.

At the 2010 Cancun Summit, at which governments and scientists met to try to reach internationally agreed upon strategies to limit the impacts of climate change, the international community agreed to "Peak emissions and an overall 2 degree target to limit temperature rise” (Department of Energy and Climate Change 2010: 1). This commitment to limit temperature rise has come under criticism by many for not being radical enough to ensure the safety of many people and places. For example, Oxfam asserts, "Two degrees is the target upon which more than 100 governments are basing their strategies because the rich world has deemed this could be an economically acceptable one. However, even warming of 2 degrees entails a devastating future for at least 660 million people” (Oxfam 2010: 5). Pacific nations have echoed this concern, asserting that a temperature rise of even this level will have devastating effects for their low-lying islands, and are instead arguing for global warming to be limited to no more than 1.5 degrees. Speaking about the commitment made to 2 degrees, Samoa's executive director of the environment organisation $\mathrm{O}$ Le Siosiomaga, Fiu Mataese Elisara, reflected, "Sadly, we have now given legitimacy to the Copenhagen Accord which will amount to 3 to 5-degree increase in temperatures, despite the science calling for a 1.5-degree Celsius increase if we are to survive as small islands countries in the Pacific” (Maclellan 2010: 1). Nic Maclellan, reporting on the Pacific Islands' calls for an 1.5 degree increase, affirms, "This target is the stated position of more than 100 nations, especially Small Islands Developing States (SIDS) and Least Developed Countries (LDCs), which are seeking reductions in global emissions to ensure that increases in global average temperature are held below 
a level that will trigger even more adverse environmental effects” (Maclellan 2010: 1).

Kevin Anderson, of the UK Tyndall Centre for Climate Change Research, critiques the reluctance of the international community to implement more dramatic goals to reduce global warming. In an article published in January 2012 by the UK Royal Society, Anderson states: "Despite the evident logic for revising the 2 degree Celsius threshold, there is little political appetite and limited academic support for such a revision. Put bluntly, while the rhetoric of policy is to reduce emissions in line with avoiding dangerous climate change, most policy advice is to accept a high probability of extremely dangerous climate change rather than propose radical and immediate emission reductions” (Anderson cited in Maclellan 2010: 1). This apparent refusal by the international community to commit to anything below a 2 degree Celsius increase gives weight to arguments mounted by scholars such as Barnett, Campbell, and Farbotko, who have questioned the way in which vulnerability discourses have played a role in creating an environment in which international inaction, justified on the basis of the inherent vulnerability and the economic status of Pacific Islands, leads to the belief that forced migration is the ultimate and inevitable outcome for places such as Kiribati.

\section{Conclusion}

Within the climate change debate it is largely agreed that low-lying islands are particularly vulnerable to the impacts of climate change. Because of the expected impact that climate change will have on Kiribati, the image of forced migration as the ultimate consequence is being promoted by both the international community and by Kiribati itself as a way of encouraging action. The images of island nations being drowned and I-Kiribati being forced from their homes simultaneously promote action whilst reinforcing images of Kiribati's vulnerability. Although these images are employed to encourage action, a perhaps unintended consequence is that they appear to be excluding alternative pictures of adaptation and resilience within the Pacific. Through the promotion of forced migration as the final, and only possible, consequence of both climate change and Kiribati's (and the wider Pacific's) vulnerability, ideas that present a counter image are being sidelined. What has become evident is that views of Pacific Islands' isolation, smallness and dependency, which amount to vulnerability, are all being reinforced by the way climate change is 
debated. This has the consequence of crowding out characterisations of the Pacific that promote agency, strength and resilience. In the same fashion, when we look at the literature on women's vulnerability, although there is an attempt to acknowledge women's skills and agency within the climate change debates, these are based on assumed gendered traits that have the effect of further constraining women to their perceived nature. Pacific countries and women are both described as vulnerable. This joins them in a state of dependence, with diminished agency and subject to an inevitable outcome. 


\section{Chapter Five: Articulations of Kiribati's and I-Kiribati Women's Vulnerability by Pelenise Alofa and Maria Tiimon}

Previous chapters have demonstrated that Kiribati has become, for many commentators, a powerful example of the possible consequences that a continuing changing climate could bring. Scientific evaluations disseminated by the IPCC have categorised Kiribati as one of the countries most vulnerable to climate change, while international agencies lobbying for action on climate change have used Kiribati's situation to demonstrate the urgency of action that is required. Throughout the vast amounts of research published about Kiribati and the calls for action for Kiribati, discourses of vulnerability are utilised continually. Kiribati's smallness, isolation, low-lying geography and position as a less developed country have all been highlighted to articulate and reinforce these ideas of Kiribati's vulnerability. Descriptive language and images of Kiribati being swallowed by rising sea levels, coupled with Kiribati's limited ability to respond to such impacts, have led to predictions of forced migration. These images have been utilised to demonstrate the impacts that not responding to change will have. However, as has been illustrated, many Pacific scholars have disputed the ways that Pacific Islands have been articulated within international contexts and offered different contextual boundaries for viewing and analysing the Pacific region.

This chapter explores how I-Kiribati advocate for themselves and are articulating both Kiribati's and I-Kiribati women's situations in relation to climate change. In order to provide a clear sense of how I-Kiribati people are articulating their own position within climate change discussions, this chapter will focus on two individuals who are directly involved in Kiribati affairs and climate action for Kiribati. These are Maria Tiimon, who currently works for the Pacific Calling Partnership within the Edmond Rice Centre in Australia, and Pelenise Alofa, from Kiribati Climate Action Network or KIRICAN. The choice to focus on these two particular actors is because both women have been published extensively and their interviews and work are within the public domain. Throughout this chapter and elsewhere in the thesis both Alofa and Tiimon are referred to as agents of change. The choice to depict Alofa and Tiimon in this way is based on Nayar's expression of women as change agents in 
which she argues, "We need to recognise women as change agents engaged in the struggles over fossil fuel, exploitation, pollution and environmental health” (Nayar 2011: 1). With the growing interest in Kiribati and climate change, these two women have received a substantial amount of attention and remain engaged in the debates through their actions. While Nayar provides the basis for Alofa and Tiimon's depiction of 'agents of change,' there is also an awareness of the warning that Rohr provides in challenging the shift towards viewing women as agents of change. Rohr notes that in viewing women as agents of change it shifts the responsibility of action onto individuals' shoulders who often have little impact on political decisions (Rohr 2011). This is a salient point to consider. While this thesis contends that both Alofa and Tiimon are agents of change through their activism, it recognises that it is not the responsibility of Alofa and Tiimon to effect significant change on an issue that requires a global solution.

The following sections will explore how both Alofa and Tiimon are articulating the situation of Kiribati and I-Kiribati in relation to climate change. These sections examine whether the language that these I-Kiribati spokespeople are using creates different or similar pictures of Kiribati's vulnerability within the climate change context. This chapter starts by revisiting the theoretical debates about how the Pacific and specifically Kiribati is framed. The purpose of providing this brief overview is to provide grounding for analyses of Alofa and Tiimon's articulations of Kiribati. The first section draws on Hau'ofa's argument that the way the Pacific is framed by those outside the region impacts how those from within view themselves. Barnett and Campbell's argument is then reintroduced in which they claim that terms such as vulnerable have been appropriated by those from within the Pacific in order to carry their messages of action to the international stage. These two positions offer contrasting views about Pacific people's uptake of the language that is used to describe the Pacific by outsiders, and provide an interesting counterpoint for analysis of both Alofa's and Tiimon's articulations of Kiribati.

This is followed by the introduction of Alofa's and Tiimon's voices. The progression of this section of the chapter follows the debate through discussions of mitigation and adaptation policy responses. This progression echoes the progression that the international community has followed, with first a consideration of mitigation and then adaptation. The focus on first mitigation and then adaptation allows for a concise analysis of how Alofa and Tiimon are drawing on biophysical and social 
frameworks of vulnerability. It also allows for the interrogation of how Alofa and Tiimon are drawing on these frameworks and what this means for the way that they both articulate Kiribati.

As demonstrated in the previous chapter, the views about how best to categorise women within the climate change debate are contentious. Issues of agency and vulnerability have been academically contested in relation to the power relationships that the terminology of vulnerability creates and sustains. For I-Kiribati women, it is these debates that are shaping the way that they are included in discussions of climate change. The focus of this research so far has been on how women have been brought into climate change conversations by those from outside the Pacific. It is therefore imperative to examine the ways in which I-Kiribati women are being contextualised, and whether they are being explicitly referenced in discussions at all, from actors within Kiribati society.

The final section of this chapter explores the way that Alofa and Tiimon are bringing (or not bringing) I-Kiribati women into their discussions. Their references to women are then analysed from within the broader debate about women as vulnerable versus women as agents of change and is followed by suggested explanations of why these two women may be choosing to include or not include I-Kiribati women in their discussions.

\section{Framing the Pacific: Recap of the Theoretical Debate}

The climate change debate has seen those outside the Pacific consistently draw on terms such as small, isolated and developing to indicate vulnerability of places within the Pacific such as Kiribati. Although these terms have been used in assessing biophysical and social frameworks of vulnerability, Chapter four has argued that the impact of such language has meant that rather than vulnerability being assessed as a consequence of climate change, vulnerability is instead being framed as an innate quality, which climate change is seen as exacerbating.

Chapter four presented the arguments made by Briguglio, Pelling and Uttio in which the Pacific's vulnerability is an inherent factor based upon its limited economic size and remoteness from larger economic markets. These assessments of the Pacific's vulnerability present an image of the Pacific as innately vulnerable with climate change being the factor that has highlighted this vulnerability. A quotation in an article published by Phil Mercer is a perfect example of the way in which 
smallness, isolation and Pacific Island economies have been used to account for their vulnerability. Mercer quotes the Australian Parliamentary Secretary for International Development, Bob McMullan, who argues, "People often fail to understand the depth of the challenges which the governments of many Pacific island countries face when you look at the... location and the scale of the operation they have to do in providing basic services to a very dispersed population across great distances with few resources” (Mercer, P. 2009: 1). Mercer's article can be seen to be drawing on the binaries that were presented in Chapter four which view the Pacific as remote compared to a centre outside of the Pacific. In this statement Mercer also highlights the view of the Pacific as small and scattered. This quote points to the view that not only are islands within the Pacific isolated from bigger economic zones, they are also isolated from each other, and it is these factors that are seen as fundamentally hindering any economic growth. This chapter is focused on examining the language used by Alofa and Tiimon and is asking questions such as, are Alofa and Tiimon drawing on the same or similar language to describe Kiribati and if so how does this affect the way in which the debate is framed by them?

In asking these questions it is pertinent to again draw on Pacific scholar Epeli Hau'ofa's critique of the way the Pacific has been framed, with particular focus on how this affects those that are being spoken about in such ways. This thesis has thus far drawn heavily from Hau'ofa who has critiqued the framing of the Pacific in this way and argued that not only is it an inaccurate view of the Pacific, but also belittling. Hau'ofa argues that the consistent use of such language that presents the Pacific as small, isolated and therefore dependent can also become embedded in the consciousness of those spoken about in this way. Hau'ofa argues

Views held by those in dominant positions about their subordinates can have significant consequences for people’s self image and for the ways they cope with their situations. Such views, often derogatory and belittling, are integral to most relationships of dominance and subordination, wherein superiors behave in ways or say things that are accepted by their inferiors, who in turn behave in ways that perpetuate that relationship (Hau'ofa 2008: 28).

This is an important argument to consider in relation to the climate change debate. As has already been shown, references to Kiribati routinely invoke adjectives such as small, isolated and low-lying in order to ground biophysical and social 
understandings of vulnerability. This thesis contends that these terms frame places such as Kiribati as innately vulnerable, and allows for responsibility for action to be excluded from the debate. This chapter will be drawing on Hau'ofa's arguments as a way of assessing how Alofa and Tiimon articulate Kiribati: does their use of language present an image of the Pacific similar to Hau'ofa's, or does it instead support Hau'ofa's argument regarding the internalisation of belittling discourse?

So far this thesis has examined the language that has been used to label the Pacific from outside. Chapter three demonstrated that terminology such as small, isolated and developing have a long history in relation to how the Pacific is viewed by those outside, and that by viewing the Pacific through these lenses, forced migration has come to be understood as an inevitable outcome for places such as Kiribati based on understandings of their innate vulnerability. However, as Barnett and Campbell note, this language has also been used by those from within the Pacific as a way of gaining attention for the Pacific's particular issues due to climate change. Barnett and Campbell describe the language that is being used when talking about the Pacific as a “discourse of danger” (2010). These so-called discourses of danger can be seen throughout the climate change debate and literature, as evidenced throughout this thesis.

Chapter three highlighted the way in which constructions of smallness have been established, indicating that for those outside of the Pacific, vulnerability is an innate quality created by small size. These concepts are largely based on ideas of economic significance as opposed to actual landmass. Pacific leaders have also used adjectives such as these, despite the negative connotations attached to them by those from outside the Pacific, to describe island states. As Barnett and Campbell note, "All independent island countries in the Pacific region identify themselves as small island developing states (SIDS)” (Barnett \& Campbell 2010: 155-156). However, the construction of these terms when self-identified carries significantly different meanings. Barnett and Campbell argue that this definitional construction of smallness is based on no more than the simple fact that islands by definition are smaller landmasses than continents. When this understanding of smallness is applied it is then a reasonable argument to make that geographical smallness does not necessarily equal vulnerability. This analysis of smallness is in opposition to discourses of smallness applied by those outside the Pacific, in which smallness is based around the smallness of economic markets and is seen as making islands such as Kiribati intrinsically 
vulnerable. These two different definitional constructions of smallness are important to bear in mind when examining the language that is being used by those from within the Pacific to articulate their position within the climate change debate. Definitional constructions of these adjectives are particularly important when we examine concepts of biophysical vulnerability and social frameworks of vulnerability.

The literature review on the evolution of the climate change debate illustrated that there are two main focuses, the first being that of mitigation, with a clear focus on emissions cuts to stem the tide of climate change. Mitigation action calls for biophysical understandings of vulnerability to be expressed. For Kiribati it is obvious that increases in emissions will continue to see sea levels rise, which places Kiribati in a distinctly vulnerable position due to its low-lying geography. The second strand of climate change debate has focused on adaptation. Adaptation strategies are largely based on the fundamental premise that climate change is happening and that despite best efforts to reduce emissions, there will still be consequences to which countries will need to adapt. It is apparent that the vulnerability discourses that are being used to assess a country's ability to adapt are adhering to social frameworks of vulnerability. As the literature review noted, over time there has been a shift of focus by the international community to issues of adaptation alongside mitigation. However the language that is used to identify Kiribati's vulnerability has seemingly stayed the same.

As noted in Chapter four, there has been a call for qualifying adjectives to be used so as to identify differences between biophysical and social frameworks of vulnerability. However, as Chapter four illustrated, the predominance of these adjectives — rather than make more explicit the distinctions between biophysical and social vulnerability-has in fact further blurred varying understandings of vulnerability, as it appears that when these terms are being applied they are in fact all referencing the Pacific's small economic geographies. This framing of the Pacific has led to vulnerability being viewed as an innate quality as opposed to vulnerability being caused by climate change.

In turning to look at Alofa and Tiimon this chapter will follow the progression of the climate change debate from mitigation to adaptation. Ordering this chapter in this way provides a clear analysis of how Alofa and Tiimon are defining Kiribati and how this influences the way that the climate change debate is framed. In order to provide a coherent and in-depth analysis of their publications and public commentary, this 
research will focus on statements made between 2009 until 2011. These dates include key climate change conferences, starting with the 2009 COP15 conference held in Copenhagen, and concluding with COP17 in Durban. It is through these international events that both of these individuals have gained publicity in regard to climate change issues.

\section{Calls for Mitigation}

In a campaign labelled '100 days till Copenhagen' produced in 2009, Alofa talks about rising sea levels causing salty drinking water. When appealing to, in this case, the New Zealand government to take a clear stand on emission cuts, Alofa states,

New Zealand is not taking our problem with urgency and seriously, that is why we keep asking, cut the emissions so that we can do something, ten to twenty percent cuts NO that is too small, we are asking forty to fifty by $2020 \ldots$ its not too late to do something about climate change... (Alofa in "100 days to..." 2009.1: 35-2: 13).

The presentation of this message makes it appear that, for Alofa, mitigation is the pressing issue and that with a decrease in emissions this will prevent disaster for her country. This is a sentiment repeated by Alofa when she is interviewed from Copenhagen. She argues,

My problem is those that caused the problem are not trying to help, that's my big problem because if they help right now the water will stop rising, see, so my big problem is that they are not listening when we are telling them please stop it now. Stop the carbon emissions and help us so that we will save our islands and the water stops coming up. If they stop the carbon emissions and at the same time, they can help us to build our sea walls and to get fresh water then we solve the problem we don't have to move...(Alofa in "Paradise in Kiribati” 0:04-0:42).

Both of Alofa's statements make it apparent that at the time of the Copenhagen Climate Change Conference, it was mitigation that was the pressing issue for both Alofa and Kiribati. These statements also make it obvious that at this time there was a feeling that mitigation will prevent the imminent disaster that has been forecast for Kiribati. Although Alofa does not mention Kiribati’s vulnerability, the way in which 
her statements request help from New Zealand conveys a level of unspoken vulnerability.

Turning to look at Tiimon and the statements that she is making around the same time, there appears to be symmetry to what both these women are expressing as the key concerns for Kiribati in relation to mitigation. Tiimon, in a video made in coordination with the Edmond Rice Centre in Australia (which is tasked with spreading awareness about climate change within Australia), can be seen to be expressing the same focus on mitigation strategies as a way of helping Kiribati. Tiimon pleads, "So on behalf of these vulnerable people please Australia we need your help by cutting your emissions...” (Tiimon in "Climate change: Pacific Calling” 4:19-4:32). While Tiimon, like Alofa, is calling for help from those outside the Pacific Islands to make emission cuts, Tiimon is explicitly using the term vulnerable not as a way of describing the situation but rather as a way of describing I-Kiribati people. Within the context of her entire speech Tiimon is clearly drawing on what Barnett and Campbell position as a discourse of danger, with the discussions of outcomes such as forced migration being presented in a way that implies inevitability. It is in these early statements calling for mitigation responses to climate change where adjectives for describing the Pacific such as small, low-lying and isolated are drawn upon.

\section{The Application of Biophysical Vulnerability Frameworks}

What becomes apparent through the examination of articulations about Kiribati is that adjectives such as small are present in both the language that is used when international organisations talk about Kiribati but also in the way that I-Kiribati people such as Alofa and Tiimon describe Kiribati within the climate change debate. When describing the impact that rising sea levels will have, both Alofa and Tiimon have at times used descriptive terms such as small and low-lying to indicate why Kiribati is so vulnerable to these continued changes unless there is a commitment to emission cuts. For example, when describing her home land of Kiribati, Alofa comments, "It is just a flat atoll, the highest point is just say four metres above sea level” (Alofa in “100 days to... 0:09-0:14). This reference to Kiribati’s low-lying geography is being used as a way of locating Kiribati within its geographical context while also highlighting the risk that a rise in sea level would have. Vulnerability is stated based on these geographical facts and on the information that is available around how climate change will affect the environment. 
This is a sentiment that is echoed in Maria Tiimon's statements about Kiribati. For example Tiimon explains,

Kiribati is one of the smallest and most low-lying island nations in the Pacific. It is made up of thirty three islands, twenty one of them inhabited, and most being only two to three metres above sealevel... Our small country is facing critically difficult times due to climate change on our culture and our future... (Tiimon 2011: 25).

Both Tiimon's and Alofa's statements indicate that when comments on Kiribati's smallness, isolation and low-lying atolls are brought into the conversation by IKiribati to demonstrate their vulnerability, it is biophysical vulnerability in its truest sense that they are talking about. The projected outcomes of sea level rise mean it is imperative for I-Kiribati to express the low-lying nature of their islands particularly when arguing for emission cuts, which are seen to limit the rate at which the sea is rising. It becomes apparent in the above discussions that when both Alofa and Tiimon are making use of terms such as small there is a clear link to biophysical vulnerability frameworks.

However, the use of these terms has been influenced by images of the Pacific presented by those from outside, and such terms sit in contradiction to the way that scholars such as Hau'ofa have presented the Pacific. The way in which these terms are being used reinforces long held beliefs about the Pacific. Both Alofa and Tiimon make consistent reference to Kiribati's small size in relation to concepts of space restricted to land mass, which, it could be argued, demonstrates Hau'ofa's argument of internalisation. However Hau'ofa presents oppositional framings of the Pacific, in which the Pacific is viewed as either innately small and vulnerable or large and selfsufficient. In the context of the climate change debate, this thesis contends that the truth lies somewhere in the middle and that a more fluid understanding of the framing of the Pacific is required.

\section{Appropriation of Language}

As has been shown in earlier chapters, references to Kiribati's size have been used as a way of highlighting the vulnerability of places such as Kiribati from those both outside of Kiribati and from within. Barnett and Campbell have argued that the appropriation of vulnerability discourse within climate change debate by those from 
the Pacific has been used as a tool to draw attention to the position that places like Kiribati are in. Barnett and Campbell make the argument that,

The appropriation of vulnerability discourse by people from the region should be understood as both a reflection of what they understand of the serious risks they face and the language in which that situation is commonly described, and as a strategy to use the language of the powerful to draw attention to the need to reduce emissions and support adaptation (Barnett \& Campbell 2010: 166).

Examining the language that both Alofa and Tiimon use, it becomes apparent that this is a strategy that they have adopted. Although both women are using adjectives such as small to identify Kiribati's biophysical vulnerability, they are also using terms such as small to reinforce their pleas for action. This is no more evident than in this statement made by Tiimon,

[Kiribati] is a small nation of 100,000 peoples scattered across 33 coral atolls in the central Pacific Ocean. Our 33 tiny coral atolls are flat and no more than two metres above the sea level. Any increase in sea level will have disastrous effects on our country (Tiimon in “Climate Change: Pacific calling” 2009: 0:39-1:03).

While Tiimon is applying the term small to describe Kiribati's geographical contours, she takes this further by including the descriptive term 'tiny' to further illustrate the impacts that climate change could have. The use of this type of language is clearly emotive. Although the term vulnerability is not directly mentioned, this type of language reinforces ideas of vulnerability by making explicit the risk that Kiribati as a 'tiny' island nation faces due to rising sea levels caused by climate change. Tiimon's statement is a good example of what Barnett and Campbell argue is the appropriation of vulnerability discourse within climate change debate by those from the Pacific, and is repeated by both women alongside calls for action.

Alofa and Tiimon, in their personal speeches and when speaking on behalf of campaigns, have echoed vulnerability discourse in their bid for action. This can be seen in a speech delivered at COP15 in which Alofa urges, "I am here asking all world leaders to ACT NOW before it is too late to save my countries Kiribati and Tuvalu. We need leaders who recognise the moral obligation to hear the voices of the most vulnerable...” (Pacific Calling Partnership 2009: 1). This statement is clearly 
drawing on the discourse of danger that has been so pervasive throughout the climate change debate in regard to Kiribati.

This is also a method used by Tiimon through the use of video clips that she presented at the COP15 at Copenhagen. Tiimon begins by asserting, "We I-Kiribati respect our elders. They are the ones who give us words of wisdom and I would like you to listen to what they are saying” (Finnane 2010: 5-6) Tiimon then uses her elders as a way of conveying her message. "We are pleading to that meeting in Copenhagen that they take a look at the state of our country. The rising sea level and the heat of the sun have affected us I-Kiribati. Again we plead to the bigger countries that they help us. I thank you” (Finnane 2010: 5-6). The reference to 'bigger countries' establishes Kiribati as a smaller country making a plea for help. Statements such as these provide real depth to Hau'ofa's argument about the danger of language in relation to the Pacific, and are seen to be repeated by Alofa. Alofa makes the statement, "We are hoping that the bigger industrialised nations, who make the big decisions will be fair to us..." (Thaysen 2009: 1). This statement not only indicates Kiribati’s small size by positioning industrialised countries as 'bigger' but also draws attention to the decision-making power that these bigger countries have to make the 'big decisions'.

Turning to look at social vulnerability frameworks, which are intended to be used to assess the social structures that allow for a country to respond to climate change, illustrates the complexities of the way in which the Pacific's vulnerability is framed. Looking at both Alofa and Tiimon, it becomes clear that both of these women's messages have also shifted from one in which emissions cuts are the main focus to a call for adaptation. Investigating the language that surrounds adaptation policies and vulnerability, what becomes apparent is that social frameworks of vulnerability are most often being applied. It is through the application of social vulnerability understandings that Kiribati's economic situation is again being brought into the debate, with its perceived ability to respond to climate change being questioned.

\section{Shift to Adaptation}

Through the development of climate science there appears to be a gathering consensus by both the scientific and political community that mitigation responses alone will not fix climate change and that adaptation is a key response that is needed. Both Alofa and Tiimon can be seen to have followed this shift in climate change 
knowledge and have altered their calls for action to include climate finance for adaptation. Although earlier statements indicate that mitigation was the pressing issue for Alofa, her awareness of the need for climate finance is evidenced as early as 2009, in the same year as the Copenhagen climate conference; when asked by an interviewer what she sees as the biggest problem for Kiribati she responds, "It's the money, we do not have the money, we do not have the technology...” (Alofa in “Climate testimonies:..." 0:09-0:24). This statement indicates that while at the time of the Copenhagen Conference it was mitigation which was at the front of her mind, there was also an awareness of the need for funding adaptation. This could be seen as pessimism about the achievability of substantial mitigation policies by the international community, or it could be seen as an acknowledgement that even with mitigation, adaptation and funding for adaptation is a necessity. This is a sentiment that Alofa has repeated since that interview. Alofa, when giving an interview from Cancun Climate talks in 2010 argues, "When we talk about climate finance we really need that not just to help our people to adapt and adjust to what is happening back home with climate change right now and [but] to help our people build the capacity for our young people to negotiate, to be able to do the ground work back home on the impacts that are happening right now..." (Alofa in "Fighting for survival..." 0:541:19). Alofa's statements highlight the urgency with which she sees climate finance as necessary for Kiribati, not only to adapt to the impacts of climate change but also to build capacity for I-Kiribati people to continue on their struggle in the international stage. The attention to climate finance is echoed by Tiimon who, when giving a speech at Sydney Palm Sunday in 2010, states, "We need an international agreement on urgent and deep mitigation and substantial funds for adaptation and technology transfer in developing countries..." (Tiimon 2010: 1). This statement is a clear departure from Tiimon's earlier calls for emission cuts alone. While emission cuts are still being demanded this is linked with urgency in funding for adaptation.

\section{Framing of Social Vulnerability by Alofa and Tiimon}

Reference to Kiribati's lack of resources has been a key factor in descriptions of vulnerability. As has been shown, the framing of Kiribati as vulnerable has been largely influenced by concepts of size and isolation in relation to economics. Both Alofa and Tiimon, however, contradict this portrayal of Kiribati while using the same language that has been used to describe the Pacific. For example, in an article entitled 
"Little or small is Beautiful!!! A small island take on the stakes in Bonn," Alofa contends "Even though our lands are small, they have given us everything in the past and the present, and we are satisfied" (Alofa 2011: 1). Alofa's use of the term small to describe her island of Kiribati creates a very different image of smallness, one in which smallness is not seen as the negative that those from outside have painted it as. This counter view of Kiribati can also be seen in an interview that Alofa gave at Cancun in 2010. When asked if Kiribati was sustainable, she responded,

Yes it is sustainable if I return to my village because I have my land, I have my house, right. Even if it is a thatch house it is a house, my basic needs are available, but the basic need is taken away from me today by climate change...That's the difference between the big countries and us, we are sustainable. I can return home and maybe in the eyes of other people I am poor but I am not poor. If I have my basic needs I have land, I have a house, I have food, I have clothing...so I feel that in my community it is sustainable but we are having problems...I do not think of economics you know as poverty... (Alofa in Climate Action Network at COP16. 17:12-18:29).

This presents a very different perspective on Kiribati, one in which Kiribati is not viewed as economically dependent or poor, but rather, due to the impacts of climate change, is a country facing changes that are affecting what has been a sustainable way of life.

Tiimon also presents Kiribati in this way, which can be seen in a speech where she outlines the way in which Kiribati culture interacts with economics, "As each family still fends for itself, things such as the best places for fishing, handicraft techniques and other skills are kept within the family. The culture of Kiribati has been preserved by the isolation that comes with being in the middle of the Pacific ocean...” (Tiimon 2010: 25). Rather than referencing isolation as a negative factor that has hindered Kiribati's economic well being, Tiimon instead argues that it is this very isolation that has allowed them to continue their way of life in which poverty does not seem to be a factor. In the same statement, Tiimon puts forward the argument that, "Kiribati is one of the poorest countries on earth, but because the people are so communal, there are no street peoples or beggars. Everybody shares and helps each other...The people have traditionally lived in a subsistence economy based 
on crops like taro, breadfruits, coconut and pandanus and fish for their protein. Sadly drought, storm surges, seawater inundation and coral bleaching have already had an impact on these traditional food sources” (Tiimon 2010: 25). Here, Tiimon presents a clear statement of responsibility and causality in which it is climate change that is jeopardising this way of life. The necessity of the international community taking responsibility for action on climate change is a clear message from both Alofa and Tiimon.

Alofa and Tiimon comment on Kiribati's economic ability to respond and it is apparent that issues of finance are key concerns for them. However in the way that both Alofa and Tiimon present their arguments for demanding climate finance, there is a comprehensive argument to be made in which their reliance on finance is based on concepts of responsibility within the climate change debate as opposed to a concept of Kiribati being innately vulnerable and dependent. This contrasts with the way that others have presented Kiribati's economic situation, noting Kiribati's small size and limited economic growth as a way of highlighting continued dependency. I would argue that in their [re] framings of social vulnerability, Alofa's and Tiimon's language bears a clearer alliance with Hau'ofa's interpretation of the Pacific as selfsufficient, interconnected and by no means dependent.

\section{Alofa and Tiimon Reframe the Debate}

As has been shown, both Alofa and Tiimon have used the same adjectives as those used by those from outside of the Pacific to articulate the vulnerability of Kiribati. The way in which these terms have been applied, however, creates a contrasting view of Kiribati’s vulnerability. Alofa and Tiimon make consistent reference to Kiribati’s small size in relation to concepts of space restricted to land mass, which, it could be argued, demonstrates Hau'ofa's argument of internalisation. However, Hau'ofa presents oppositional framings of the Pacific, in which the Pacific is viewed as either innately small and vulnerable or large and self-sufficient. In the context of the climate change debate, as has been argued above, the truth lies somewhere in the middle and a more fluid understanding of the framing of the Pacific is required. Both Alofa and Tiimon have drawn on terms such as small and low-lying to indicate that Kiribati is vulnerable based on biophysical frameworks in their truest form.

Alofa and Tiimon are clearly drawing on the language that has provided Kiribati with so much attention to give further emphasis to their demands for action; their use 
of similar language is an example of Barnett and Campbell's assessment of appropriation. However, the way that both women frame this call for action makes it explicit that, for them, Kiribati is vulnerable because of climate change, as opposed to climate change highlighting or exacerbating a pre-existing vulnerability. By framing Kiribati in such a way, they are consistently raising the issue of responsibility with the international community. This is no more evident than in Alofa's and Tiimon's calls for climate finance.

While both Alofa and Tiimon are calling for climate finance, the way they are doing this makes it clear that, first, climate finance should be delivered by those responsible because I-Kiribati themselves did not create these problems, and secondly, that climate change is the factor that is making them economically vulnerable. Alofa, contributing to climate witness statements held at the Oxfam climate hearing in Copenhagen 2009, made it apparent that while climate finance is demanded, it is based upon the responsibility of industrialised countries as opposed to dependency. This is illustrated in this statement, "The witnesses called for...developed countries to provide financial compensation to help developing countries to adapt to already occurring climate change...” (International Institute for Sustainable Development 2009: 1). The framing of the request as a request for compensation rather than aid reinforced the view that climate change is caused by others and that the responsibility lies with others to deal with the consequences.

The use of terms such as compensation raises some interesting questions about the legal, political and economic consequences of such language. If funding is to be recognised as compensation by the international community, then this would imply a level of responsibility. It is possible that, because of this, the international community continues to frame adaptation funding as aid as opposed to compensation. The fact that Alofa and Tiimon are framing climate finance as compensation challenges such deferral of responsibility, underscoring that it is the developed world's responsibility to help places like Kiribati made vulnerable by climate change. This message of responsibility is echoed throughout both Alofa and Tiimon's conversations. Both these women make it expressly clear that Kiribati is not innately vulnerable; rather, that climate change has produced a set of circumstances that has hindered Kiribati's economic viability.

It is interesting to note here that while reducing emissions is something that Kiribati cannot possibly do, as it is one of the countries that produces the least 
amount of emissions, and therefore calling for emission cuts from other countries is vital, funding for adaptation is positioned in the same way. Both Alofa and Tiimon present their arguments in such a way that indicates that the responsibility for both emission cuts and climate compensation falls on the shoulders of the international community. The necessity of the international community taking responsibility for action on climate change is a clear message from both Alofa and Tiimon. Tiimon in an interview argues,

I strongly feel that I-Kiribati people have very little contribution to what is happening in the world, to the planet. So industrialised countries, I feel that they have more, they are the ones polluting the earth and on the other hand we tiny islands are the first ones to experience the consequences of that... (Tiimon in "Sinking Paradise” 0:54-1:22).

This sits in clear contradiction to the ways in which those from outside have framed the Pacific's vulnerability. While she makes use of the familiar adjectives, small and low-lying, Tiimon's comments make it apparent that it is man-made climate change which is the pressure point that is creating vulnerability, as opposed to presuming it is an inherent attribute of smallness. It is this with this position in mind that Tiimon is reframing the debate and arguing for the international community to take responsibility and act. Tiimon repeats this sentiment in another statement in which she highlights the responsibility of industrialised countries to act, "My message and the message from the people where I come from is for industrialised countries to see us as human beings as well and do something about climate change right now - not tomorrow or next year..." (Tiimon in "Sinking Paradise" 1:25-1:38).

As has been illustrated throughout previous chapters, the climate change debate is fraught with political power balances/imbalances. Therefore when the Pacific is being spoken about with consistent reference to smallness, isolation and dependency, it would appear that Hau'ofa's argument holds real relevance. This is particularly evident when examining the language that both Alofa and Tiimon are using. Although both Alofa and Tiimon have reframed the debate to focus on the cause of Kiribati's vulnerability, and in doing so highlight the perceived responsibility of the international community to act, there is still on occasion a tone of dependency in both Alofa's and Tiimon's messages. Hau'ofa would argue this is created and then 
perpetuated through the use of descriptive language such as small, which he sees as derogatory and trapping the Pacific into a state of mind of dependency.

\section{Articulations of Women by Alofa and Tiimon: Vulnerable or Agents of Change?}

Women's vulnerability to climate change has been a consistent theme throughout the climate change debate. While there is a general agreement that there are, and will, be gendered consequences to climate change, as has been shown there are contrasting views on how women's positions should be articulated, particularly in relation to vulnerability discourse. Chapter two provided an overview of these arguments in which there is an obvious clash between labelling women as innately vulnerable or as agents of change. Chapter four brought these arguments to bear on I-Kiribati women and raised some essential questions about how women are being framed. In examining how both Alofa and Tiimon choose to explicitly identify or not identify IKiribati women in their discourse, the following section allows for these questions to be further explored.

In examining both Alofa's and Tiimon's statements about climate change, it becomes apparent that while the gendered consequences of climate change may be of great concern for those outside of Kiribati, from these I-Kiribati women there is very little specific mention of I-Kiribati women's vulnerability or agency. Surveying a wide range of publicly available statements from 2009 to 2011, there is only a handful in which Alofa mentions women, while Tiimon appears not to mention women at all. Alofa, giving an interview at COP16 states, "We say that Kiribati is the most vulnerable country, but in the most vulnerable country you have the most vulnerable people and that is the mothers, the women and the children...” (Alofa in Pelenise Alofa, President... 02:05-02:18). Alofa's statement echoes similar arguments that have been presented by Gro Harlem Brundtland, in which social frameworks of vulnerability have been applied. These have shown that, first, poor people are more vulnerable to climate change, and secondly, that women, who are over represented in poverty statistics, are even more vulnerable to climate change. These sentiments establish a perception that women's vulnerability is a quality born out of their gendered roles within society which climate change is exacerbating. This bears similarities to the way in which Pacific Islands have been articulated as vulnerable based on innate qualities. These similarities in depicting both islands and women will be explored in more depth in the concluding chapter. 
Alofa's statement would make it appear that women's vulnerability is an unavoidable quality, with little analysis of how women's gendered roles within society impact on the outcomes of climate change. However, Alofa clarified that she is identifying women's vulnerability in relation to the social roles for which I-Kiribati women take responsibility. This is evidenced in a statement Alofa makes in regard to I-Kiribati women, presenting the point,

When you talk about climate change the most vulnerable people in my country are the women and the children... In Kiribati it's the women who have to look after the home and the family...with climate change it is the women who are left to find increasingly scarce fresh water, who need to look after the babies when they get sick from dirty water, who repair the houses after storms (Ireland 2010: 1).

This is an obvious articulation in which women's roles within society are placing them in a distinctly vulnerable position. This statement made by Alofa articulates women's roles within society and the way in which climate change exacerbates these vulnerabilities. While Alofa's statement highlights the roles that women play in Kiribati that establish them as more vulnerable to the impacts of climate change, there is no mention of I-Kiribati women's ability to adapt to these circumstances in creative and innovative ways. It provides credence to arguments made by such people as Anita Nayar (2011) who argue that the continued reference to women and vulnerability crowds out other forms of analysis of women in particular as agents of change. These articulations of I-Kiribati women, while important in the fact that they are drawing attention to the gendered implications of climate change, clearly limit the way in which women can and are being viewed. This is also a demonstration of the way in which language that has been used to talk about I-Kiribati is being adopted and reflected back by the very women that are being spoken about in this way.

As can be seen, where women are being spoken about, it is in relation to vulnerability frameworks, as opposed to frameworks of agency. The basis on which scholars such as Philippe Bouncour have argued for women's vulnerability to be acknowledged and assessed is based on the idea that by doing this, women will gain a stronger voice within climate change debates, therefore creating policies that take gender into consideration. Alofa and Tiimon are popular spokespeople for Kiribati on climate change issues and are consistently referred to, interviewed and commented on 
as the examples of the human faces of climate change. For example, when asked why she is at COP16, Alofa states "I am here with my NGOs Pacific Calling Partnership and also with Oxfam to help to bring the human face to the climate talks while the negotiations are going on...” (Alofa in 'Pelenise Alofa, President...01:25-01:14). Tiimon is also presented as a human face of climate change; when delivering her speech at Copenhagen, Tiimon implores, "I am here to ensure that the human face of climate change and the voice of my people are not forgotten. We need civil society and world leaders to act on this issue. If nothing is done what will become of us? What will become of our children's children?” (Pacific Calling Partnership 2009: 1). Despite both Alofa and Tiimon's roles as spokespeople for Kiribati on climate change issues, the fact that there is limited published material on the gendered implications of climate change by either woman appears to give credence to an argument made by Sandra Grey (2006) in which she questions any automatic assumption that women will speak for women.

It is also interesting to note that when Alofa does mention women, it is their vulnerability that is referred to. Alofa's articulation of women's vulnerability is used in the same way that Kiribati's vulnerability is being used, as a way to encourage action from the international community. However, Alofa does not appear to highlight women's vulnerability in order to argue for gender responsive policy solutions. This appears to diminish the arguments that have been used to defend the articulation of women as vulnerable as a way of increasing women's voices in the climate change debate and resulting in greater awareness of the need for gender sensitive policies. The limited attention that Alofa has paid to I-Kiribati women's position, and the apparent lack of specific acknowledgement that Tiimon has placed on women's position in regard to climate change, shows that increases of women's visibility does not necessarily make for a direct link to increases in gender sensitive policies or attention.

The fact that both of these women are depicted as the face of climate change, while drawing very little attention to I-Kiribati women's positions in relation to climate change, raises several interesting questions. Can it be argued that, by presenting themselves to the international stage as the human faces of climate change, that women's vulnerability should be automatically assumed? Or can it be argued that, as popular spokespeople for Kiribati at both the local and international level, mean that as agents of change, through their spokesperson roles, the fact that they 
rarely mention women is of no consequence? Or are they (as high profile women advocates) agents of change regardless of whether they specifically draw attention to the situation of women?

\section{Theoretical Explanations of Alofa's and Tiimon's Apparent Limited Attention to I-Kiribati Women}

Although it is important to consider these questions and acknowledge the lack of attention that Alofa and Tiimon have brought to I-Kiribati women specifically in regard to climate change, it is also important to understand possible explanations for this approach. The Third Global Conference of Women, Politics and Governance noted that, "The climate change debate is an indicator of how gender issues tend to be omitted, leaving room for complex market driven notions equated in terms of emission reductions, fundability and flexible mechanisms” (Third Global Congress of Women Politics and Governance 2008: 2). As has been shown, Alofa and Tiimon have focused a large amount of attention on the politics of climate change and argued for action based on this. Alofa, for example, has continuously called for emissions reductions and for funding to be made available for Kiribati. For both women there has also been a consistent aligning of arguments between themselves and the international community, in both the language they use and the attention they pay to particular issues, such as mitigation or adaptation arguments. A report by ISIS notes, "To date gender issues have hardly figured in the international policy discourse on climate change and have been often overlooked in discussions about strategies to reduce the source of greenhouse gases because of the 'technical' or 'scientific' nature of the strategies" (ISIS 2012: 1). Something to consider then is that a positioning of women's issues at the forefront of the climate change debate may be presumed to detract from the attention and support the debate has received. This reflects a sort of hierarchy of urgency where specific attention to women's issues falls down the list in the face of the sense of immediate and pressing danger of the broader issues for climate change as a whole. The same reduction of priority seems to apply to economic development initiatives focused on women. Arlene Griffen, facilitator of a 2005 workshop entitled "Defining Pacific Feminism," sums some of the discussions that were generated at this forum, "It was noted that women's projects were often not provided with resources by governments because they were thought to contribute little of economic value to the nation” (Griffen, A 1989: 108). 
Just as climate change knowledge has been largely generated from outside of the Pacific, a large amount of the research on gendered impacts of climate change, the framing of women within these debates, and the critiques of the lack of women's issues being vocalised, has been done by women and feminists outside of the Pacific. It is therefore important to consider Alofa's and Tiimon's limited acknowledgment of the gendered implications of climate change from Pacific and indigenous feminist standpoints. One possible explanation is that, for Alofa and Tiimon, ethnicity and nationality are more relevant here than gender. As noted in Chapter three, there has been a certain amount of criticism levelled at white and western feminists for making assumptions about inequalities of gender that all women face. For example, in her discussion of the US, Andrea Nightingale notes that "Latino and African American women argued that in many contexts race was more salient in shaping their experiences of inequality...” (Nightingale 2006: 6). These arguments have claimed that in many instances people of colour have had to fight battles in which there is a clearer affinity along ethnicity or nationality lines rather than along gendered lines, and that western feminism has often overlooked the impact that race has. Within the climate change context, this is a particularly relevant point to note when issues of power and politics are crucial factors and are being viewed through geographical locations.

Alofa and Tiimon have used the argument that somebody else has created the position that Kiribati is in. This may lead to framing the issue as one that is caused by one group (the west) imposing hardship on another (the Pacific). This in turn may lead to reluctance to separately identify women as an affected group. The framing of the problems that Kiribati and the Kiribati people are facing due to climate change would indicate that both Alofa and Tiimon are viewing this problem as one in which it is more important that all I-Kiribati are treated as one group.

Such decisions to frame indigenous issues in nationalist, rather than explicitly gendered, terms, are also evident elsewhere in the Pacific. For example Haunani-Kay Trask, a Native Hawaiian sovereignty activist, academic and intellectual, argues, "Pacific Island women, like Hawaiian women, seek a collective self-determination. That is to say, we want to achieve sovereignty through and with our own people, not separated from them as individuals or as splintered groups. Such individualism and separation promise only more confusion and more alienation, the very maladies which so afflict industrial peoples” (Trask 1993: 263). Although Trask is not 
specifically talking about the climate change debate, it becomes apparent when examining both Alofa's and Tiimon's statements that, for them, Kiribati is in a battle for cultural survival based on arguments of human rights. This is evident in a presentation from Tiimon where she questions, "How long will these cultural traditions last if we are forced to move away from our homelands as a result of climate change?” (Tiimon 2011: 17). When viewed in light of Trask's arguments, the framing of the argument in this way can account for the lack of attention to the specific circumstances faced by I-Kiribati women. Alofa has obviously acknowledged the role that women play within Kiribati society and how this creates vulnerability, but within the context of climate change it may be her view that it is more important to fight this particular battle as I-Kiribati people as opposed to IKiribati women.

\section{Conclusion}

This chapter has illustrated that the articulations of both Kiribati and I-Kiribati women presented by Alofa and Tiimon are not bound by the absolute articulations of vulnerability or strength presented in earlier chapters. In articulating Kiribati's position within the climate change debate, Alofa and Tiimon are drawing on vulnerability discourses in order to draw attention to their plight, yet they are reframing this vulnerability on their own terms. Alofa and Tiimon have consistently challenged the concept of Kiribati being innately vulnerable, instead highlighting how climate change is making them vulnerable. Their reframing of Kiribati's vulnerability in this way allows for climate change-related policies to be thought of in different ways. This can be seen in the way that Alofa and Tiimon have raised concepts of responsibility in relation to the international community's responses to Kiribati's situation.

Although considerable attention has been paid to women's vulnerability to climate change, Alofa and Tiimon have seemingly opted to articulate I-Kiribati plight as a national issue rather than highlighting gendered issues. While Alofa does, in the few instances where she talks about women, refer to their vulnerability, it appears this is done to further her argument about forcing the international community to take responsibility and act as opposed to highlighting specific gender inequalities. Finally, Alofa and Tiimon are powerful agents of change, in their role as popular spokespeople both within their communities and outside their communities, whether 
they are talking about I-Kiribati women's specific issues does nothing to diminish this role. It is through examining the way that both Alofa and Tiimon articulate Kiribati and I-Kiribati women's situation that it becomes apparent that concepts of vulnerability that bind Kiribati into one or another framework are unhelpful and that in fact a more fluid understanding of vulnerability and strength is required.

Chapter four presented the argument that it is precisely this type of language that has led to the view that forced migration is an inevitable outcome for Kiribati. This will be further explored when we look specifically at references to forced migration made by Alofa and Tiimon. Despite both Alofa and Tiimon arguing that forced migration should not, and does not, need to be the inevitable conclusion, the reiteration of the very language that outsiders have used to create a sense of the inevitability of forced migration is being repeated by Alofa and Tiimon. 


\section{Chapter Six: Articulations of Forced Migration by Pelenise Alofa and Maria}

Tiimon

Forced migration from places like Kiribati as a result of climate change has become an image that has proliferated throughout the climate change debate. The reality of continued changes to the climate has led to an almost uncontested expectation that, for low-lying islands, the ultimate outcome will be islands that are completely inundated by rising seawater. Scientists, international organisations and governments, including the Kiribati government, have used these predictions as a way of highlighting what inaction will lead to.

While it is true that sea levels are rising and that this is having dramatic and detrimental effects on Kiribati, I have argued that the way in which Kiribati is presented and described within the climate change debate has created a scenario in which forced migration is presented as an inevitable conclusion. This is due to understandings of Kiribati's innate vulnerability—understandings informed by more than just the science of climate change. The continued use of language that presents Kiribati as small, isolated, etc, reinforces long-held views of the Pacific as dependent and establishes forced migration as a likely result of this. Chapter four argued that it is articulations of places such as Kiribati in this way, coupled with the science, that has led to forced migration becoming the main focus of discussions, and in some cases to it being presented as a positive option at the expense of other forms of adaptation and a real commitment to mitigation by the international community. Forced migration may indeed become a preferred option as the international community considers the cost of mitigation and adaptation. The pragmatic rationales of major industrialised nations-hesitant to cut their emissions-may echo the thinking underlying the infamous comment by then US Secretary of State Henry Kissinger regarding the atomic testing in the Marshall Islands: "There are only 90,000 people out there. Who gives a damn?” (Vine 2009: 183). Thus, conceptions of smallness and remoteness become justifications for momentous policy decisions that, in turn, lead to scenarios of forced migration.

However, as has been shown in Chapter five, Pelenise Alofa and Maria Tiimon have presented Kiribati in a different light. Although drawing on some of the same 
language there is a contrasting message being delivered; while emphasising that Kiribati is vulnerable to climate change, both women clearly link this vulnerability to the actions (or inactions) of the international community, as opposed to the innate vulnerability of their island nation Kiribati. And rather than seeing Kiribati's future as one to be determined solely by economic imperatives, they refer to the human right to survival. While both Alofa and Tiimon present Kiribati in a different way, it is also clear that forced migration is a scenario that both are taking seriously and both invoke it often to demonstrate the risk that they face. This chapter seeks to explore how Alofa and Tiimon are articulating ideas about forced migration, and examine if they are presenting the same level of inevitability. In order to do this I will look at how Alofa and Tiimon present the problem of forced migration, and in particular the causes, and the degree to which the inevitability of forced migration features in their discourse. Lastly, this chapter will consider what Alofa's and Tiimon's articulations of forced migration means for I-Kiribati people.

\section{Causes of Forced Migration Articulated by Alofa and Tiimon}

International organisations are using images of 'sinking islands' forcing I-Kiribati to flee for their lives as a way of demonstrating the human cost of inaction. While it can be argued that these concepts and images have generated a vast amount of attention for places such as Kiribati, there is a risk that the true cause of what will force people from their homes is overlooked. At the same time, these images create an idea that this is something that will happen in the future as opposed to looking at what is happening right now. It could be argued that this reliance on presenting a future scenario of forced migration serves the interests of the international community as a way of delaying action. This was evidenced in Chapter four where it was shown that the international agreements around mitigation strategies are based on 4 degree warming, despite calls by Small Island States to reach a binding 1.5 degree commitment which, it is argued, would prevent a considerable amount of the damage. As has been shown in Chapter five, both Alofa and Tiimon have reiterated this call in their various presentations and speeches.

Alofa and Tiimon have been questioned about and invoked forced migration as an inevitable outcome for Kiribati. However, the way that both these women talk about forced migration implies that it is not complete inundation some time in the future that Kiribati is most vulnerable to; rather, it is in fact the already occurring 
salinisation of fresh water and soil that is creating a situation whereby island living is becoming untenable. This can be seen in a speech made by Alofa in 2009 in which she urges New Zealanders to act on the behalf of Kiribati. She summarises the impacts of climate change and its results, arguing,

With climate change they're having problems with their water and most of their coconut trees are dying. They have no leaves and no fruits that is caused by the very high temperatures or drought, there's no rain and the salinisation of soil. Seawater has come in...You cannot grow vegetables you can grow nothing. The water supplies in the villages is well water. When sea water seeps or comes into the well water it becomes salty and it's not drinkable, it's unhealthy for our people... (Alofa in '100 days to Copenhagen.’ 0:23-1:02).

This presentation of what is happening to Kiribati by Alofa is used to demonstrate that the impacts of climate change are not a problem for the future but are in fact a problem today that I-Kiribati are already having to deal with. The illustration of climate change impacts in this way challenges both the belief that radical mitigation and adaptation policies can wait and that it is only total inundation that will force people from their homes.

This is a strategy that Alofa employs on several occasions and can be seen again in an interview that she gives with Phillip Ireland in Cancun in 2010, where she reiterates,

People are already being moved and resettled...Just recently some friends of mine were forced to leave their islands because all the sea water comes over the land and poisons our food crops and fresh water holes with salt...We have not seen these things before. It is hard for our people to cope (Ireland, 2010: 1).

Alofa makes it clear that forced migration is already occurring for her people, and, while it may not be the headline-grabbing migration created by total inundation of rising sea levels causing islands to 'sink,' other effects of climate change are nonethe-less causing people to relocate as their ability to continue to have a viable economic future on the islands is being eroded. Statistics produced by the Kiribati National Statistics Office (2005), cited in Chapter four, support both Alofa and Tiimon's claims that Kiribati has experienced an increase in migration from outer 
islands to South Tarawa. Demonstrating what is happening right now to Kiribati, caused by climate change, allows for activists such as Alofa to inject a level of urgency into calls for action, while some in the international community are still debating the reality of climate change or what impacts it may have. By highlighting what is already happening in her country Alofa is able to give her calls for action more credence and immediacy.

Chapter four showed that there remains some argument over the causes of migration. To reiterate, scholars such as Camillo Boano, Roger Zetter and Tim Morris, writing for the Refugee Studies Centre, point out, "Migration, and population movement in general, is part of human history and an important adaptive mechanism. Thus, it has always been difficult to differentiate 'environmental refugees' from ‘economic migrants'” (Boano, Zetter \& Morris, 2008: 5). While there may be some distinction in academic circles between environmental and economic migration, it is clear from the above statements by Alofa, and similar statements from Tiimon, that there is little distinction between economic and environmental migration in their minds. Tiimon makes this abundantly clear in a speech made in 2010:

Of course we only wish that our submergence will never be a reality. But even if they are not submerged, their ability to sustain life will be diminished. As fish is central to many economies and diets, people of Kiribati and low lying islands will be the most affected as they have a very limited ability to develop other sources of income and food in the face of such change. (Tiimon 2010: 1).

In accord with Alofa's arguments, Tiimon's statement makes it clear that while total submergence of her island nation Kiribati may be something that I-Kiribati have to face in the future, the real problem is the ability to sustain life through gradual sea level rise.

Tiimon's statement also makes it apparent that, for I-Kiribati, environmental and economic reasons for migration are intertwined. Climate change is generating a situation that is making it economically not viable to remain living on their islands. This is a sentiment that Tiimon repeats in 2011 in an article entitled, 'A human face of climate change.' Tiimon asserts,

The people have traditionally lived in a subsistence economy based on crops like taro, breadfruits, coconut and pandanus and fish for 
their protein. Sadly drought, storm surges, seawater inundation and coral bleaching have already impacted on these traditional food sources. Several communities in Kiribati have had to move and more can see that they will need to move in the future. (Tiimon 2011: 1).

The statements made by both Alofa and Tiimon make it clear that their primary concern is not their islands sinking under increasing sea levels some time in the future, but rather that climate change is at present causing sea levels to rise, creating a situation where continued living is becoming increasingly untenable for I-Kiribati. The way that these images are presented illustrates that both women are using the reality of life on their islands as a way of challenging the international community to act now rather than some time in the future, as the impacts of climate change are real and taking effect now. Alofa and Tiimon also underscore that climate change is making it economically harder to remain living on their islands and that this is causing people to move. However, unlike some scholars, for Alofa and Tiimon the distinction between environmental and economic causes of migration appears to be blurred; the two causes are so interconnected that there is little difference. It is interesting to note that while in earlier discussions both Alofa and Tiimon have used discourses of danger to articulate Kiribati's situation when talking about migration, they are consistently staying away from images of sinking islands and instead presenting the facts about what Kiribati faces, which both argue is just as damaging as their islands becoming submerged by future increases to sea water levels.

\section{Inevitability}

Both Alofa and Tiimon have also placed the causes of migration within a wider political context in which inaction of the international community is expressed as a real cause for the possible forced migration of I-Kiribati. Alofa draws on similarities between possible forced migration and the $20^{\text {th }}$ century relocation of people from the island of Banaba in Kiribati as a result of phosphate mining. The British Phosphate Commission, led by a board of British, Australian, and New Zealand representatives, mined Banaba so extensively that it forced the people of Banaba to be relocated to an island off Fiji. Alofa argues that the causes for that migration and the possibility of IKiribati being moved again can be attributed to the same motivations. Alofa argues, "So why did they move them [Banabans]? Put it in simple words, it was economics 
and money and today if my people are going to be displaced for a third time it is [for] the same reasons, and that is not acceptable and it is greed" (Alofa in 'Voices of the Vulnerable' 2:58-3:16). This statement indicates that while the international community sees climate-induced sea level rise to be the main factor causing the possible migration of people, Alofa is framing relocation within the wider economic and political context in which climate change policies are being debated. For Alofa there is a clear connection between what happened to Banaba and Banabans and what is happening again with regard to climate change. For her, invoking the Banaban situation is a powerful rhetorical device for delivering the messages that she wants to express, as can be seen in this statement made by Alofa,

Today, I relate CC [climate change] negotiations to the experience of my own people the Banabans. If nothing is done and the people get resettled, it is because CC negotiations is not about humanity, but economic development (money). The pursuance for development by most developed countries is not about sustainability for everyone, but the conspiracy by the few rich people (Alofa. 2011: 1).

In this statement, Alofa makes a link between the relocation of Banabans and the possible relocation of I-Kiribati today. Alofa uses this example to illustrate the connection between the economic development of others and the forced migration of I-Kiribati today, with an obvious signalling that, for her, the inevitability of migration is based on considerably more than the innate vulnerability of Kiribati.

Tiimon also reiterates this framing of causes of migration. Tiimon does this by using a quote from President Tong: "Some industrialised countries might be arguing on climate change would hurt their economic development. Sadly I say NO. It is not an issue of economic growth it is an issue of human survival” (Tiimon 2010: 1). Both Alofa's and Tiimon's statements show that, for them, climate change is not happening within an isolated understanding of the environmental impacts. Rather, they are addressing the political and economic forces that are shaping their futures. This construction of migration does not assume it to be a natural outcome of Kiribati's innate vulnerability. Rather, the possibility of forced migration is an outcome that is based on the created vulnerability of Kiribati as an island state with limited political power within the climate change debate; in other words man-made climate change produces man-made vulnerability. These alternative depictions of 
causality have created a different construction of the inevitability of forced migration from Kiribati. Although both Alofa and Tiimon are acknowledging that migration is an outcome which I-Kiribati are facing, and total forced migration may be something that they face in the future, their articulations of what the causes are creates a different image of the inevitability of forced migration.

As was argued in Chapter four, concepts and portrayals of Kiribati's vulnerability have led to forced migration being presented as an inevitable outcome for Kiribati. However when we look at the messages that Alofa and Tiimon are presenting at international forums, it is clear that there is a level of hope-hope that the international community will hear their voices and act before Kiribati has to consider the option of forced migration. Alofa's and Tiimon's statements emphasise that forced migration does not need to be an inevitable outcome provided that there is strong commitment by the international community to lower emissions. This can be seen in a speech by Alofa, in which she says,

Because I have to accept the fact that the water is coming up, I have to accept that, but just because water is coming up doesn't mean I have to turn and run, problem comes up and all of a sudden all of us have to turn and run, NO, we have to come up with a solution...ok water is coming up, ok solution one cut the emissions, two provide the technology provide the money and that's it... (Alofa in 'Climate Testimonies. 0:29-1:01).

Alofa makes it clear that, while there is an acknowledgment that with continued sea level rises the risk to staying with their land increases, forced migration does not need to be the ultimate outcome for them provided that the international community acts.

Chapter four presented the argument that there is an increasing consensus of views in the science of climate change, and that any increase in warming above 1.5 degrees will spell almost imminent devastation for Kiribati. This is a message the Pacific community has consistently delivered to the world stage. As has been shown in Chapter five, both Alofa and Tiimon have made consistent pleas to the international community to cut their emissions. These calls are based on the available science, which suggests that without these cuts, the total forced migration of populations from places such as Kiribati will undoubtedly become a reality. Alofa's and Tiimon's speeches since 2009 underscore that, despite limited action thus far on reaching a binding agreement, the call for emissions cuts is still a message that they 
are delivering, with the belief that this action will eliminate the need to leave their islands. Alofa at the Cancun climate talks in 2010 states,

My message, my plea, I'm asking the negotiators here in this country, who are all here and Australians who are back in my country helping...We do not just need money, we need Australia to say we will move from fossil energy to green energy, we will invest our money into green energy, we will cut our emissions so that the most vulnerable countries like Kiribati and Tuvalu will continue to live in their homes and their culture and speak their languages and to look after their children back in their homes. So that is my message. (Alofa in 'Pelenise Alofa talks about the human... 3:14-4:01).

This passage highlights that, for Alofa, forced migration of her people from her country is not an inevitable outcome of climate change provided that the international community takes steps to lower their emissions. This call for emissions reduction is based on an understanding of the science in which anything less than what the Pacific commentators are asking for will have disastrous effects on their countries.

Alofa repeats such calls on several occasions, linking her demands for mitigation with the current science. Alofa also demands that her own government continues to call for the international community to take action to mitigate the effects of climate change. In 2010 Kiribati hosted the Tarawa Climate Change Conference for Pacific leaders and invited those from the wider Pacific region such as New Zealand, Australia and China, to attend. Alofa outlined the calls that she and her organisations would be making at this event, which remains a call for her government to continue to negotiate for emissions cuts. Alofa states, "We will urge the government to continue to stand strong and call for an international climate treaty that will limit warming to $1.5 \mathrm{c}$, and sets a target concentration of carbon emissions to 350 parts per million, because that is what is safe for our people” (Alofa 2010: 1).

Tiimon is also echoing Alofa's calls for emissions cuts based on the science. Tiimon urges,

I also beg you to urge your governments to work towards achieving a global commitment to reduce greenhouse gas levels to 350 parts per million by 2020. Finally, let us all work towards legally binding agreement that effectively reduces greenhouse gas 
emissions and ensures that no party is left behind. (Tiimon 2010:

1).

Both Alofa and Tiimon, by continuing to argue for legally binding agreements that will bring emissions to a point that will allow for I-Kiribati to stay on their islands, indicate that despite the limited action that has so far been agreed upon by the international community, they are refusing to view forced migration as an inevitable outcome.

Where forced migration is being talked about as inevitable, both Alofa and Tiimon have re-framed the debate to argue that forced migration, if it does eventuate, will result from inaction by the international community as opposed to some inherent destiny for Kiribati. As Alofa puts simply, "Some people say it is too late, I feel well it's not, we're just not doing anything, and so it's going to be late see, we're not doing anything” (Alofa in 'Pelenise Alofa, President...'12:07-12:17). Alofa is here expressing frustration with that fatalistic acceptance and lack of action despite the widespread acceptance of the scientific evidence of the causes of climate change. This frustration can also be seen in statements Tiimon has made in which she implores, "Eventually the majority of us I-Kiribati will have to leave our islands. Climate change must be addressed from a view point of human rights” (Tiimon 2010:1). In statements such as these Tiimon indicates that while there are continued calls for action, there is limited faith in the process. Such statements would appear to indicate recognition of the inevitability of forced migration. However, I would argue that statements such as these-while indicating a level of resignation about what is achievable-are in fact not intended to indicate a fatalistic acceptance of the inevitability of forced mass migration, but rather to highlight what inaction will result in for Kiribati and I-Kiribati.

The acknowledgment that the international community may not act in time has led to discussions of how best to manage the migration of I-Kiribati people. While both Alofa and Tiimon have continued to push for emission cuts, both women have expressed fear over what migration will mean for their culture. The expression of this fear has apparently influenced the conversations that they are having about how best to migrate if some or all I-Kiribati have to migrate. 


\section{Impact of Forced Migration}

Although the international community has positioned forced migration as an almost inevitable outcome for places such as Kiribati, conversations are also being generated around the potential impact that this mass forced migration will have on I-Kiribati people. Many have researched and commented on the classification of what counts as environmental forced migration, the legal consequences of forced migration due to environmental degradation, the economics of such migration, and security risks that this type of mass migration might create. Despite both Alofa's and Tiimon's continued calls for emissions reductions, it is apparent that at some level the reality of forced migration has a bearing on their conversations. While the environmental, legal, economic, and security considerations are important to take into account, it becomes clear in the dialogue of both Alofa and Tiimon that, for them, the most pressing focus is on the question of what will happen to I-Kiribati culture, history, and identity as a result of physical disconnection from their land.

This is an important point to highlight when talking about forced migration. As has already been noted, there is a long history of migration within and outside of the Pacific. Drawing upon this history of migration, some argue that migration, as an adaptation strategy, does not need to be viewed in such a negative light. As was shown in Chapter three, scholars such as Hau'ofa have presented compelling images of a Pacific in which historical migration has always been used by Pacific Islanders as a way of forging connections and creating economic links. Hau'ofa situates current processes of migration within a great tradition of movement within and across the Pacific, pointing out that Pacific peoples "have since moved, by the tens of thousands, doing what their ancestors did in earlier times: enlarging their world, as they go, on a scale not possible before” (Hau'ofa. 2008: 34).

Hau'ofa's argument that migration has a constructive place within Pacific history has been used by academics such as Barnett and Campbell to present a counter view of migration within the Pacific. Barnett and Campbell argue, "Much of the migration in the world is voluntary and leads to positive outcomes for migrants, the places they come from and the places they move to, suggesting in turn that in some instances migration may contribute to adaptation” (Barnett \& Campbell. 2010: 171). Barnett and Campbell are broadening the discussion of migration related to climate change in an attempt to highlight possible positive outcomes of climate-induced migration. Barnett and Campbell reference historical migration patterns that are evident within 
the Pacific, and particularly highlight the benefits of remittances, for example, to elaborate the benefits that can come from migration.

Pacific history is made up of migration both within the Pacific region and broader afield, and the impact on culture and identity has been significant. In light of this migration, cultural identity becomes a fluid notion, continually changing, merging and adapting. As Tammy Tabe sums in her thesis work on the I-Kiribati community resident in the Solomon Islands, "For centuries, the constant mobility and migration of individuals and groups of people have continuously shaped people's identities. This construction and reconstruction of identities is one of the most significant elements of diasporic communities, whether they resettle voluntarily or involuntarily” (Tabe 2011: 75). Tabe's work highlights how senses of culture can persist, even in radically different new environments.

Although these arguments can be viewed as an attempt to re-frame discussions of migration, they are made with reference to contexts in which there is a continued connection to a homeland. This is in contrast to a possible Kiribati scenario, where the "homeland" physically ceases to exist in any habitable way due to sea level rise. This raises questions regarding how I-Kiribati can continue to have a strong cultural, historical, identification with a homeland that is no longer physically there. Both Alofa and Tiimon have spoken about forced migration in a context in which the loss of their land is equated with the loss of their culture and their identity ${ }^{2}$. At the 2010 Tarawa climate change conference held in Kiribati, Alofa writes,

My message for the delegates at the Tarawa climate change conference (TCCC) is that they must give us hope. They must give I-Kiribati-my countrymen and women-the hope that we can continue to live in our islands. They are negotiating on an issue that is a matter of life and death. When I say death, our people will not die, but our culture and way of life will die and that scares me. (Alofa. 2010: 1).

Alofa's statement indicates that for her there is a real fear that if I-Kiribati are forced from their homes then their culture will be lost. Framing the cost of forced migration in this way highlights the importance that Pacific Islanders attach to their land. While

\footnotetext{
${ }^{2}$ For a related discussion of Banaban concepts regarding interconnectedness of land, bodies, and cultural identity see Katerina Teaiwa (2005). Additionally, Mike Roman is currently undertaking PhD studies at the University of Waikato looking at the impact of migration on I-Kiribati language and culture - when published this work will provide further reading on this point.
} 
the international community may be framing the climate change debate using images of islands sinking to indicate the gravity of the situation, Alofa appears to be taking this one step further and talking about more than just loss of land but also loss of IKiribati identity and culture. Alofa reiterates this point when speaking at the Pasifika festival in Auckland in 2011, articulating the impact of the loss of Pacific islands in this way,

Every Pacific Islander that I meet and I greet and I say where do you come from, they say I AM Kiribati New Zealander, I AM Samoan New Zealander, I AM Tongan New Zealander, why do you put the name of your country first before New Zealand? Because that is your identity. When you return home they say this is the sons and daughters returning home, this is the son of Kiribati, this is the son of Samoa, how can you be a son of Samoa, how can you be a son of Fiji when there is no more Samoa and there is no Fiji, there is no Tokelau, there is no Kiribati, there is no Tuvalu. (Alofa in 'Pelenise Alofa @ Pasifika...0:01-0:42)

By articulating the impact of migration caused by climate change in this way, Alofa is making a clear connection between Pacific Islanders' identity and perceived relationship to their land, and emphasising that the loss of this land entails a loss of their culture. It is also interesting to note that within this same statement, Alofa is indicating that while migration happens already, there remains a close connection to people's homelands, which enables a dual identification as both, in this instance, an IKiribati and a New Zealander, or a "Kiribati New Zealander." Tammy Tabe, in her thesis looking at the I-Kiribati community in the Solomon Islands, in which she interviews I-Kiribati who were relocated there during the 1950s and 1960s, notes how this resettlement has impacted upon their cultural identity. While Tabe identifies how I-Kiribati identity is a more fluid notion for those who have been resettled, Tabe notes,

Land is highly valued and is of great significance in Kiribati society. It is indicative of wealth, prestige, and security. The settlers' relocations uprooted them from the land on which they had established themselves, land that they are linked to by culture, economic, social, and traditional ties. Land is an essential resource for societies and it is vital for settlement, subsistence and 
economic development. It exists as a source of identity, power, authority, and status and has physical, spiritual, and cultural values (Tabe 2011: 57).

While acknowledging that culture always shifts and changes according to environment and circumstances, so some kind of I-Kiribati culture would certainly persist and exist outside of Kiribati (as Tabe’s work demonstrates) what Alofa signals here is her strong desire to retain the option of the type of I-Kiribati culture that is inextricably tied to the Kiribati land base.

This is a point that Katerina Teaiwa has made in her article 'Our Sea of Phosphate: The Diaspora of Ocean Island’ arguing,

...Human identification and activity across the region [Pacific] was marked by both the rooted and the stable, as well as the travelling and dynamic. This apparent binary was not oppositional or paradoxal, but simultaneously at work in shaping identity and fuelling and sustaining social life. Pacific identities were maps connecting places and often traversing oceanic space...Identity maps are thus created that are firmly rooted in multiple and specific places (Teaiwa 2005: 173).

While Teaiwa notes the way in which Pacific identities have been shaped by great migration, within the context of this article, she is arguing that for Pacific Islanders, identity starts from the land and that through the exploitation of this land there has been an exploitation of the people of the Banaban Islands, causing a loss of their culture which is connected to the land. While it is true that I-Kiribati living cultures are dynamic and adaptable, in Alofa's speeches she is making reference to the specific cultural ties that I-Kiribati have to Kiribati land and questions the impact that losing these lands will have on I-Kiribati cultural identity.

Alofa has drawn attention to the impact that the loss of land will have on Kiribati culture. She has done this in fora that are directly speaking to Pacific communities. Tiimon has also identified what it means to I-Kiribati to be talking about losing their homes with similar reference to the loss of culture that forced migration would result in. She has taken this message to the international stage. Speaking at a Kiribati side event at the Cancun climate talks in 2010-where a Kiribati dance was performed to open the talks-Tiimon declares, "We are not just entertaining you today but what we are trying to tell you today is, these are the cultures that we are going to lose if we are going to disappear because of climate change, these are one of the cultures, our 
dancers" (Tiimon in 'Pacific Calling Partnership...0:33-0:49). The way in which Tiimon uses the loss of her culture is similar to that of Alofa in that she is identifying a far greater risk to I-Kiribati people than just losing their land. This can be seen again in a Tiimon speech when she argues,

We love and treasure our culture, our traditional way of living, and our special skills that our ancestors have been passing them from generation to generation. These are part of us, they are our identity. Losing our identity is that last thing on earth we would want to happen. So climate change is not just about relocation, or migrating people to a safer place. It's about human rights, justice and human survival" (Tiimon in 'Pacific Calling Partnership...0:33-0:49).

Tiimon again highlights the connection between I-Kiribati and their land. This statement can also be seen to be using the loss of culture as a way of bringing a human face to climate change. While the international community may be negotiating on how much or little they should cut emissions by, and the economic impacts of such decisions, Alofa and Tiimon make the point that, for I-Kiribati people, the loss of their land means the loss of their history, culture and identity. This keeps the human cost of the results of climate change negotiations centred in their discourse.

As has been shown, the way in which both Alofa and Tiimon have articulated the cost of forced migration displays a marked difference to the way in which the international community is articulating the cost of forced migration. The following section demonstrates how this concern regarding loss of culture and identity has influenced the way that Alofa and Tiimon have framed suggestions for how to best migrate people if that is the final option.

\section{How to Migrate if Forced to Migrate}

Although Alofa and Tiimon continue to call for emission cuts in order to prevent the inevitable total migration of I-Kiribati people, they have also indicated a level of resignation to the fact that this may be the only option left for them. However, their discussions of the risks this poses have been articulated in considerably different ways than the international community has framed the forced migration discussions. In contrast to an economic argument, Alofa and Tiimon have brought attention to the fact that when discussions about forced migration take place, they should not just be 
about people having to leave their homes, but also about people having to leave their traditions, cultures and identities, all of which are formed and continued through their connections to their land.

The concern that both Alofa and Tiimon have demonstrated over the impacts of the possibility of forced migration has led to them delivering messages about how best to manage migration, if that is what the final outcome is, in a way that keeps their culture intact. Alofa, in an interview with Simon Butler from Greenleft in Australia, was asked "What do you want the Copenhagen conference to achieve?" She answered,

...A proper plan for those who are relocated. If people have to move somewhere [because of climate change], they cannot just move people, no. We have to have to think of their culture, think of their identity, think of their language, think of everything that makes them a people, a community. They have to have a proper plan (Butler. 2009: 1).

Alofa's statement makes it clear that while migration is not a desired outcome, it may become the only option left for I-Kiribati people and that in order to preserve Kiribati culture there needs to be planning in order to create the best scenario in which this happens.

Planning is obviously a pivotal point for both Alofa and Tiimon, as Tiimon has also commented on this. For example Tiimon argues, "In the future as our islands become uninhabitable, the people of Kiribati will need to move into other bigger countries, but we want to move with dignity and respect, as skilled migrants, contributing to the country. So we need to start now with education that provides skills to everybody not just the lucky few” (Tiimon 2010: 1). Tiimon is advocating for planning in much the same way as Alofa; however they have markedly different perspectives on what should happen. In Tiimon's statement, she is arguing for early migration to take place through education and training in host countries. This proposition-to have migrant communities established through skilled migration into places such as Australia and New Zealand-clearly echoes the calls that President Tong has made about this issue. Tong has repeatedly talked about 'migration with dignity’ as a way of achieving an acceptable form of migration. For example Tong states, 
The science is clear-climate change threatens the long-term survival of Kiribati. As such it would be irresponsible to acknowledge this reality and not do anything to prepare our community for eventual migration in circumstances that permit them to migrate with dignity...The concept of 'migration with dignity' is crucial to the effectiveness of the government's relocation policy. I-Kiribati migrants should be sought after by the countries to which they wish to relocate. For this to happen our people must be in a position to provide the skills that are needed in the receiving countries. This creates a win-win situation, where both Kiribati and the receiving country benefit (Tong 2012: 1).

This policy of relocation with dignity is intended to allow for pockets of I-Kiribati communities to be established. Tong expands on and qualifies this by stating, "This will assist in establishing expatriate communities of I-Kiribati, who will be able to absorb and support greater numbers of migrants in the longer term” (Tong 2012: 1). The concept of establishing outer-national Kiribati communities, in order to better make the transition from Kiribati to other host countries, can be seen to help keep cultural and traditional ties intact and to assist in the integration of subsequent waves of lesser-skilled and economically inactive migrants.

While Tong has said that relocation is a last resort, statements such as these indicate that, certainly for him and the Kiribati government, forced migration is an outcome that they view as inevitable and one that requires a plan of action. Tiimon, by repeating Tong's calls for migration with dignity, could also be seen as creating a sense of inevitability. However, does this reiteration of 'migration with dignity' automatically indicate vulnerability? The framing of migration through a focus on skilled migrants able to offer skills to the country of their choosing indicates that there will be a level of choice in the migration process. The use of the words such as 'respect' and 'dignity' indicate that through this type of migration, these attributes will remain intact.

When viewed against Campbell's frameworks of climate migration, the policy of 'migration with dignity' could be argued as more likely to address climate-induced migration, as it appears to indicate a level of choice around migration. Campbell defines climate-induced migration as a scenario in which the environment is adversely affected but not to the extent that continued human habitation becomes 
impossible (Campbell 2010). While migration with dignity allows for some to initiate a choice in when, how, and where to migrate, without an international policy that radically reduces emissions these choices will be eliminated.

The strategy of migration with dignity also indicates that forward planning will allow for pockets of Kiribati communities to become established in host countries, which, it is argued, will allow for cultural practices and identities to stay alive despite living away from Kiribati. The framing of migrating with dignity alludes to a level of agency in the process, particularly when we consider Tong's comments about IKiribati choosing host countries that they will migrate to and a belief that creating beachhead pockets of Kiribati communities will allow for I-Kiribati identity and culture to remain intact. However as a commentator for the Yale Globalist, Jessica Shor, argues,

There is one major difference, though, between the I-Kiribati already living abroad and those who will migrate under Tong's plan. Most I-Kiribati who now emigrate for work do so temporarily, sending remittances home and moving back to the islands after several years. In that way, they remain closely tied to their homeland. Yet under Tong's relocation plan, the I-Kiribati will abandon their homes forever. If the people of Kiribati must move abroad permanently, how long can they retain their cultural ties? And if the islands are submerged, to what homeland can IKiribati remain loyal? (Shor 2009: 1).

Shor's analysis of forced migration due to climate change points to the way that migration policies will impact on the culture of I-Kiribati. While Shor was but an undergraduate when this article was published, her comments sit within a wider academic discussion in which more advanced postgraduate-level researchers and academics have also questioned the role that migration has on cultural identity. Both Tabe and Teaiwa have illustrated the importance that land holds for I-Kiribati and have examined how migration has impacted on specific identities. Rajesh Chandra, writing in response to Hau'ofa's article 'Our Sea of Islands' also provides commentary around migration within the Pacific. Chandra notes that while migration has traditionally offered extended networks and access to resources, there are also negative consequences. Chandra argues, 
Migration, however, also deprives source areas of vital skills at critical times, and the final development outcome frequently depends on factors such as the rate of emigration and the replenishment of skills. Furthermore, links of emigrants with their homelands weaken over time. This is not to negate the very important point that Oceania is not limited to its physical space, either land or sea, but to simply indicate that emigration is problematic as a development strategy (Chandra 1993: 77).

Chandra's point is an important one to consider. Despite the fact that he is not writing with climate change in mind, the issues that he raises, such as cultural connection to land and also the depletion of on-island skills at a time of critical importance, are vital when thinking about the concept of migration with dignity and further brings into question the positive benefits of migration that Barnett and Campbell allude to.

Alofa presents a different strategy around migration to that presented by Tiimon. In an interview in 2010, when asked about migration she argues,

Me personally, talking about myself, in the long term we may leave, may leave Kiribati our country. What I cannot, what I am not prepared to accept, is that there is no preparation for my people. If we are moving we have to move at least to one place, to one country, one area, where we can continue to have our culture, we cannot just move in different countries we will lose everything (Alofa in 'Pelenise Alofa, President...' 9:57-10:29).

In this statement, Alofa proposes that, in order for culture to remain intact, I-Kiribati need to be relocated en masse to one location. This position is not only radically different to both Tiimon's and Tong's relocation proposals, but also offers a critique of their proposals. As has been presented in the section on the impacts of forced migration, Alofa has argued that for I-Kiribati, culture is intimately connected with having a space and land that is their own. Although migration with dignity may offer a degree of agency as there is the ability to make choices around when, how, and where to move, Alofa's call here places a stronger emphasis on I-Kiribati's need and ability to remain a sovereign nation, where their cultural traditions and identity can remain intact, albeit within a new geographical space. 


\section{Conclusion}

Both Alofa and Tiimon have articulated the position of the Pacific and specifically Kiribati in vastly different ways than the international community. Although they often use the language of the international community to describe Kiribati to the international community, for Tiimon and Alofa, Kiribati's vulnerability is talked about in a way that indicates that it is not an innate quality unique to the Pacific and islands within the Pacific, with climate change simply exacerbating this vulnerability, but rather that Kiribati is made vulnerable by climate change impacts that are largely not of Kiribati's doing. Although they refer to economic rationales for action or inaction, for them economic rationales are of less significance than arguments based on human rights.

These differing narratives and articulations of the impacts of climate change have led both Alofa and Tiimon to contextualise forced migration in ways that differ from the international community. The international commentary invokes images of 'sinking islands' and people fleeing from their homes as a result of the total degradation of the environment in the future. The images have been used to advocate for action to prevent this from happening. However, as has been argued, this articulation has allowed for the consequences of continued changes to climate to be viewed as something that does not require immediate action. In contrast both Alofa and Tiimon refer to the reality of rising sea levels and the impact that this is having in Kiribati now to argue for immediate action on climate change.

Tiimon and Alofa draw attention to that fact that climate induced migration is already happening. Despite this acknowledgment that climate change is already forcing people to migrate due to environmental degradation, they make it apparent that for them this does not need to continue nor become a situation where Kiribati is faced with total forced migration from a homeland that can never be returned to. By continuing to make demands of the international community to make immediate cuts in line with the scientific evidence, it is evident that both women still see a future for Kiribati. They nevertheless also make clear that if forced migration does become the final outcome for Kiribati, this will be due to the inaction of the international community.

It is with this in mind that Alofa and Tiimon have outlined the impact that such migration will have on their culture and identity. Both women have shown through their articulations of the impacts of forced migration that if their islands are lost, so 
too is their culture. This awareness of the consequences of climate change and the impact this will have on their culture has meant that both women have outlined the need for planning on how best to manage the total forced migration of Kiribati. What is interesting is the way these women have delivered vastly different concepts of how best to do this. While Tiimon has echoed the calls that are being made by President Tong to establish pockets of I-Kiribati in multiple national locations, Alofa has demanded that the bulk of Kiribati people be moved to one place- -thus affording a population concentration that, it is presumed, will be better able to keep their culture intact.

Although both Alofa and Tiimon are offering different ways for the Pacific and Kiribati to be viewed, the question remains: does their continued use of language that is so much like the language being used to describe them by the international community mean that their key points of divergence can be easily missed, dismissed, or ignored? And does this mean that, ultimately, forced migration will eventuate despite the calls from the Pacific demanding action? 


\section{Chapter Seven: Conclusion}

Vulnerability discourse runs right through climate change discussions-as a tool for categorising risk, an emotive term to encourage action, and appropriated and used to highlight responsibility. Vulnerable has become such an accepted and drawn-upon term that it is almost impossible to delve into the multitudinous climate change literature and not find references to vulnerability attached to women or Kiribati. As has been demonstrated, despite an attempt to bring clarity to how and why to assign such a term, there are varied and often conflicting interpretations being brought to bear on the use of the term in climate change discussions. This research has attempted to draw out the ways that vulnerability is being used, and examine how different actors within the climate change debate have brought different understandings and constructions into climate change discussions. These portrayals of vulnerability can be seen to not only impact the way that places and people are viewed but also to alter the way that solutions are talked about, particularly in regard to forced migration.

\section{Reviewing the Research Aims, Motivation and Approach}

The key question asked by this research has been how discourses of vulnerability have been applied within the context of the climate change debate and what impact this has on Kiribati, I-Kiribati women and forced migration. In order to answer this question there are several factors that have been explored, including who is using terms such as vulnerable, how they are being applied, and what the terms mean to those who are using them.

In order to understand the significant meaning that these terms are carrying, a qualitative approach, coupled with critical discourse analysis, has been used in an attempt to draw out the meaning that such terms are being invested with within the political context in which they are being used. For scholars such as Jennifer Brayton, it is qualitative research that allows for this type of meaning to be drawn out and analysed within a wider framework. When looking at the adjectives that have been used to ground vulnerability discourse in relation to the Pacific, it is clear that for those from outside the Pacific these terms have specific meanings and draw on rationales that are significantly different from the ideological underpinnings and meanings that are being invested by both Alofa and Tiimon. Drawing on critical discourse analysis further allows for the language being used to be assessed in relation 
to who is using the language, what meaning is attached to the language, and what impact the language has on the current climate change debate.

There has been significant debate and counter debate within feminist research on the impact that the use of terms such as vulnerable has on women. While this research has provided a theoretical basis useful for examining discourses of vulnerability, it fails to take into consideration the various differences between women. Rather, it presents arguments that position women dichotomously as either vulnerable or agents of change. This is a particularly important point to note when examining women within the climate change debate, as my research has argued this dichotomy is particularly blurred and a more nuanced approach is required. The use of the case study approach has allowed this research to pay particular attention to I-Kiribati women and examine how they are being spoken about and speaking about themselves. By doing this it has become clear that while the international community makes continued reference to women's vulnerability, and in some instances specifically I-Kiribati women's vulnerability, for both Alofa and Tiimon these articulations of vulnerability of women are much more complex than positioning women as intrinsically either vulnerable or agents of change.

\section{The Findings}

This research has demonstrated that vulnerability discourse has become an uncontested way of depicting particular places in regard to the risks they face due to climate change. While it has been acknowledged that such language has its place within the climate change debate, the continued and frequent use of such language has led to questions being asked about how this may impact on those who are being spoken about in this way. The approach taken in this research has allowed for the meanings that are being invested in terms such as vulnerable to be explored, drawing upon Pacific and feminist theory as a framework for analysis. What has become obvious throughout this research is that the ways in which vulnerability discourses have been invoked are plural and complex. This has required assessing the language that is being used from a wider perspective that takes into consideration broader historical and political understandings. In order to do this it has been imperative to examine the co-location of words that are being used to emphasise vulnerability and examine the differences between how Kiribati is being articulated by those from outside and those from within Kiribati. 


\section{Islands}

When we look at the ways in which Kiribati is being spoken about, we see that vulnerability discourse is not being used in isolation but rather is being anchored with the use of adjectives that are intended to ground the discourse within vulnerability frameworks. As was shown in Chapter three, terms such as small, isolated, developing etc, are all being used alongside vulnerability to indicate the level of vulnerability that Kiribati faces due to climate change. For scholars such as Nick Brooks these terms are viewed as a way of making explicit whether it is biophysical vulnerability or social vulnerability that is being spoken about. Based on the literature circulated by the IPCC it is apparent that Kiribati has been assessed and is viewed as vulnerable based on both its position as a low-lying island (biophysical vulnerability) and also its ability to respond to these threats (social vulnerability). While this research has not contested the science that indicates that climate change will have a disproportionate impact on places that are lower lying with little access to higher land, which Kiribati is, this research does contest the presumption that the adjectives that are being used to highlight this are based on climate change knowledge alone, rather than drawing on longer genealogies of framing the Pacific.

In examining the terms such as small, isolated, low-lying and developing, it has been shown that these terms are based more on Kiribati's economic position than its geographical dimensions. The categorisation of the Pacific in this way can be seen to have its roots in historical and political framings of the Pacific with views of the Pacific as small and isolated being produced from an economic and political centre outside of the Pacific. This long-held view of the Pacific as economically small and therefore vulnerable can be seen to have translated into how the Pacific is being viewed today within the climate change debate. While it is important to acknowledge the risks that islands within the Pacific face due to climate change, by constructing the image of the Pacific in this way, a view is created of places such as Kiribati as innately vulnerable. This expression of innate vulnerability has created a situation in which climate change can be viewed not as a pressure point creating vulnerability but instead merely highlighting pre-existing vulnerability inherent to Pacific Islands. This thesis contends that constructing Kiribati in this way allows for easier avoidance or dismissal of the politically and economically uncomfortable acknowledgment of responsibility of the international community in relation to mitigation and adaptation responses. Instead, the onus of responsibility is placed squarely on the shoulders of 
Kiribati. While this view of Kiribati persists, it creates a scenario where other constructions of Kiribati are unable to be explored, which this research contends has significant impacts on the way mitigation and adaptation strategies are being thought about.

Despite this framing of Kiribati in much of the climate change debate, it is clear that there are alternative ways that both the Pacific and Kiribati can and have been framed. Hau'ofa has been influential in challenging views of the Pacific; while outsiders may view the Pacific as small and isolated, he presents alternative views. He argues that the Pacific, rather than being small, is large when you consider the ocean, and that, rather than isolated, the islands within the Pacific are interconnected through trade, history, culture and genealogy. Hau'ofa contends that categorising the Pacific as small and isolated has established a belittling view of the Pacific that traps the Pacific into a cycle of dependence which is beneficial for those outside the Pacific. Hau'ofa's argument has subsequently been used by various other scholars who have questioned the way in which the Pacific is viewed and what impact this has on the policy approaches taken when working with the Pacific, either specifically when it comes to climate change (Barnett and Campbell 2010;) or more broadly (Fry 2010; Teaiwa, K 2005). Chapters four and five have used these alternative views of the Pacific, and critiques of the impacts that framing the Pacific in certain ways has, to examine how both Maria Tiimon and Pelenise Alofa have articulated Kiribati. The purpose of these chapters is to look at the way in which both these women are bringing conceptions of both the Pacific generally, and Kiribati specifically, into the climate change conversation. These chapters have questioned, first, whether they draw on a different articulation of Kiribati, and, secondly, how this then impacts on the way that they are characterising forced migration.

This thesis argues that while both Alofa and Tiimon may use the same language as the international community, the messages of vulnerability are being constructed in ways that indicate that-rather than having internalised conceptions of smallness and dependency, in line with Hau'ofa's critiques - they are using these constructions to fight the international community. Both Alofa and Tiimon have drawn on the fact that Pacific Islands are small to challenge the international community to take responsibility and act. This challenge sets Kiribati up as vulnerable in the sense that it has contributed little to the causes of climate change yet is going to be one of the first 
countries to feel the full impact, and this vulnerability, rather than being an innate quality, is a consequence of climate change.

This research contends that due to the complex nature of climate change, conceptions of Kiribati as innately vulnerability or conceptions of the Pacific as having agency and strength are limiting in their portrayal of the Pacific and Kiribati within the climate change debate. A parallel can be seen in the dichotomous characterisations of women as either vulnerable or agents of change, as discussed above. Hau'ofa presents the contrast between insider and outsider perspectives. This too, is nuanced, as Alofa and Tiimon speak of vulnerability but qualify it by underscoring the developed nations' responsibility for their actions that have created threats to Kiribati's existence. Alofa and Tiimon have drawn on vulnerability discourse as a way of both drawing attention to what is happening and demanding action from the international community. In doing so they have demonstrated the way in which Kiribati is vulnerable, based on its size, but more importantly, the fact that despite the fact that Kiribati has contributed very little to man-made climate change it is disproportionately bearing the brunt of impacts. They therefore present Kiribati's vulnerability as a created quality as opposed to an innate quality. This demonstrates the nuances that are present within the language that is being used by both those from outside the Pacific and those from within, and illustrates the importance of examining language with regard to the political context in which it is being used.

\section{Women}

The importance of having women included in climate change debate in order to increase gender sensitive policy approaches has been illustrated in both chapters two and four. While debate continues around how women should be depicted in order to have their needs meet, there is a consistency in the argument that, however women are portrayed, there is a very real need to for them to be included. Scholars have used gendered roles to highlight the ways in which climate change, generally, and forced migration, specifically, will have a disproportionate impact on women.

Chapter four drew on the concepts that were introduced in Chapter two regarding articulations of women and analysed them with particular focus on I-Kiribati women. Chapter four illustrated that both conceptions of women as vulnerable and conceptions of women as change agents are employed by those outside the region. Categorisation of women in these ways draws heavily on social vulnerability 
frameworks where social structures creating gendered roles are considered to be the driving force either exacerbating vulnerability or positioning women as agents of change. Looking at I-Kiribati women specifically, this thesis has shown that the women of Kiribati can be considered to be in a position of vulnerability according to social vulnerability frameworks. Chapter four highlighted their gendered roles and their limited involvement in decision-making to highlight this specific gendered vulnerability.

While social vulnerability frameworks have influenced the way that women are categorised, it has been shown that although gender may be the term that is being predominantly used, the focus is on 'women' as a single homogenous group. Chapter four made the argument that this categorisation of 'women' as a distinct group has turned discussions of gendered impacts of climate change into a discussion of how climate change affects 'women'. This thesis argues that this heavy reliance on sex as the categorising factor has seen all women positioned as vulnerable, which, even by omission, establishes all men as both the oppressor and having an equal strength and power within the climate change debate. I contend that framing the debate in this way does not allow for consideration of how gender, class, nationality, race and location are all intersecting factors that influence the differing levels of vulnerability that people face.

Despite constructions of women as vulnerable or women as agents of change appearing to sit in opposition with each other, Chapter four argues that both draw on essentialist notions of 'women'. While proponents of framing women as vulnerable (Dankelman 2011; Rohr 2011) have drawn on the social structures that influence the gendered roles that create vulnerability, those who argue for women to be seen as agents of change are drawing on similar frameworks of gender roles to make their case. Scholars in support of women being viewed as agents of change (Nayar 2011; MacGregor 2010; Murthy 2000) rather than seeing the gender roles of women as making them vulnerable, have repositioned the debate and argued that it is precisely because women have different gender roles that they are able to bring different and vital knowledge to the climate change debate and they should therefore be viewed as agents of change. In this attempt to reframe the way that women are viewed, these arguments are again drawing on a set notion of 'women' leaving little room for the complexities of how climate change is impacting on different groups. 
Chapter five examines Alofa's and Tiimon's commentary and finds that, while concern over why and how to include women in climate change discussions and policies may be of considerable importance for those outside of Kiribati, for these women the limited reference to women would indicate that it is not such a pressing issue for them. It appears that Tiimon has not referenced women at all. On the limited occasions that Alofa has, it has been in relation to their vulnerability. In both of Alofa's statements about women she has drawn on gendered roles such as mothering and food production to highlight why I-Kiribati women are particularly vulnerable to climate change.

Indigenous and black feminists have argued that, for women of colour, race is a more salient form of inequality than gender. These arguments have been introduced in Chapter five in order to consider explanations for the apparent limited conversation that is being produced about I-Kiribati women from Alofa and Tiimon. The myriad of complexities that have been brought into this research regarding understandings of women in the climate change debate has illustrated that there is no one definition of women that is helpful or applicable and the analysis in chapters four and five has served to further highlight this.

\section{Forced Migration}

The differing presentations of Kiribati and vulnerability have been shown throughout Chapters three, four and five to have influenced the way that forced migration is being discussed. While some scholars have continued to debate the definitional meanings of forced migration, there remains a fixation on the concept that in the future Kiribati will become so inundated by rising sea levels that I-Kiribati will be forced from their homes. Clearly, language use does not cause sea levels to rise, but language use shapes people's understanding of an issue as well as their concepts about how to address that issue. This research has asked, how have vulnerability discourses influenced the way that forced migration is being presented?

Chapter four contends that, coupled with the science, the representation of Kiribati as innately vulnerable based on its size and dependency has characterised forced migration as a realistic prospect and in some ways even presented it as an option that will benefit I-Kiribati. It is the depiction of Kiribati's innate vulnerability, it is argued, that has created a situation in which other strategies may have been overlooked or ignored. Looking at the IPCC's definitions of vulnerability and adaptation, the fact 
that Kiribati is considered vulnerable means that adaptation is an almost impossible task. Examining the alternative ways that both Alofa and Tiimon have presented Kiribati and articulated vulnerability demonstrates the significant impact that language plays in the presentation of forced migration. Both Alofa and Tiimon present forced migration as an outcome for Kiribati based on inaction by the international community, and vulnerability is expressed as a way of highlighting the unequal levels of responsibility for cause and response.

\section{Limitations of this Research}

This research has chosen to follow a case study approach. Making this choice has allowed for a greater scrutiny of the meanings that are being invested in particular language used to describe Kiribati and I-Kiribati women. While this thesis draws attention to the ways in which the Pacific has been spoken about broadly, the particular focus has remained on Kiribati as an island nation within the Pacific and on I-Kiribati women. As was noted in Chapter two, some Pacific studies scholars have critiqued the case study approach, citing its history as a way for researchers to test broad hypotheses while ignoring the differences between Pacific Islands (WesleySmith 1995). This is a critique that has been echoed by feminist researchers (Hill Collins 2004) who argue that this type of research ignores the differences that geographical space or position within society produces. Taking these critiques into consideration, it is extremely important to make explicit that this research is not intended to be used as a piece of work that can be applied homogenously throughout the Pacific. When examining the discourse of both Maria Tiimon and Pelenise Alofa, it is important to reiterate that this is an analysis of their words and is not intended to represent the voices of all I-Kiribati women.

The application of critical discourse analysis means that specific meanings have to be drawn out and then analysed with an awareness of the broader context in which they are being used. Throughout this thesis that has meant analysing language with an awareness of the broader political, historical and economic influences that are brought into the climate change debate with language such as vulnerable, one in which the power of language is analysed. However as Matheson has noted, this opens up the research to criticism around the meaning that the researcher chooses to apply based on the researcher's own politics. Taking this criticism on board, it is important to make this research as transparent as possible in relation to my own positionality. As a 
Pākeha woman from New Zealand I remain an outsider looking in. My understandings of meanings invested in language, while supplemented by the inclusion of Pacific theory, are generated by the position that I hold as an outsider. This became particularly obvious when examining the seeming lack of attention that both Alofa and Tiimon have paid to the particular issues of I-Kiribati women. While I have grounded my analysis in Pacific and black feminist theory in order to analyse this, I am still only able to make assumptions based on this theoretical knowledge. As a Pākeha woman my experience of the intersections of race and gender is from a position of the majority group in relation to race, which means that my experience of race and gender is from the perspective of the dominant race and the subordinate gender.

It is also important to note that in choosing to examine works by Alofa and Tiimon that already exist as part of the public record, as opposed to conducting interviews with each individual, the various contexts in which they are speaking has to be acknowledged. Both Alofa and Tiimon are delivering messages to the international community in what they see as the most effective way. As has already been demonstrated, the use of language by Alofa and Tiimon echoes language used to describe Kiribati by the international community. As the majority of Alofa's and Tiimon's interviews and speeches that have been used in this research are being directed at the international community, it is important to be mindful that what they are saying on the international stage is said for the purpose of delivering an effective message to encourage action. These messages are designed to attract attention and speak to those who can act on behalf of Kiribati, and may not provide the full depth or range of how they might articulate or view Kiribati, women, or forced migration in alternative contexts and amongst different audiences outside the public sphere.

In this same vein, it is important to consider the way that Alofa and Tiimon are being reported. Both women are delivering a message and the interests of those reporting can influence the way that this message is translated. In considering the lack of apparent attention that Alofa and Tiimon have paid to I-Kiribati women, it is important to question what influence those asking the questions of Alofa and Tiimon have on this. If this research had conducted first hand interviews of both Alofa and Tiimon, and asked specific questions about what climate change means to and for IKiribati women, it could potentially have generated articulations of women that have not been found in already reported interviews and messages. These questions arising 
from choices of how best to undertake this particular research leave room for further research on Kiribati, I-Kiribati women and forced migration.

\section{Conclusion}

Vulnerability is a value-laden term, with particular meaning attached by those who are using it, consequences for those who are being termed in this way, and impacts for the way responses to climate change are viewed. The innate vulnerability of types of places and types of people has been assumed by some and challenged by others, who have instead presented ideas of agency. Both constructions, however, manage to limit conceptions of islands and women into two contrasting boxes, assigning both women and islands innate qualities that make them either vulnerable or resilient. This research has argued that within the climate change context, viewing women or islands in either light does not allow for the complex challenges presented by climate change to be adequately explored.

When we look at constructions of island vulnerability, it is apparent that the international community is drawing on long-held views of the Pacific to reinforce vulnerability and at the same time downplay industrialised nations' own responsibility to act. This thesis contends that in the climate change debate, when vulnerability is attached to islands within the Pacific by the international community, climate change is treated as a spotlight on the innate vulnerability that islands, by their very nature, are thought to exhibit. This has not gone unchallenged. This thesis introduces theoretical arguments that provide a radically different view of the Pacific and demonstrate that there are other ways that the Pacific can be viewed that highlight agency and resiliency. However, neither conceptions of Pacific places such as Kiribati as either innately vulnerable nor as islands of strength and agency speak to the many and complex challenges posed by climate change. Both depictions potentially paint a simplistic picture of the Pacific and Kiribati.

Some of these complexities are illuminated when examining both Alofa's and Tiimon's articulations of Kiribati. Both Alofa and Tiimon refuse to adhere to either concepts of absolute vulnerability or absolute agency, instead they are forging a new path in which different constructions of Kiribati's vulnerability can be explored. Alofa and Tiimon draw on the vulnerability of Kiribati to demand action, yet show their strength by this very process of continuing to demand action. They use the language of the international community as a way of demonstrating the fact that they are 
victims of developed countries' consumption patterns, yet show their agency by defining their smallness in ways that that do not belittle them. They show their vulnerability to forced migration, however this weakness is reflected back to the international community as a weakness of the international community to act. The way that both Alofa and Tiimon are constructing Kiribati's vulnerability indicates that, within the climate change context, Kiribati is not innately vulnerable nor has it innate agency, and instead allows them to weave between the two conceptions. ${ }^{3}$

The analysis of women has illustrated that while constructions of women in the debate as either victims or agents of change are seemingly oppositional, both in fact are limiting in the way that they allow women to be considered. Alofa and Tiimon are living examples of the way that women can be both vulnerable and agents of change. Despite Alofa and Tiimon taking different approaches to the delivery of their messages-Alofa presents assertively and directly whereas Tiimon lets her emotions of sadness and fear show-both women are agents of change, as they are both standing up and demanding action for Kiribati and I-Kiribati people. However both women, in doing so, are making explicit their vulnerability with the language that they use.

Finally I will leave you with a quote from Alofa in which she demonstrates the way that vulnerability has been appropriated by her,

We need leaders who recognise the moral obligation to hear the voices of the most vulnerable... Real freedom is begot from within oneself. People can take away everything from us, but we will still remain free in our hearts and souls. My people will not give up fighting for justice, for our human right to life and a secure environment. We are calling loud and clear for climate justice NOW (Alofa 2009: 1).

\footnotetext{
${ }^{3}$ For related reading, see Teaiwa, K. (1999). Tirawata Irouia: Re-Presenting Banaban Histories. This MA work of Katerina M. Teaiwa discusses Banaban mobilisation of discourses of 'pity' related to the exploitation of Banaban land by British Phosphate Commission and the subsequent relocation of Banabans to Rabi island in Fiji. This work is in some ways similar to the project of this thesis in that it is an analysis of language viewed through historical and political contexts. However, Teaiwa's conclusions differ from this thesis's arguments about Alofa and Tiimon's mobilisation of discourse of vulnerability. Where Teaiwa argues that Banabans view themselves as to be pitied, this thesis contends that although Alofa and Tiimon use the term 'vulnerable', it is used to capture the world's attention, and that underneath these messages of vulnerability is a sense of strength and agency.
} 
This quote succinctly demonstrates the way Alofa and Tiimon use vulnerability, on their own terms, to show strength and resolve which could never be seen as small or dependent. 


\section{Bibliography}

Adger, N. (1999). Social Vulnerability to Climate Change and Extremes in Coastal Vietnam. World Development Vol. 27 (2), 249-269.

Adger, N \& Kelly, M. (2000). Theory and Practice in Assessing Vulnerability to Climate Change and Facilitating Adaptation. Climatic Change. 47; 325-352.

Alofa, P. (2:36). Pelenise Alofa at Pasifika Festival- Oxfam New Zealand. Retrieved May 29, 2012, from http://www.oxfam.org.nz/what-we-do/issues/climatechange/videos

Alofa, P (25:44). President of the Kiribati Climate Action Network at COP16- Online Video uploaded December 4, 2010, by OneWorldTV, accessed May 19, 2012, from http://www.youtube.com/watch?v=mZcNoMzr-jE

Alofa, P. (4:31).Pelenise Alofa talks about the human impact of climate change on her home in the Pacific islands- Online Video, Posted by Stacy King on December 8, 2010 from http://www.banabanvoice.ning.com/video/pelenise-alofa-talks-aboutthe

Alofa, P (2:56). One Hundred Days to Copenhagen- Online Video uploaded 23 August 2009 by OxfamNZ, accessed April 26, 2012 from http://www.youtube.com/watch?v=yq wHDTMyQA

Alofa, P. (25:45). Climate Action Network at COP16- Online Video uploaded December 4, 2010, by OneWorldTV, accessed May 19, 2012, from http://www.youtube.com/watch?v=mZcNoMzr-jE

Alofa, P. (2010). Climate Could Kill my Islands Culture. Poverty Matters Blog, theguardian Retrieved June 19, 2010 from http://www.guardian.co.uk/world/poverty-matters/2010/nov/09/kiribati-climatechange-voices-blog

Alofa, P. (3:08). Climate Testimonies: Pelenise Alofa, Kiribati- Online Video, uploaded 27 November 2009, By FriendsoftheEarthInt, accessed April 16, 2012 from http://www. Youtube.com/watch?v=JzyoENMWXtE

Alofa, P. (2011). Little or Small is Beautiful!!! A small island take on the stakes in Bonn. Retrieved May 2, 2012, from http://adoptanegotiator.org/author/pelenisealofa/ 
Alofa, P. (2011). My Banaban Experience. Posted by Montana Burgess CAN international August 15 2011, retrieved May 12, 2012, from http://www.climatenetwork.org/blog/event/1138

Alofa, P. (1:59). Paradise in Kiribati- Online Video, uploaded 11 December 2009 by OdysseyNetworks, accessed May 3, 2012, from http://www.youtube.com/watch?v=R-qYnJeFLHY\&feature=related

Alofa, P. (6:53). Voices of the Vulnerable- Online video, uploaded 8 November 2009 by OxfamNZ, accessed May19, 2012, from http://www.youtube.com/watch?v=x0sIMYqE3GY

Asian Development Bank. (2011). Climate Change and Migration in Asia and the Pacific. Retrieved April 5, 2011, from http://www.preventionweb.net/files/11673 climatechangemigration.pdf

Barnett, J. (2011). Forget that Sinking Feeling- there's nothing inevitable about climate change refugees. Retrieved September 4, 2011 from http://theconversation.edu.au/fprget-that-sinking-feeling-theres-nothing-inevitableabout-climate-change-refugees-814

Barnett, J. \& Adger, N. (2011). Climate Dangers and Atoll Countries. New Zealand; United Kingdom: Tyndall Centre for Climate Change Research.

Barnett, J. \& Campbell, J. (2010). Climate Change and Small Island States, Power, Knowledge, \& and the South Pacific. London; Washington, DC: Earthscan.

Barnett, J. \& Webbe, (2009). Accommodating Migration to Promote Adaptation to Climate Change: A Policy brief prepared for the Secretariat of the Swedish Commission on Climate Change and Development and the World Bank development report 2010 team, World Bank, Washington DC, and SCCCD, Stockholm.

Beckett, J. (1987). Torres Strait Islanders: Custom and Colonisation. Cambridge, Cambridge University Press.

Berg, B. (2009). Qualitative Research Methods for the Social Sciences. Boston: Pearson Education, Inc.

Black, R. (2001). Environmental Refugees: Myth or Reality? Working paper no34 University of Sussex, UNHCR Evaluation and Policy Analysis Unit Geneva.

Blomstrom, E. Cunningham, S. Johnson, N. Owren, C. (2009). Women at the Forefront. In Climate Change Connections. Retrieved September 9, 2011 from http://www.gender-climate.org/pdfs/ClimateChangeConnections.pdf 
Boano, C. Zetter, R. \& Morris, T. (2008). Environmentally Displaced People Understanding the Linkages Between Environmental Change, Livelihoods and Forced Migration. Refugee Studies Centre. Oxford: University of Oxford Bodansky, D. (2001). The History of the Global Climate Change Regime. In Urs Luterbacher \& Detlef F. Sprinz (Eds). International Relations and Global Climate Change. United States of America: Massachusetts Institute of Technology.

Bolin, B. Jager, J. \& Doos, B. (1986). The Greenhouse Effect, Climatic Change, and Ecosystems: A Synthesis of Present Knowledge. In Bert Bolin, Bo R. Doos, Jill Jager \& Richard A. Warrick (Eds) The Greenhouse Effect Climatic Change, and Ecosystems. Chichester. New York. Brisbane. Toronto. Singapore: John Willey \& Sons.

Boncour, P. \& Burson, B. (2009). Climate Change and Migration in the South Pacific Region: Policy Perspectives. Policy Quarterly, 5 (4), 13-20.

Brayton, J. (1997). What Makes Feminist Research Feminist? The Structure of Feminist Research. Retrieved October 18, 2011 from http://www.unb.ca/par1/win/feminmethod.htm

Briguglio, L. (1995). Small Island Developing States and their Economic Vulnerabilities. World Development Vol 23 (9) 1615-1632.

Brooks, N. (2003). Vulnerability, Risk and Adaptation: A Conceptual Framework. Tyndall Centre for Climate Change Research. Working Paper 38.

Brown, O. (2008). Human Development Report 2007/2008: Climate Change and Forced Migration: Observations, projections and implications. Retrieved August 30, 2011 from http://hdr.undp.org/en/reports/global/hdr20072008/papers/brown_oli.pdf

Brown, O. (2008). Migration and Climate Change. International Organization for Migration. Switzerland.

Brundtland, G. H. (1987). Address by Mrs Gro Harlem Brundtland at the Closing Ceremony of the Eighth and Final Meeting of the World Commission on Environment and Development. World Commission on Environment and Development. Retrieved July 28, 2012, from http://www.regjeringen.no/upload/SMK/vedlegg/

Burson, B (2010). Climate Change and Migration South Pacific Perspectives. Wellington: Victoria University. 
Butler, S. (2009). Pacific islanders struggle against climate change. In Green Left. Retrieved May 30, 2012, from http://www.greenleft.org.au/node/42103

Cameron, D. (1998). The Feminist Critique of Language: A Reader. London and New York: Routledge

Campbell, J. (2010). Climate Change and Population Movement in Pacific Island Countries. In Bruce Burson (eds). Climate Change and Migration South Pacific Perspective. Wellington: Victoria University.

Castels, S. (2002). Environmental change and forced migration: Making sense of the debate, working paper no 70. University of Oxford Refugee Studies Centre, UNHCR Evaluation and Policy Analysis Unit, Geneva.

Centre for Asia Pacific Women in Politics (2008). Third Global Congress of Women in Politics and Governance: Gender in Climate Change Adaptation and Disaster Risk Reduction. Retrieved August 30, 2011 from http://www.capwip.org/3rdglobalcongress1.html

Chandra, R. (1993). Where Do We Go From Here. In Eric Waddell, Vijay Naidu and Epeli Hau'ofa (Eds). A New Oceania: Rediscovering Our Sea of Islands. Suva: USP.

Climate Change in Kiribati (2010). Tarawa Climate Change Conference. Retrieved August 24, 2011 from http://www.climate.gov.ki/tarawa climate change conference.html

Climate Institute. (2011). Kiribati. Retrieved August 24, 2011 from http://www.climate.org/climatelab/Kiribati

Collins, D.N. (2009). Forced Migration and Resettlement in the Pacific: Development of a Model Addressing the Resettlement of Forced Migrants in the Pacific Islands Region from Analysis of the Banaban and Bikinian Cases. Retrieved February 7, 2013 from htpp://ir.canterbury.ac.nz/bitstream/10092/3234/1/thesis_fulltext.pdf

Collins, P. (2004). How Feminists Practice Social Research. In Sharlene Nagy HesseBiber \& Michelle L. Yaiser (Eds). Feminist Perspectives on Feminist Research. New York: Oxford University Press.

Cornwall, A. (2007). Revisiting the Gender Agenda. IDS Bulletin 38 (2) 69-78.

Creswell, J. (2009). Research Design: Qualitative, Quantitative, and Mixed Methods Approaches. United Kingdom: Sage Publications.

Cutter, S. (1996). Vulnerability to Environmental Hazards. Progress in Human Geography 20 (4), 529-539. 
Dankelman, I. (2011). Beyond Women and Girls’ Vulnerability: a debate on gender, climate change and disaster risk reduction. Retrieved September 4, 2011 from http://www.generoyambiente.org/arcangel2/documentos/530.pdf

Dankelman, I. (2009). Women: Agents of Change for a Healthy Environment. Retrieved July 17, 2012 from http://www.ww05.org/english/speech/5.2.ireneDankelman.pdf

Department of Energy and Climate Change. (2010). Cancun, Copenhagen, Kyoto and Rio. Retrieved 20 March, 2012, from http://www.decc.gov.uk

Denzin, N \& Lincoln, V. (2005). “Introduction” The Sage Handbook of Qualitative Research. Thousand Oaks, California: Sage Publications.

DeVault, M. (1996). Talking Back to Sociology: Distinctive Contributions of Feminist Methodology. Annual Review of Sociology. Vol 22 29-50.

Dessler, A. \& Parson, E. (2010). The Science and Politics of Global Climate Change: A Guide to the Debate. United Kingdom: Cambridge University Press.

Dubois, B. (1983). Passionate Scholarship: Notes on Values, Knowing, \& Method in Feminist Social Science. In Allen Rubin, Earl Babbie (Eds.) Research Methods for Social Work. United States of America: Brooks/Cole, Cengage Learning.

Dunbar, C. (2008). Critical Race Theory and Indigenous Methodologies. In Norman K. Denzin, Yvonna S. Lincoln \& Linda Tuhiwai Smith (Eds). Handbook of Critical and Indigenous Methodologies. United Kingdom: Sage Publications

Emberson, P. (2010). Statement of the Pacific Conference of Churches on Climate Change and Resettlement. On the Run: Europe and Pacific Responses to Climate Change in the Pacific. Germany. Pacific Networking in Europe. Retrieved September 2, 2011, from http://www.pacific-net.eu

European Commission. (2000), Push and Pull Factors of International Migration A Comparative Report. Luxembourg: Office for Official Publications of the European Communities.

Fairclough, N. Mulderring, J. \& Wodak, R. (1997). Critical Discourse Analysis. In Teun A. Van Dijk (Eds.) Discourse Studies: A Multidisciplinary Introduction. London: Sage Publications.

Farbotko, C. (2010). Wishful sinking: Disappearing islands, climate refugees and cosmopolitan experimentation. Asia Pacific Viewpoint, 51 (1), 47-60. 
Finnane, J. (2010). Pacific island Perspective On COP 15. In Aid Watch. Retrieved May16, 2012, from http://www.aidwatch.org.au/sites/aidwatch.org

Finucane, M. (2009). Why Science Alone Won’t Solve the Climate Crisis: Managing Climate Risks in the Pacific. Asia Pacific Issues. 89, 1-7.

Ford, J. (2008). Emerging Trends in Climate Change Policy: The Role of Adaptation. International Public Policy Review 3 (2), 5-15.

Fry, G. (1997). Framing the Islands: Knowledge \& Power in Changing Australian Images of the South Pacific. The Contemporary Pacific Vol 9 (2) pp 305-344.

Gemenne, F. (2011). Climate-Induced Population Displacement in a Four Degree plus World. Philosophical Transactions of The Royal Society 369, (182-195).

Governance and Social Development Resource Centre (2011). Understanding Vulnerability in the Context of Climate Change. Retrieved December 19, 2011 from http://www.gsdrc.org

Grey, S. (2006). Do Women Represent Women? Rethinking the “Critical Mass” Debate. Politics and Gender, 2 (4), 491-530.

Griffen, A. (1989). Defining Pacific Feminism. In Vanessa Griffen (Eds) Women, Development and Empowerment: A Pacific Feminist Perspective. Retrieved August 2, 2012 from http://www.nzetc. victoria.ac.nz/tm/scholarly/tei-GriWom2c5.html

Griffen, V. (2006). Gender Relations in Pacific Cultures and their Impact on the Growth and Development of children. Retrieved July 17, 2012 from http://www.unicef.org/eapro/Gender_Relations_In_Pacific_Cultures.pdf

Gutting, G. (2005). The Cambridge Companion to Foucault. New York: Cambridge University Press.

Haas, P. (2004). When does power listen to truth? A constructivist approach to the policy process. Journal of European Public Policy, vol 11 (4), pp 569-592.

Hagan, F. E. (2006). Research Methods in Criminal Justice and Criminology ( $7^{\text {th }}$ ed.). Boston: Allyn and Bacon.

Haigh, C. \& Vallely, B. (2010). Gender and the Climate Agenda: The impacts of Climate Change on Women and Public Policy. Retrieved April 5, 2011, from http://www.wen.org.uk

Haraway, D. (1998). Situated Knowledges: The Science Question in Feminism and the Privilege of Partial Perspective. Feminist Studies, Vol 14 (3), pp575-599. 
Haraway, D. (1991). Simians, Cyborgs, and Women: The Reinvention of Nature. United States of America: Routledge

Hau'ofa, E. (2008). The Ocean in Us. We are the Ocean: Selected Works. Honolulu: University of Hawaii Press.

Hau'ofa, E. (2008). “Our Sea of Islands.” We are the Ocean: Selected Works. Honolulu: University of Hawaii Press.

Hay, J. Mimura, N. Campbell, . Fifita, S. Kosy, K. McLean, R. Nakalevu, T. Nunn, P. \& Wet, N. (2011). Climate Variability and Change and Sea Level Rise in the Pacific Island Region: A Resource Book for Policy and Decision Makers, Educators and other Stakeholders. Retrieved August 24, 2011 from http://research.fit.edu/sealevelriselibrary/documents/doc mgr/334/Pacific Islands SLR Climate Variability Resource Book - Hay et al.pdf

Hemmati, M \& Rohr, U. (2009). Engendering the Climate - Change Negotiations: Experiences, Challenges, and Steps Forward. Gender and Development 17 (1) 1932.

Hill Collins, P (2000). Black Feminist Thought: Knowledge, Consciousness, and the Politics of Empowerment. New York; London: Routledge.

Holan-Hako, H. (2011). Why is gender an important consideration in the context of adapting to climate change and disaster risk management in the Pacific? Retrieved September 4, 2011 from http://www.capwip.org/closing remarks holan CAPWIP Pacific.pdf

Houghton, J. (2002). An Overview of the Intergovernmental Panel on Climate Change (IPCC) and its Process of Science Assessment. Issues in Environmental Science and Technology, No 17. Global Environmental Change.

Huffer, E \& Qalo, R. (2004). Have We Been Thinking Upside-Down? The Contemporary Emergence of Pacific Theoretical Thought. The Contemporary Pacific. Spring, 89-116.

International Institute for Sustainable Development in Cooperation with UNDP, UNEP, FAO, UNFCCC (2009). Oxfam international climate hearing at Copenhagen. Retrieved May 28, 2012, from http://www.iisd.ca/climate/COP15/enbots/ Intergovernmental Panel on Climate Change (IPCC). (2011). History. Retrieved July 30, 2011; from http://www.ipcc.ch/organization/organization history.shtml 
Intergovernmental Panel on Climate Change (IPCC). (2007). Fourth Assessment Report: Impacts, Adaptation and Vulnerability. Retrieved July 30, 2011 from http://www.ipcc.ch/publications_and_data/publications/publications_and_reports.s $\underline{\text { html }}$

Intergovernmental Panel on Climate Change (IPCC). (1990). Impacts, Adaptation and Vulnerability. Retrieved July 30, 2011 from http://www.ipcc.ch/publications and data/publications/publications and reports.s $\underline{\text { html }}$

Ireland, P. (2010). Fighting for Survival in Cancun. Retrieved April 10, 2012, from http://aclimateforchange.org/profiles/blogs/fighting-for-survival-in

ISIS International. (2012). Gender and Climate Change: Toolkit for Women on Climate Change. Retrieved July 29, 2012 from http://www.isiswomen.org/index Jackson, (1993). Doing What Comes Natutrally? Women and Environment in Development. World Development. 21 (12) 1947-1963.

Jumeau, R. (2009). Many Strong Voices-turning vulnerability into strength. Retrieved September 4, 2011 from http://www.manystrongvoices.org

Kandlikar, M \& Sagar, A. (1999). Climate change research and analysis in India: an integrated assessment of a south-north divide. Global Environmental Change, 9, 119-138.

Kempf, W. (2008). A Sea of Environmental Refugees? Oceania in an Age of Climate Change. Retrieved June 9, 2011, from http://www.unigoettingen.de/de/document

Kidu, C. (2011). Call for Gender Integration into Climate Change Agenda. Retrieved August 28, 2011 from http://pacific.scoop.co.nz/2011/07/callfor-gender-intergration-into-cliamte-change-agenda/

Kiribati Government. (2011). Climate Change in Kiribati: The climate change portal of the Office of the President of Kiribati. Retrieved August 20, 2011 from http://www.climate.gov.ki/climate change effects in kiribati.html

Kiribati National Statistics Office. (2005). Social Statistics, Demographic IndicatorsKey Demographic Indicators from Census of Population and Housing. Retrieved July 29, 2012 from http://www.spc.int/country/KI/stats/

Lane, R. \& McNaught, R. (2009). Building Gendered Approaches to Adaptation in the Pacific. Retrieved June 15, 2012 from 
http://www.pacificdisaster.net/pdnadmin/data/original/oxfam_2009_gendered_app roaches pacific.pdf

Liverman, D. (1990). Vulnerability to Global Change. In R. Kasperson (ed). Understanding Global Environmnetal Change: The Contributions of Risk Analysis and Manangment. Report on an International Workshop, Clark University, Earth Transformed Program: Worcester MA.

Locke, J. (2009). Climate Change-Induced Migration in the Pacific Region: Sudden Crisis and Long-Term Developments. The Geographical Journal 175 (3), pp.171180.

Loughry, M. \& McAdam, J. (2008). Kiribati- Relocation and Adaptation. Forced Migration review: Climate Change and Displacement. Retrieved April 5, 2011, from http://www.forcedmigration.org/policy

McEwan, C. (2001). Postcolonialism, Feminism and Development: Intersections and Dilemmas. Progress in Development Studies 1 (2), pp. 93-111.

MacGregor, S. (2010). Gender and Climate Change: From Impacts to Discourses. Journal of the Indian Ocean Region Vol 6 (2) pp.223-238.

Maclellan, N. (2010). Environment: Mixed Response to Cancun Meeting But Still more to be done by the Pacific. Islands Business. Retrieved 17 March, 2010 from http://wwwislandbusiness.com

Manion, H.K. (1995). Ecofeminism Within Gender and Development. Retrieved July 17, 2012 from http://www.ecofem.org/jounal

Matheson, (2008). Critiquing the Critical: A Reflection on Critical Discourse Analysis. Communications Journal of New Zealand 9 (1), pp.83-96.

Mauthner, N. \& Doucet, A. (1998). Reflections on Voice -centred Relational Method: Analysing Maternal and Domestic Voices. Ribbens, J \& Edwards, R. (Eds). Feminist Dilemmas in Qualitative Research: Public Knowledge and Private Lives. London. Sage Publications.

Maykut, P \& Morehouse, R (1994). Beginning Qualitative Researchers: A Philosophical \& Practical Guide. Washington DC: Falmer.

Maynard, M. (1994). Methods, Practice and Epistemology: The Debate about Feminism and Research. M, Maynard \& J, Puruis. (Eds.) Researching Women's Lives from a Feminist Perspective. United Kingdom. Burgess Science Press. 
Mensah-Kutin, R. (2010). Gender and Climate Change Issues: The Challenges for Policy Advocacy in Ghana. Retrieved June 9, 2011, from http://www.g-rap.org/docs/gender/gender_forum

Mercer, P. (2009). World Bank issues bleak outlook for Pacific islands nations. Retrieved May 5, 2012, from http://www.thenational.ae/news/world/asiapacific/world-bank-issues-bleak-outlook-for-pacific-islands-nations

Murthy, R. (2000). Disaster Legislations in Six Asia-Pacific Countries: A review from an SRHR \& Feminist Lens. ARROWs for Change, 14 (3), 2008, 11-12. Retrieved June 23, 2011, from http://www.arrow.org.my/images/publications

Nayar, A. (2011). Countering False Solutions \& Population Stabilization Arguments. Association for Women’s Rights in Development. Retrieved June 9, 2011, from http://www.awid.org/library/new-resources-articles

Ngaebi, T. Russell, T. \& Tamuera, F. (1993). The Status of Women. In Howard Van Trease (Ed) Atoll Policics: The Republic of Kiribati. Macmillan Brown Centre for Pacific Studies and Institute of Pacific Studies: New Zealand.

Nightingale, A. (2006).

Office of the President of Kiribati. (2011). Climate Change in Kiribati. Retrieved May16, 2011 from http://www.climate.gov.ki

Oxfam (2009). Suffering the Science: Climate Change, People and Poverty. Retrieved 17 March, 2012 from http://www.oxfam.org/sites/www.oxfam.org/files/bp130suffering-the-science.pdf

Parikh, J. (2009). Toward A Gender-Sensitive Agenda For Energy, Environment And Climate Change. Division for the Advancement of Women. Department of Economic and Social Affairs. United Nations. Retrieved September 4, 2011 from http://www.un.org/womenwatch/daw

Park, S. (2009). Climate Change and the Risk of Statelessness: The Situation of LowLying Island States. Legal and Protection Policy Research Series. Retrieved September 1, 2011 from http://www.unhcr.org/4df9cb0c9.html

Paterson, M. (1996). Global Warming and Global Politics. London: Routledge.

Patton, M. (1999). Enhancing Quality and Credibility. Health service research 34:5 part 2 (December 1999). Retrieved November 11, 2011 from http://www.ncib.nlm.nih.gov/pmc/articles.../hsresearch0002-0112.pdf 
Pelling, M \& Uitto, J. (2001). Small Island Developing States: Natural Disaster Vulnerability and Global Change. Environmental Hazards, 3, 49-62.

Pettenger, M. (2007). The Social Construction of Climate Change: Power, Knowledge, Norms, Discourses. England: Ashgate Publishing.

Porsanger, J. (2004). An Essay about Indigenous Methodology. Retrieved August 9, 2012 from http://www.septentrio.uit.no/index.php/nordlit/article/view/1910/1776

Ramazanoglu, C. \& Holland, J. (2002). Researching 'others': Feminist methodology and the politics of difference. In Ramazanoglu, C. \& Holland, J. (Eds) Feminist Methodology Challenges and Choices. London. Sage Publications.

Refugee Studies Centre. (2008). Forced Migration Research and Policy Overview. Retrieved April 5, 2011, from http://www.forcedmigration.org/policy

Resurreccion, B. (2001). The Gender and Climate Debate: More of the Same or New Pathways of Thinking and Doing? Asia Security Initiative Policy Series No 10, Singapore: RSIS Centre for Non- Traditional Security (NTS) Studies.

Riles, Annelise. (2001). The Network Inside Out. Ann Arbor: University of Michigan.

Risse, M. (2009). The Right to Relocation: Disappearing Island Nations and Common Ownership of the Earth. Larnegie Council for Ethics. Retrieved August 4, 2012 from http://www.hks.havard.edu

Rohr, U. (2011). Beyond Women and Girls’ Vulnerability: a debate on gender, climate change and disaster risk reduction. Retrieved September 4, 2011 from http://www.generoyambiente.org/arcangel2/documentos/530.pdf

Runci, P. (2007). Expanding the participation of developing country scientists in international climate change research, Environmental Practice, vol 9 (4), pp225227.

Sardar, Z. (1999). Development and the Locations of Eurocentrism. In Ronald Murick and Denis O Hearn (eds). Critical Development Theory-Contributions to a New Paradigm. London: Zed Books

Schipper, L. (2007). Climate Change Adaptation and Development: Exploring the Linkages. United Kingdom: Tyndall Centre for Climate Change Research.

Shor, J. (2009). The Tides of Change. In The Yale Globalist. Retrieved May 30, 2012, from http://www.tyglobalist.org/front-page/theme/the-tides-of-change/

Stake, R. (1995). The art of the case study research. United States of America: Sage Publications. 
Stake, R. (1994). Case Studies. In N.K. Denzin \& Y.S. Lincoln (Eds.), Handbook of Qualitative Research. Thousand Oaks, CA: Sage.

Steiner, A. (2008). Forced Migration Review: Climate Change and Displacement. Retrieved August 30, 2011 from http://www.fmreview.org/FMRpdfs/FMR31/FMR.31.pdf

Sullivan, R (2012). Arohanui. Paula Green (Ed). In Dear Heart 150 New Zealand Love Poems. New Zealand: Random House.

Tabe, T. (2011). Sapon Riki Ba Kain Toromon: A Study of the I-Kiribati Community in Solomon Islands. Retrieved February 20, 2013 from https://scholarspace.manoa.hawaii.edu/.../Tabe_2011_r.pdf

Teaiwa, K. (2005). Our Sea of Phosphate: The Diaspora of Ocean Island. In G. A. Harvey \& C. D. Thompson (Ed.), Indigenous Diasporas and Dislocations: Unsettling Western Fixations. England: Ashgate Publishing Limited.

Teaiwa, K. (1999). Tirawata Irouia: Re-Presenting Banaban Histories. Retrieved February 20, 2013 from https://scholarspace.manoa.hawaii.edu/

Tegart, W. Sheldon, G.W. \& Griffiths, D.C. (1990). The IPCC Impact Assessment Report. Retrieved January 5, 2012 from http://www.ipcc.ch/publications and data/publications ipcc first assessment 199 $\underline{0}$ wg2.shtml

Tellis, (1997). Application of a Case Study. The Qualitative Report, Vol 3 (3) retrieved April 24, 2012 from http://www.nova.edu/ssss/QR/QR3 3/tellis2.html

Thaysen, C. (2009). Pacific Islanders Call for Help (Banaban Pelenise Alofa). Posted by Stacy King December 9, 2009, accessed May 16, 2012, from http://banabanvoice.ning.com/profiles/blogs/pacific-islanders-call-out-for

The Women’s Environment Network (2010). Gender and the Climate Change Agenda: The Impacts of Climate Change on Women and Public Policy. Retrieved April 24, 2012 from http://www.wen.org.uk/general/gender-and-the-climatechange-agenda

The World Bank (2011). Gender and Climate Change: Three Things you Should Know. Retrieved April 24, 2012 from http://web.worldbank.org Tiimon, M. (5:52). Climate Change: Pacific Calling- Online Video, uploaded 8 September, 2009, by AusConservationTV, accessed April 27, 2012, from http://www.youtube.com/watch?v=Jb1Xa1AMjqY 
Tiimon, M. (2011). Human Faces and Stories from Kiribati. Retrieved May 19, 2012, from http://www.sideshare.net

Tiimon, M. (2009). Pacific Calling Partnership. In What Pacific Calling Partnership did at COP15 Copenhagen: Pacific Islanders and Australians Partnering Together in Advocating on the Human Face of Climate Change. Retrieved May 16, 2012, from http://www.erc.org.au/index.php?module=documents\&JAS

Tiimon, M. (2011). Pacific island and climate change: the situation of Kiribati. On The Run: European and Pacific Responses to Climate Change in the Pacific. Retrieved June 16, 2012 from http://www.pacific$\underline{\text { net.eu/dnn/linkclick.aspx }}$

Tiimon, M. (2:12). Sinking Paradise- Online Video, uploaded 21 July, 2009, Retrieved May 17, 2012, from http://www.foe.co.uk/news/sinking_paradise 20803.html

Tiimon, M. (2010). Speech by Maria Tiimon at Sydney Palm Sunday 2010. Sydney Peace and Justice Coalition. Retrieved April 15, 2012, from http://www.nswpeace.org.au/features/1270788116_6611.html

Tiimon, M. (2011). The Human Face of Climate Change. Retrieved June 16, 2012 from http://www.compassreview.org/spring11/5.pdf

Timmerman, P. (1981). Vulnerability, Resilience and the Collapse of

Society: a review of models and possible climatic applications.

Canada: Institute for Environmental Studies.

Tong, A. (2012). Relocating from climate change. In Climate change in Kiribati: The climate change portal of the Office of the President of Kiribati. Retrieved June 6, 2012, from http://www.climate.gov.ki/kiribati_climate_change_strategies.html

Trask, H.K. (1993). From a Native Daughter. Monroe: Common Courage Press.

Tuhiwai-Smith, L. (1999). Decolonizing Methodologies: Research and Indigenous Peoples. London: Zed Books.

UN WomenWatch. Fact Sheet: Women, Gender Equality and Climate Change. Retrieved December 16, 2011 from http://www.un.org/womenwatch

UNESCO (2009). Country Programming Document: Kiribati 2008- 2013. Retrieved May 16, 2012 from http://unesdoc.unesco.org/images/0018/001832/183241e.pdf United Nations Development Programme (2009). Resource Guide on Gender and Climate Change. Retrieved April 24, 2012 from http://www.un.org/womenwatch/downloads/resource Guide English FINAL.pdf 
Van Dijk, T. (1998). Critical Discourse Analysis. Retrieved November 11, 2011 from http://www.hum.uva.nl/ teun/cda.htm

Vine, D. (2009). Island of Shame: The Secret History of the U.S. Military Base on Diego Garcia. United States of America: Princeton University Press.

Waddell, E. Naidu, V \& Hau'ofa, E. (1993). A New Oceania: Rediscovering Our Sea of Islands. Suva: USP.

Warrick, O. (2011). Local Voices, Local Choices? Vulnerability to Climate Change and the Community-Based Adaptation in Rural Vanuatu. New Zealand: University of Waikato.

Wesley-Smith, T. (1995). Rethinking Pacific Studies. Pacific Studies vol 18 (2), 115137.

Weart, S. (2003). The Discovery of Global Warming. London, England. Harvard University Press.

Westmarland, N. (2001). The Quantitative/Qualitative Debate and Feminist Research: A Subjective View of Objectivity. Retrieved August 17, 2011, from http://www.qualitative-research.net

Wood, H. (2003). Cultural Studies for Oceania. The Contemporary Pacific 15 (2), 340-374.

World Meteorological Organisation (1986). Report of the International Conference on the Assessment of the role of carbon dioxide and of other greenhouse gases in climate variations and associated impacts, Villiach Austria. Retrieved July 28, 2012 from http://www.icsu-scope.org/downloadpubs/scope29/statement.html

Yin, R. (1984). Case Study Research: Design and Methods. Newbury Park, CA: Sage Publications. 